Meddelanden frän Stockholms Högskolas Mineralogiska Institut

Nir 51. 


\title{
RÖNTGENOGRAPHISCHE UNTERSUCHUNG DER KRISTALLSTRUKTUREN VON MAGNETKIES BREITHAUP'TIT, PENTLANDIT, MILLERIT UND VERWANDTEN VERBINDUNGEN
}

\author{
INAUGURAL-DISSERTATION \\ ZUR
}

ERLANGUNG DER DOKTORWÜRDE

MIT

GENEHMIGUNG DER MATH.-NATURWISSENSCHAFTLICHEN

FAKULTÄT DER HOCHSCHULE ZU STOCKHOLM ZUR ÖFFENTLICHEN

BEURTEILUNG VORGELEGT

VON

$N I L S A L S E ́ N$

DIE VERTEIDIGUNG VIRD AM 21 MÄR'TZ 1925,10 UHR VORMITTAGS, IM HÖRSAALE N:R 1 STATTFINDEN

STOCKHOLM 1925

KUNGL. BOKTRYCKERIET. P. A. NORSTEDT \& SÖNER

250333 

Röntgenographische Untersuchung der Kristallstrukturen von Magnetkies, Breithauptit, Pentlandit, Millerit und verwandten Verbindungen.

\author{
Von \\ Nilis Alsén.
}

(Hierzu Tafel 1-4.)

\title{
INHALTSVERZEICHNIS.
}

Einleitung $\cdot \cdot \cdot \cdot 0$

Apparatur . . . . . . . . . . . . . . . . . . . . 23

Material . . . . . . . . . . . . . . . . . . 23

Magnetkies . . . . . . . . . . . . . . . . . 26

Breithauptit . . . . . . . . . . . . . . . . . . . . . . . 39

Rotnickelkies . . . . . . . . . . . . . . . . . . . . .45

Künstlich dargestellte Verbindungen mit derselben Struktur wie Magnetkies . . . . . . . . . . . . . . . . . . . . . . . 47

Gültigkeitsbereich der Kristallstruktur Typus Rotnickelkies . . . . . 53

Pentlandit . . . . . . . . . . . . . . . . . 54

Millerit . . . . . . . . . . . . . . . . . . . . . . 62

Zusammenfassung . . . . . . . . . . . . . . . . . . . . . 71

\section{Einleitung.}

Als Verf. sich vor ein paar Jahren auf Veranlassung von D:r G. AmrNoff zu der vorliegenden Untersuchung entschloss, bezweckte er mit derselben, zwei Probleme auf röntgenographischem Wege zu lösen. Das eine war die alte Frage von dem Schwefelüberschuss des Magnetkieses über die Formel FeS hinaus, das zweite betraf den gegenseitigen Zusammenhang zwischen den NiS-Mineralien Millerit und Beyrichit und deren Relation zum Magnetkies, der ja oft mehrere Prozent Nickel enthält. 
Die älteste quantitative Analyse von Magnetkies aus dem Jahre 1804 ergab als Resultat die Formel FeS exakt, aber es dauerte nicht viele Jahre, bis der Schwefelüberschuss konstatiert wurde, und seitdem ist das Magnetkiesproblem dauernd aktuell gewesen. Hier sollen nicht die vielen Formelvorschläge referiert werden, nur als Kuriosum sei folgendes aus einer Abhandlung von BERzeLIUS $^{1}$ angeführt. „Wenn man bei Bereitung von Schwefeleisen das Eisen im Überschusse zusetzt, wird während des Schmelzens ein Anteil metallisches Eisens in dem Schwefeleisen aufgelöst, und diese Auflösung kann eben solche unmerkliche Abstufungen haben, wie z. B. die Auflösung eines Salzes in Wasser.»

Nicht einmal der Fund Brezinas ${ }^{2}$ in einem Drusenraume eines sonst compacten Meteoreisens von einem Troilitkristall, der Formengleichbeit mit Magnetkies zeigte, konnte eine einstimmige Meinung in dieser Frage hervorbringen.

Auch in den letzten Jahrzehnten wurde die Diskussion fortgeführt. So haben die Amerikaner Aluen, Crenshaw und J. JohnssoN in einem Aufsatz $\triangleq$ On the mineral sulphides of iron ${ }^{3}$ konstatiert, dass die wechselnde Zusammensetzung synthetisch dargestellter Magnetkiese von der Temperatur und dem Druck des Schwefeldampfes abhängig ist, in welchem sie erhitzt worden sind. Die Verff. kommen zu dem Resultat, dass, da das spez. Gewicht sich mit dem Schwefelgehalt kontinuierlich verändert, Magnetkies eine feste Lösung von Schwefel in FeS, und Troilit nur das Schlussglied der Magnetkiesserie ist. Gegen diese Auffassung hat ein anderer Amerikaner, EAKLE, ${ }^{4}$ bestimmten Einspruch erhoben in einem Werk über tellurisches Troilit aus Del Norte County, California. E. konstatiert, dass Troilit sich in drei Hinsichten von Magnetkies unterscheidet:

a) es hat eine etwas andere Farbe,

b) ist nicht magnetisch und

c) ist leicht löslich in verdünnter Schwefelsäure.

EAKLE meint, dass die Unterschiede zwischen den beiden Mineralien so wesentlich sind, dass der winzige Zuschuss von $\mathbf{S}$ in fester Lösung zu FeS die spezifischen Eigenschaften des Magnetkieses nicht erklären kann.

Statt dessen wirft E. die Vermutung auf, der Magnetkies bilde eine feste Lösung von $\mathrm{FeS}$ in $\mathrm{Fe}_{3} \mathrm{~S}_{4}$; die letztere hypothetische

i Ostwalds Klassiker 35, 34.

2 Sitzb. Ak. Wien 1881, 83, 473.

3 Am. Journal of Science. XXXIII, 1912, 169.

4 The Am. Mineralogist 1922, 7, 77. 
Verbindung sollte auf Grund ihrer Ähnlichkeit mit $\mathrm{Fe}_{3} \mathrm{O}_{4}$ magnetisch sein.

Die seitherigen Resultate der vorliegenden Untersuchung scheinen die Auffassung von Allen, Crenshaw und J. Johnsson zu bestätigen.

Dass die beiden NiS-Mineralien, Millerit und Beyrichit, trotz vollständiger kristallographischer Übereinstimmung, verschiedene Mineralien sind, scheint recht unwahrscheinlich, wenn auch Farbe und spez. Gewicht etwas verschieden sind. Es schien daher von einem gewissen Interesse, sie einer röntgenographischen Untersuchung zu unterziehen, bei welcher ein eventueller Untersthied zwischen denselben natürlich verschiedene Röntgenogramme veranlassen würde. Bei dieser Untersuchung hat das eine als Beyrichit etikettierte Material mit dem Millerit identische Photogramme gegeben. Aus dem anderen wurde ein nicht hexagonales Lauephotogramm erhalten und deswegen wurde eine qualitative Analyse der betreffenden Substanz vorgenommen. Hierbei stellte sich heraus, dass sie weder Nickel noch Schwefel wohl aber Phosphor und Eisen enthielt.

Trotz energischer Versuche, wobei Prof. Belowskr in Berlin keine Mühe gescheut hat, mir behilflich zu sein, ist es bisher nicht gelungen, auch nur das geringste Quantum von den Beyrichitoriginalen zu erhalten. Unter solchen Umständen wäre es zu früh, das Beyrichit definitiv als selbständiges Mineral auszusondern, wenn auch vieles dafür spricht. Für Millerit ist indessen eine wahrscheinliche Kristallstruktur hergeleitet worden.

Die Magnetkiese weisen häufig grossen Nickelgehalt auf, ohne dass die Kristallstruktur sich ändert. Es schien Verf. darum von grossem Interesse zu sehen, ob der Fe-Gehalt bis zu null vermindert werden konnte. Verf. hat darum von NiS, das durch Synthese dargestellt ist, Pulverphotogramme aufgenommen und völlige Analogie mit denen des Magnetkieses konstatieren können. Verf. hat auch gefunden, dass sowohl FeS als auch NiS sich in gleicher Weise darstellen lassen, nämlich durch Erhitzung in Schwefelwasserstoffatmosphäre von gefälltem Sulfid, was von gewissem geologischen Interesse sein dürfte.

Der bei Magnetkies und Nickelmagnetkies gefundene Strukturtyp ist derselbe, den AMINOFF ${ }^{1}$ bei Rotnickelkies konstatiert hat. Dadurch gesellte sich zu den anderen Problemen noch dieses: welches Gültigkeitsgebiet besitzt dieser Strukturtyp? Aus diesem Grunde wurden die Verbindungen FeSe, FeTe, NiSe, NiSb und CoS

1 Z. Krist. LVIII. 1923, 203. 
untersucht, von NiSb auch das Mineral Breithauptit. Es hat sich gezeigt, dass alle bis auf $\mathrm{FeTe}$, für das die Untersuchung bisher negatives Resultat gegeben hat, denselben Strukturtyp wie FeS darstellen. Nebenbei ist eine röntgenographische Untersuchung von Covellin (CuS) von Verf. angefangen und es ist schon klar, dass Covellin eine, andere Kristallstruktur als Magnetkies haben muss.

Schliesslich schien es angezeigt, die Kristallstruktur des Pentlandits (Fe, Ni)S klarzustellen, die von Groth ${ }^{1}$ als ein Beweis für das Vorhandensein kubischer Modifikationen von sowohl NiS als auch FeS angesehen wird. Doch stellt auch G. die Möglichkeit in Frage, dass Pentlandit eine wirkliche chemische Verbindung ist. Die Untersuchung des Verf. hat dies als sicher nachgewiesen, indem die Photogramme von P. offenbar nur einer einzigen Verbindung angehören.

Das Untersuchungsmaterial von natürlichen Kristallen wurde in erster Linie von dem mineralogischen Institut der Stockholmer Hochschule durch prof. P. Quensel zur Verfügung gestellt, weiter von der mineralogischen Abteilung des Reichsmuseums durch Prof. G. Aminofe und D:r N. Zenzén, vom Metallographischen Institut durch Prof. C. Benedicks und endlich von der Technischen Hochschule in Trondhjem durch Prof. J. H. L. Vogr. Den genannten Herren möchte ich an dieser Stelle meinen herzlichen Dank aussprechen.

Den grössten Dank schulde ich meinem Lehrer in der Röntgenkristallographie, Prof. G. Aminoff, der mich in diesen neuen Wissenschaftszweig einführte. Ausserdem danke ich ihm für die Beihülfe, die er mir seither mit nie versagender Bereitwilligkeit im Verlauf der Arbeit gewährt hat. Auch dem Direktor des mineralogischen Instituts Prof. P. Quensel, der mir stets das lebhafteste Entgegenkommen gezeigt, und meine Árbeit auf verschiedene Art gefördert hat, bin ich zu grösstem Dank verpflichtet. Den Kollegen im Röntgeninstitut A. Westaren und G. Phragmén möchte ich vor allem danken für die weitherzige Bereitwilligkeit, mich die von ihnen konstruierte Apparate benutzen zu lassen, was mir meine Arbeit im höchsten Grade erleichtert hat.

Für pekuniäre Unterstützung möchte ich der Königl. Akademie der Wissenschaften, die mir im vorigen Jahre ihr Beskowsches Stipendium zuerteilte, meine Dankbarkeit bezeugen.

1 Chemische Krystallographie I, 138 


\section{Apparatur.}

Die Stockholmer Hochschule verfügt über Röntgenapparaturen zur Aufnahme von Lauephotogrammen und Pulver- und Drehphotogrammen. ${ }^{1}$ In ersterem wird die sweisse» Strahlung von einem Lilienfeldrohr mit ungefähr 80 Kilovolt Spannung und 8 Milliampère Stromstärke erhalten. Die monochromatische Strahlung wird von einer Fe-Antikathode in einem Siegbanschen Metallrohr mit ungefähr 50 Kilovolt Spannung und 10 Milliampère Stromstärke geliefert.

Bei der Aufnahme von Pulverphotogrammen, die in der Regel 5 bis 6 Stunden exponiert worden sind, wurden Cameren mit 55 mm Durchmesser angewendet. Für die Drehcamera ist der Durchmesser $63 \mathrm{~mm}$. Sowohl die letztere als auch Präzisionscameren verschiedener Art sind von Phragmén und Westarenen ${ }^{2}$ konstruiert. Die neuesten sind nach dem von $\mathrm{H}$. BoHLIN ${ }^{3}$ angegebenem Prinzip gebaut, und Filme werden anstatt Platten angewendet. Sie sind kalibriert worden mit Photogrammen teils von Kochsalz und teils von einem kubischen Eisen-Wolfram-Carbid, das ein besonders linienreiches Photogramm ergibt, das sich daher besonders gut zum Aufzeichnen der Kalibrierungskurve eignet.

\section{Material.}

FeS. a) Magnetkies: Kongsberg (Kristalle, hexagonale Tafeln), Adolfsgrube, Sudbury, Smörvik, Gjerrestad und Freiberg (derbe Masse).

Analysen von den drei ersteren sind in HinTzes Handbuch aufgenommen, der letztgenannte enthält nach Analyse von G. Lindström $60.18 \% \mathrm{Fe}, 38.88 \% \mathrm{~S}, \mathrm{SiO}_{2} 0.57$, $\mathrm{CaCO}_{3} 0.30$, Summa 99.93 .

b) Troilit:

$\alpha)$ meteorischer: Gibeon, San Angelo, Mount Stirling, Augustinowka und Seeläsgen. Analyse von letzterem ist in Hintzes Handbuch zu finden. Von grossem Interesse ist der Gibeon-Troilit, dessen Photogramm von denjenigen aller anderen, besonders wegen der abnormen Breite der Linien, verschieden ist (Tafel 4

1 G. F. F. Bd. 40, 861 und Bd 44, 197.

2 Jernk. annaler 1923, 449 und 459.

3 Annalen der Physik 61, 1920, 421. 
Fig. 3). Der Bau des Eisens in diesem Meteoriten scheint auch eigentümlicher Art zu sein, wie RINNE ${ }^{1}$ gezeigt hat.

$\beta)$ tellurischer: aus Del Norte County, California. Analyse von EakLe: ${ }^{2}$ Fe $62.7 \%$, S $35.4 \%$, Summa $98.1 \%$.

c) Künstliche Produkte.

$\alpha)$ mit Schwefelüberschuss.

Diese sind nach drei Methoden dargestellt worden:

1) Synthese aus Eisen- und Schwefelpulver mit grossem Überschuss an Schwefel.

2) Glühen von gefälltem Sulfid in Schwefelwasserstoffatmosphäre. Hierbei beobachtet man, dass wenn die Temperatur verhältnismässig niedrig gehalten wird, das Produkt $\mathrm{FeS}_{2}$ gebildet wird, das Photogramme ergibt, die identisch mit denjenigen des Pyrits sind.

Die Analyse von einem mit $\mathrm{FeS}_{2}$ vermengten Produkt ergab Fe $56.5 \%$.

Anm. Die Pulverphotogramme zeigen, dass der Kristallaufbau des gefällten Sulfids vor der Glühung ein ganz anderer als nach derselben ist. Wahrscheinlich erhält man bei der Fällung ein Hydrat von FeS. Dieselbe Verbindung entsteht, wenn man eine innige Mischung von Fe- und S-Pulver mit etwas Wasser vermengt.

3) Glühen von pulverisiertem Pyrit in Schwefelwasserstoffatmosphäre.

$\beta)$ mit Eisenüberschuss.

1) Dieses Produkt ist durch Synthese aus Eisen- und Schwefelpulver mit variierendem Überschuss des ersteren dargestellt worden. Einige der erhaltenen Produkte sind zur Homogenisierung in einem elektrischen Kohlenrohrofen geschmolzen worden. Eine Schmelze, von welcher nach Erstarrung ein Photogramm aufgenommen wurde (Tab. 3), hat einen Fe-Gehalt von $65.2 \%$ LOEBE und BeCKER ${ }^{3}$ haben gezeigt, dass drei Phasen in solchen erstarrten Schmelzen sich vorfinden, nämlich $\mathrm{Fe}$, FeS und ausserdem eine sauerstoffhaltige Phase.

1 Neues Jahrb. für Min. etc. 1910, 1, 115.

2 Op. cit.

3 Z. anorg. Chemie 1912, 76, 264, Taf. 22 und 23. 
Die Pulverphotogramme offenbaren ausser FeS auch $\mathrm{Fe}$ und noch eine unbekannte Phase durch gewisse Linien, die sich auch bisweilen dem Aussehen nach - sie sind nämlich punktiert - von den übrigen Linien unterscheiden. Bei grossem Überschuss an Schwefel braucht man eine derartige Verunreinigung nicht zu befürchten, und Extralinien kommen auf ihren Photogrammen auch nicht vor.

2) Wenn man Pyrit in Wasserstoffgas glüht, entsteht eine Substanz, die mit den vorigen im ganzen identische Photogramme ergibt.

NiS. a) Millerit von Wissen a. d. Sieg und Müsen, letzteres etikettiert als Beyrichit. Nadeln, bis haarfeine Kristalle.

b) Künstliche Produkte.

1) Synthese aus Nickel- und Schwefelpulver mit Überschuss von Schwefel.

2) Glühen in $\mathrm{H}_{2} \mathrm{~S}$-Atmosphäre von gefälltem Sulfid $[39.6 \% \mathrm{~S}]$.

Millerit $[34.5 \% \mathrm{~S}]$,

$\mathrm{Ni}_{3} \mathrm{~S}_{2}[37.4 \% \mathrm{~S}]$.

Anm. Der Bau des gefällten Sulfids ist vor der Glühung sowohl von dem des Millerits als auch von dem des Magnetkieses ganz verschieden, wie es durch Vergleich der Pulverphotogramme hervorgeht.

$\mathbf{N i}_{3} \mathbf{S}_{2}$, durch Schmelzung in Kohlenrohrofen von synthetischem NiS dargestellt. S-Gehalt $=26.4 \%$.

$\operatorname{CoS}=\operatorname{NiS}$ b) $\beta$ ) 1).

(Ni, Fe)S. a) Pentlandit.

a) Mineral aus Sudbury und Beiern. Analysen siehe Hintzes Handbuch.

B) Künstliche Produkte.

1) Synthese aus 4.2 gr. Ni, 4.2 Fe und 5.6 S. Das erhaltene Produkt wurde in einem Kohlenrohrofen geschmolzen, Temp. c:a 1,300 C. Analyse: S $34.0 \%$, $\mathrm{Ni} 32.9 \%, \mathrm{Fe} 33.8 \%$.

2) Synthese von 5.6 gr. Ni, 2.8 $\mathrm{Fe}$ und $5.3 \mathrm{~S}$. Weiter $=\alpha$.

b) Mischkristall, erhalten durch Glühen in $\mathrm{H}_{2} \mathrm{~S}$-A tmosphäre vom

a) Sulfid, das aus einer Mischung von äquivalenten 
Mengen $\mathrm{FeSO}_{4}$ und $\mathrm{NiSO}_{4}$ mit Schwefelammonium gefällt war. Analyse: S $38.4 \%, \mathrm{Ni} 32.8 \%$, Fe (Diff.) $28.7 \%$.

$\beta)$ Pentlandit von a) $\beta$ ) 1). Analyse: S $37.8 \%, \mathrm{Fe}$ 33.9, Ni (Diff.) $28.3 \%$.

(Fe, Co)S, analog mit dem Vorhergehenden b) $\alpha$ ) dargestellt.

FeSe.

1) durch Synthese aus Fe und Se-Pulver mit Überschuss des letzteren erhalten $[35.0 \% \mathrm{Fe}]$.

2) durch Glühen von a) in Wasserstoffatmosphäre $[39.4 \% \mathrm{Fe}]$.

$\mathbf{F e}(\mathbf{S}, \mathbf{S e})$, durch Synthese mit geringem Eisenunterschuss dargestellt, danach in Wasserstoffatmosphäre geglüht. Analyse: Fe $49.8 \%$, S $12.0 \%$, Se (Diff.) $38.2 \%$.

NiSe, analog mit FeSe b) dargestellt.

NiSb. a) Breithauptit aus Andreasberg, hexagonale Tafeln, die in Calcit sitzen. Analyse siehe Hintzes Handbuch.

b) Künstliches Produkt, durch Synthese in Wasserstoffatmosphäre aus Ni- und Sb-Pulver dargestellt.

CuS. a) Covellin aus Alghero, Sardinien; derbe Masse mit dünnen, hexagonal ausgebildeten Tafeln.

b) Gefälltes Sulfid, in $\mathrm{H}_{2} \mathrm{~S}$-Atmosphäre schwach geglüht.

\section{Magnetkies.}

Drehphotogramme. Von einem kleinen Kristall von Kongsberg, der ausser der Basis ein Prisma zeigt, sind drei Arten von Drehphotogrammen aufgenommen worden, nämlich mit der Drehungsachse

a) senkrecht zur Basis (Tafel 1). Dies gibt den Identitätsabstand in der Richtung der c-Achse $=5.72 \AA$. E.

b) parallel mit der Basiskante. Hieraus erhält man den Identitätsabstand in dieser Richtung $=3.48 \AA$. E., und endlich

c) parallel mit der kürzeren Basisdiagonale, woraus der Identitätsabstand in der Richtung derselben $=6.06 \AA$. E. erhalten wird. Dies ist $=3.49 \cdot \sqrt{3}$.

So ist nach diesem Befunde, wenn man die Achsen des Elementarparallelepipeds zu Grunde legt, das obengenannte Prisma (1010). Aus den c- und a-Werten wird das A.chsenverhältnis zu 1,64 berechnet. Der von SeligmanN ${ }^{1}$ durch genaue Kristallmessungen erhaltene Wert ist 1.650. Dieses Resultat wird von Busz ${ }^{2}$ bestätigt,

1 Z. Kryst. 1886, 11.

2 Neues Jahrbuch für Min. etc. 1895, 1, 124. 
Bd 47. H 1.$]$ UNTERSUCHUNG GEWISSER KRISTALLSTRUKTUREN.

der gleichzeitig bemerkt, dass die Messungen von Rose sehr variieren und das von ihm berechnete Achsenverhältnis $1.740 \mathrm{zu}$ hoch ist.

Tab. 1 enthält die aus den Drehphotogrammen abgeleiteten $\sin ^{2} \frac{\boldsymbol{\vartheta}}{2}$.

Tab. 1. Drehphotogramm von Magnetkies.

\begin{tabular}{|c|c|c|c|c|c|c|c|c|c|}
\hline \multirow[b]{3}{*}{ 0-Schicht } & \multicolumn{3}{|c|}{ Drehachse || einer Basis- } & \multicolumn{3}{|c|}{$\begin{array}{c}\text { Drehachse } \| \text { einer Basis- } \\
\text { diagonal }\end{array}$} & \multicolumn{3}{|c|}{$\begin{array}{c}\text { Drehachse senkrecht } \\
\text { zur Basis }\end{array}$} \\
\hline & $\mid \begin{array}{c}\text { Indices, der } \\
\text { dritte ist } \\
\text { fortgelassen }\end{array}$ & $\left|\begin{array}{c}\text { Schwär- } \\
\text { zung }\end{array}\right|$ & $\sin ^{2} \frac{9}{2}$ & Indices & $\begin{array}{l}\text { Schwär- } \\
\text { zung }\end{array}$ & $\sin ^{2} \frac{9}{2}$ & Indices & $\begin{array}{c}\text { Schwär- } \\
\text { zung }\end{array}$ & $\sin ^{2} \frac{\gamma}{2}$ \\
\hline & \begin{tabular}{|c|}
001 \\
100 \\
101 \\
102 \\
$\beta 201,103$ \\
201 \\
202 \\
104 \\
203 \\
105 \\
300
\end{tabular} & $\begin{array}{c}\operatorname{seh} w \\
s t \\
m \\
m \\
\operatorname{seh} w+ \\
s t . \\
\operatorname{seh} w+ \\
\operatorname{seh} w \\
\operatorname{sen} w \\
\operatorname{sch} w \\
s t\end{array}$ & $\begin{array}{l}0.030 \\
0.108 \\
0.133 \\
0.227 \\
0.382 \\
0.467 \\
0.547 \\
0.572 \\
0683 \\
0.830 \\
0,953\end{array}$ & $\begin{array}{c}001 \\
100 \\
\beta 110 \\
110 \\
\beta 201,103 \\
201 \\
211\end{array}$ & $\begin{array}{l}\operatorname{schw} \\
\text { m } \\
\operatorname{sch} w \\
\text { st } \\
\text { schw } \\
\text { st } \\
\text { st }\end{array}$ & $\begin{array}{l}0.026 \\
0.107 \\
0.252 \\
0.309 \\
0.369 \\
0.448 \\
0.763\end{array}$ & $\begin{array}{c}\beta 100 \\
100 \\
\beta 110 \\
110 \\
200 \\
210 \\
\beta 300 \\
300\end{array}$ & $\begin{array}{l}\operatorname{sch} w \\
\text { m } \\
\text { schw } \\
\text { st } \\
\text { schw } \\
\text { schw } \\
\text { schw } \\
\text { st }\end{array}$ & $\begin{array}{l}0.091 \\
0.110 \\
0.268 \\
0.325 \\
0.434 \\
0.742 \\
0.781 \\
0.941\end{array}$ \\
\hline 1-Schicht & $\begin{array}{l}100 \\
101 \\
102 \\
110 \\
103 \\
104\end{array}$ & $\begin{array}{c}\mathrm{m} \\
\operatorname{sch} w \\
\mathrm{st} \\
\mathrm{st} \\
\operatorname{sch} w \\
\operatorname{sch} w\end{array}$ & $\begin{array}{l}0.117 \\
0.147 \\
0.225 \\
0.320 \\
0.382 \\
0.575\end{array}$ & $\begin{array}{c}100 \\
101 \\
102 \\
103 \\
104 \\
211,114 \\
105 \\
212\end{array}$ & $\begin{array}{c}\mathrm{m} \\
\mathrm{m} \\
\mathrm{m} \\
\mathrm{m} \\
\mathrm{m}- \\
\mathrm{m}+ \\
\mathrm{m} \\
\mathrm{m}+\end{array}$ & $\begin{array}{l}0.107 \\
0.130 \\
0.217 \\
0.364 \\
0.563 \\
0.766 \\
0.814 \\
0.845\end{array}$ & $\begin{array}{l}101 \\
201 \\
211\end{array}$ & $\begin{array}{l}\text { st } \\
\text { m } \\
\text { st }\end{array}$ & $\begin{array}{l}0.140 \\
0.457 \\
0.773\end{array}$ \\
\hline 2-Schicht & & & & & & & $\begin{array}{l}102 \\
202\end{array}$ & $\begin{array}{l}\text { st } \\
\text { m } \\
\text { st }\end{array}$ & $\begin{array}{l}0.229 \\
0.549 \\
0.862\end{array}$ \\
\hline
\end{tabular}

Wenn auch die Werte nicht so genau sind, so sind sie doch von grosser Bedeutung bei Indizierung der Pulverphotogramme. Eine Anomalie liegt bei den Drehphotogrammen vor, indem der Reflex von 0001 da vorkommt, aber nicht in den Pulverphotogrammen.

Lauephotogramm. Nach vielen vergeblichen Versuchen mit der Strahlenrichtung senkrecht zur Basis erhielt Verf. ein Photogramm von einem sehr kleinen Kongsbergskristall, das sich als einheitlich erwies. Eine Kopie desselben ist in Tafel 2 beigefügt. Es besitzt volle hexagonale Symmetrie. Von dem Lauephotogramm ist eine gnomonische Projektion (Fig. 1) ausgeführt. Aus der Projektion wird das Achsenverhältnis = 1.69 gemessen.

Da der Abstand vom Zentralfleck bis zum nächsten Reflexpunkt 
$0.85 \mathrm{~cm}$ und der Abstand Kristall-Platte $3.26 \mathrm{~cm}$ beträgt, wird der kleinste Glanzwinkel $\frac{\vartheta}{2}=7,3$. Hieraus c $\sim 5.6 \AA$ A. E., wenn $\lambda_{\min }=0.18$ A. E. gesetzt wird.

Der hier erhaltene approximative Wert für c stimmt so genau, wie man zu erwarten berechtigt ist, mit dem aus dem Drehphotogramm erhaltenen überein.

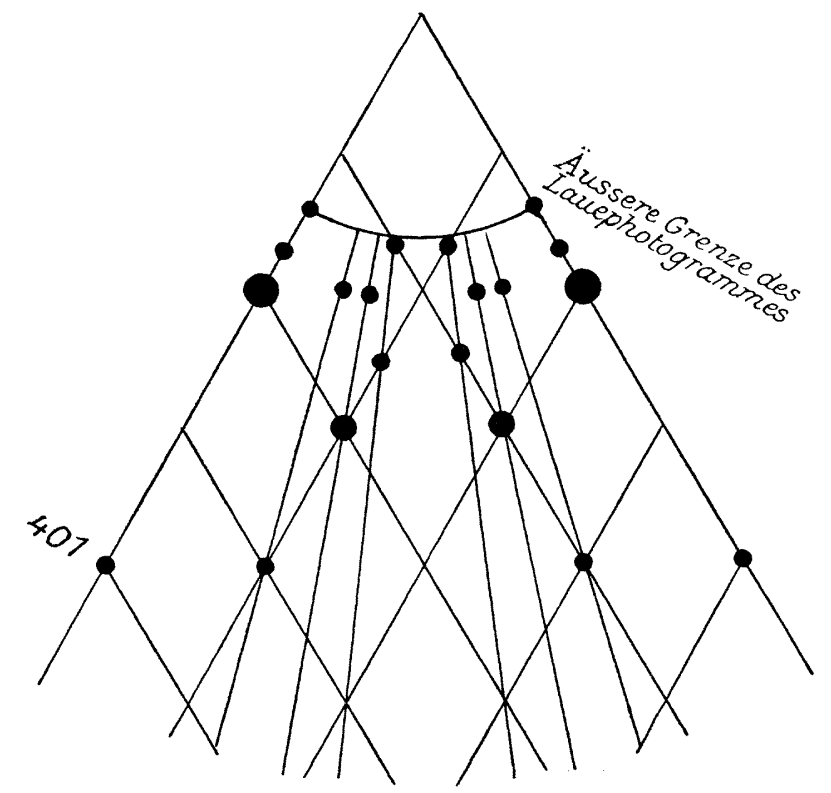

Fig. 1. Gnom. Proj, von Magnetkies nach Basis $(\mathrm{r}=1 \mathrm{~cm})$.

Pulverphotogramme. Pulverphotogramme sind von sowohl verschiedenen Magnetkiesen und künstlichen Produkten als auch von Troiliten, und zwar teils siderischen und teils einem tellurischen (California), aufgenommen worden ('Tab. 2 und 3). Alle haben im grossen ganzen gleiche Photogramme ergeben.

Aus den Tabellen über die Pulverphotogramme und aus Tafel 4 geht indessen hervor, dass eine Verschiebung gewisser Linien der Photogramme mit erhöhtem Fe-Gehalt stattfindet. Am stärksten treten die Abweichungen bei gewissen Linien hervor, nämlich bei denjenigen, welche Flächen mit hohem letzten Index entsprechen. So zum Beispiel gibt die Fläche $105^{2}$ bei den Magnetkiesen

1 NiggLi: Gtom. Krist. des Diskontinuums 512.

${ }^{2} \mathrm{Im}$ folgenden wird der dritte hexagonale Index weggelassen. 
Tab. 2. Pulverphotogramme von FeS mit Scwefeliiberschuss.

\begin{tabular}{|c|c|c|c|c|c|c|c|c|c|c|c|}
\hline \multirow{5}{*}{\multicolumn{2}{|c|}{ Indices }} & \multirow{3}{*}{\multicolumn{3}{|c|}{$\begin{array}{l}\text { Magnetkies: } \\
\text { Kristalle aus } \\
\text { Kongsberg }\end{array}$}} & \multicolumn{7}{|c|}{$\mathrm{Künstliches} \mathrm{FeS,} \mathrm{durch}$} \\
\hline & & & & & \multirow{2}{*}{\multicolumn{2}{|c|}{$\begin{array}{l}\text { Synthese mit } \\
\text { Schwefelüber. } \\
\text { schuss }\end{array}$}} & \multicolumn{5}{|c|}{ Glïhen in $\mathrm{H}_{2} \mathrm{~S} \cdot$ Atmosphäre von } \\
\hline & & & & & & & $\begin{array}{l}\text { gefäl } \\
\text { Sul }\end{array}$ & & Pyrit & aus $\mathrm{K}$ & leva \\
\hline & & \multirow{2}{*}{\multicolumn{2}{|c|}{$\begin{array}{c}\text { Schwär- } \\
\text { zung }\end{array}$}} & \multirow{2}{*}{$\begin{array}{l}\sin ^{2} \frac{7}{2} \\
\text { gem. }\end{array}$} & \multirow{2}{*}{$\begin{array}{c}\text { Schwär- } \\
\text { zung }\end{array}$} & \multirow{2}{*}{$\begin{array}{l}\sin ^{2} \frac{7}{2} \\
\text { gem. }\end{array}$} & \multirow{2}{*}{$\begin{array}{c}\text { Schwär- } \\
\text { zung }\end{array}$} & \multirow{2}{*}{$\begin{array}{l}\sin ^{2} \frac{y}{2} \\
\text { gem. }\end{array}$} & \multirow{2}{*}{$\begin{array}{c}\text { Schwär- } \\
\text { zung }\end{array}$} & \multicolumn{2}{|c|}{$\sin ^{2} \frac{\vartheta}{2}$} \\
\hline & & & & & & & & & & $\begin{array}{l}\text { gemes- } \\
\text { sen }\end{array}$ & $\begin{array}{l}\text { berech- } \\
\text { net }^{1}\end{array}$ \\
\hline$\beta 100$ & & & & & & & & & $\operatorname{sch} w-$ & 5 & 87 \\
\hline 100 & 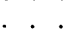 & & $\operatorname{sch} w$ & 0.102 & $\mathrm{~m}$ & 0.106 & $\mathrm{~m}-$ & 0.105 & $\mathrm{~m}$ & & \\
\hline$\beta 101$ & .5 & & & & & & & & $\operatorname{sch} w$ & & 11 \\
\hline 101 & . . & & $\mathrm{m}$ & 0.133 & $\mathrm{~m}$ & 0.136 & $\mathrm{~m}$ & 0.135 & . & & 0 \\
\hline$\beta 102$ & 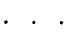 & & $\operatorname{seh} w$ & 0.181 & $\operatorname{seh} w$ & 0.185 & $\operatorname{sch} w+$ & 0.182 & $\mathrm{~m}$ & 9 & 83 \\
\hline 102 & ${ }^{\circ}$ & & st & 0.221 & st & 0.2 & st + & 0.223 & st + & & 21 \\
\hline$\beta 110$ & . & & $\operatorname{schw}$ & 0.268 & $\operatorname{seh} w$ & 0.26 & $\operatorname{sch} w$ & 0.264 & $\operatorname{sch} w+$ & 31 & 0.262 \\
\hline$\beta 103$ & 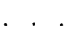 & & $\operatorname{seh} w$ & 0.301 & & & & & $\operatorname{sch} w-$ & 01 & 0.302 \\
\hline 110 & . & & $\mathrm{m}$ & 0.317 & st & 0.322 & st & 0.321 & st & & 18 \\
\hline 103 & & & $\operatorname{sch} w$ & 0.365 & $\operatorname{sch} w$ & 0.369 & schw & 0.369 & $m-$ & 0.2 & 0.366 \\
\hline 200 & $\cdot$ & & & & $\operatorname{sch} w-$ & 0.432 & & & $\operatorname{seh} w$ & 0.423 & 0.424 \\
\hline$\beta 202$ & . & & & & & & & & & & 0.445 \\
\hline 201 & & & $\operatorname{seh} w$ & 0.451 & $\mathrm{~m}$ & 0.461 & $\operatorname{seh} w$ & 0.464 & $\operatorname{seh} w+$ & 0.452 & 0.453 \\
\hline 004 & · & & $m$ & 0.539 & st & 0.5 & & & $\underset{\text { st }}{\operatorname{seh} w}+$ & & \\
\hline 104 & $\cdot$ & & $\operatorname{sch} w$ & 0.563 & $\operatorname{seh} w-$ & 0.572 & schw & 0.568 & schw & & \\
\hline$\beta 211$ & & & & & & & & $0 \circ$ & & & 0.636 \\
\hline$\beta 114$ & 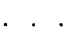 & & & & & & $\mathrm{s}$ & 14 & $\operatorname{sen} w$ & 12 & 0.643 \\
\hline 203 & - & & $\operatorname{schw}$ & 0.683 & $\operatorname{sch} w$ & 0.689 & $\operatorname{seh} w$ & 0.684 & $\operatorname{seh} w+$ & 0.682 & 0.684 \\
\hline$\beta 212$ & . & & & & $\operatorname{sch} w-$ & 0.710 & $\operatorname{sen} w-$ & 0.707 & $\operatorname{sch} w$ & 0.702 & 0.707 \\
\hline 210 & & & & & & & & & $\operatorname{seh} w$ & $0.74 t$ & \\
\hline 211 & . & • & \} & 0.772 & st & 0.782 & st + & 0.778 & st & 0.778 & 0.771 \\
\hline$\beta 300$ & & & & & & & & & & & 0.787 \\
\hline 105 & • & & & & $\operatorname{sch} w$ & 0.8 & $\operatorname{sch} w$ & 0.825 & schw & 0829 & 0.828 \\
\hline 212 & . & & $\mathrm{m}$ & 0.854 & st & 0.860 & st & 0.855 & st & 0.857 & 0.858 \\
\hline 204 & . . & & & & & & & & $\operatorname{sch} w-$ & 0.87 & 0.886 \\
\hline 300 & 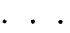 & & & & $\mathrm{m}$ & 0.951 & $\operatorname{seh} w+$ & 0.951 & st & 0.949 & 0.954 \\
\hline
\end{tabular}

${ }^{1}$ Aus folgender Formel:

$$
\begin{aligned}
& \mathrm{Fe}_{K \alpha}: \sin ^{2} \frac{\vartheta}{2}=0.1060\left(\mathrm{~h}^{2}+\mathrm{hk}+\mathrm{k}^{2}\right)+0.0289 \mathrm{l}^{2} \\
& \mathrm{Fe}_{K \beta}: \sin ^{2} \frac{\vartheta}{2}=0.0874\left(\mathrm{~h}^{2}+\mathrm{hk}+\mathrm{k}^{2}\right)+0.0238 \mathrm{l}^{2}
\end{aligned}
$$

und den synthetischen Produkten mit Schwefelüberschuss $\sin ^{2} \frac{\boldsymbol{9}}{2}=$ ungefähr 0.825, dagegen erhält man für die Troiliten und die synthetischen Produkte mit Fe-Überschuss einen bedeutend niedrigeren Wert (etwa 0.795). Sehr deutlich tritt dies auch auf Tafel 4 hervor, wo der Reflex von 102 merklich verschoben ist. Flächen 
Tab. 3. Pulverphotogramm von FeS mit Eisenüberschuss.

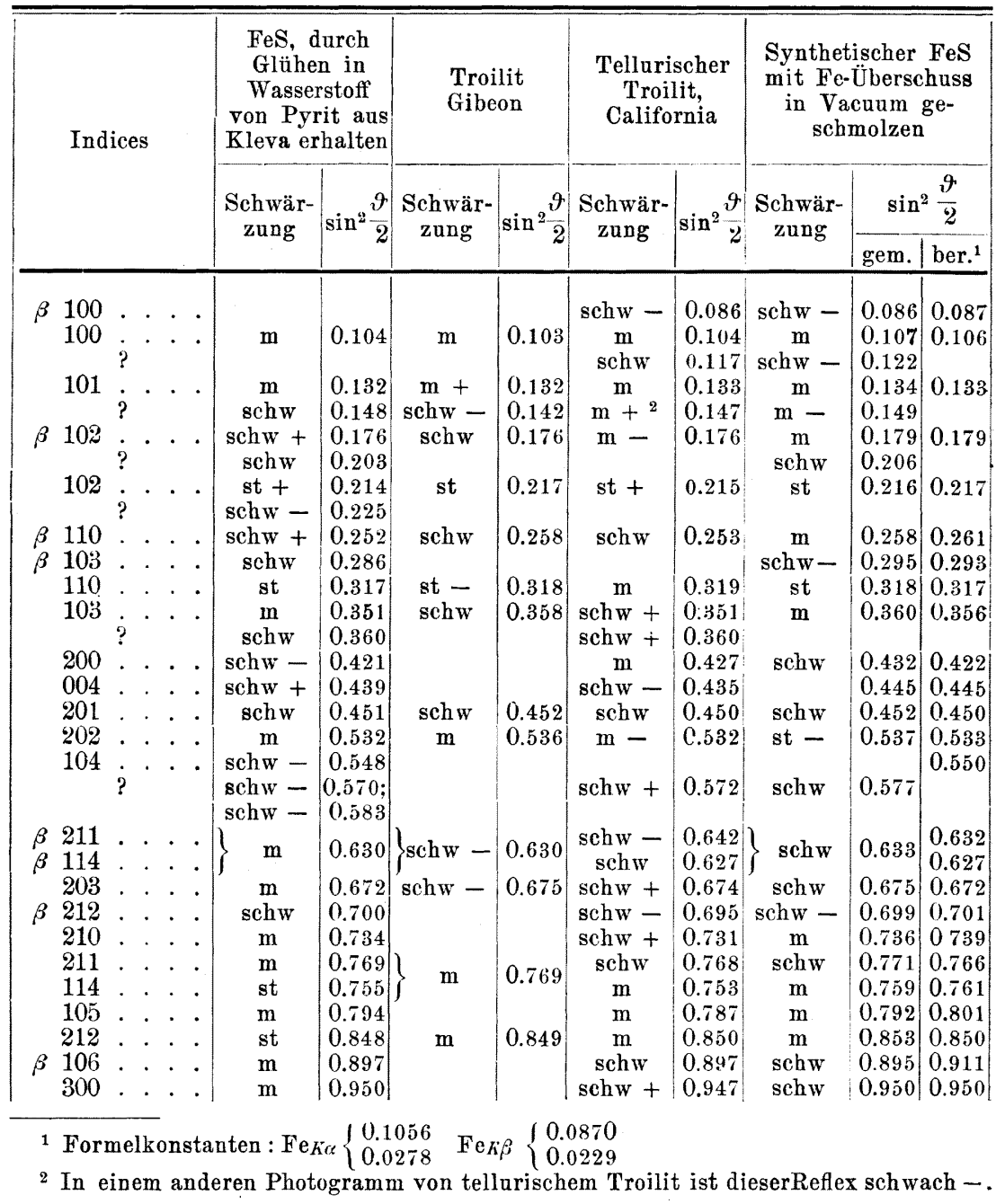

mit letztem Index $=0$ zeigen dagegen keine Verschiebung. So gibt $110 \sin ^{2} \frac{9}{2}$ ungefähr 0.320 bei allen Photogrammen und 300 $\sin ^{2} \frac{9}{2}$ ungefähr 0.950 .

Die quadratische Formel für Magnetkies und andere Fe S-Produkte mit Schwefelüberschuss wird: $\sin ^{2} \frac{9}{2}=0.1060\left(\mathrm{H}^{2}+\mathrm{HJ}+\mathrm{J}^{2}\right)+$ 
$+0.0289 \mathrm{~L}^{2}$, wo $0.1060=\frac{\lambda^{2}}{3 \mathrm{a}^{2}}$ und $0.0289=\frac{\lambda^{2}}{4 \mathrm{c}^{2}}$ ist. Hieraus erhält $\operatorname{man} \mathrm{a}=3.43^{*}$ und $\mathrm{c}=5.68$ und also $\frac{\mathrm{c}}{\mathrm{a}}=1.66$. Die übrigen $\mathrm{Fe} \mathrm{S}$ Photogramme ergeben niedrigere Formelkonstanten, nämlich 0.1056 , bezw. 0.0278. Aus den letzteren werden $\mathrm{a}=3.43$ und $\mathrm{c}=5.79$ und demnach $\frac{\mathrm{c}}{\mathrm{a}}=1.69$ abgeleitet.

Aus dem Vorstehenden geht hervor, dass mit zunehmendem FeGehalt die grössere Formelkonstante 0.106 sich nahezu unverändert erhält, woraus folgt, dass der Identitätsabstand a konstant ist; die zweite Formelkonstante dagegen nimmt ab, was bedingt, dass $\mathrm{c}$ zunimmt. Der grössere Raum wird in erster Linie durch eine Vergrösserung des Gitters in der Richtung der c-Achse gewonnen, was eine Steigerung des Achsenverhältnisses c:a von 1.66 auf 1.69 bedingt.

Substitution oder Addition der Schwefelatome. Der Schwefelüberschuss des Magnetitkieses kann entweder durch eine Substitution von Fe-gegen S-Atome, oder durch die Eindringung von Schwefelatomen in die Zwischenräume des Gitters entstehen. Wenn auch die spez. Gewichtsbestimmungen von Magnetkies und Troilit grosse Fehler aufweisen, scheint es doch offenbar, dass das spez. Gewicht des Troilits grösser als das des Magnetkieses ist. Da nebenbei die c-Achse bei grösserem Schwefelgehalt abnimmt, während die aAchse sich konstant hält, müssen die S-Atome die Fe-Atome substituieren. Es ist sehr wahrscheinlich, dass die umgekehrte Substitution in eisenreichen Verbindungen vorhanden ist.

Anzahl Moleküle im Elementarparallelepiped. Das Molekulargewicht M für FeS ist $=87.9$. Das Gewicht eines Atomes $\mathrm{H}=$ $1.65 \cdot 10^{-24} \mathrm{gr}$. Das spez. Gewicht $\mathrm{s}$ für Troilit wird $=4.74$ angenommen, was in HiNTzes Handbuch als das Resultat der sichersten Bestimmung angegeben wird. $\mathrm{N}=$ Anzahl Mol. im Elementarparallelepiped. Das Volumen des Elementarparallelepipeds $=$ $\frac{\mathrm{c} \cdot \mathrm{a}^{2} \sqrt{3}}{2}=1.65 \frac{\mathrm{MN}}{\mathrm{s}}$. Hieraus erhält $\operatorname{man} \mathrm{N}=\frac{4.74 \cdot 5.79 \cdot 3.43^{2} \sqrt{3}}{2 \cdot 1.65 \cdot 87.9}=$ $1.93=2$ (approx.). Wird $\mathrm{N}$ exakt $=2$ angenommen, so erhält man das spez. Gewicht $=4.90$, was mit dem angenommenen Wert gut stimmt.

* Die Gittergrössen sind in $\AA$. E. gemessen. 
Strukturbestimmung. Eine sichere morphologische Bestimmung der Symmetrieklasse des Magnetkieses liegt nicht vor. Grotн gibt in seiner Chem. Krystallographie an, dass der Magnetkies dihex. pyr. (?) ist.

Da das Lauephotogramm rom Magnetkies volle hexagonale Symmetrie zeigt, kann derselbe den Symmetrieklassen $\mathrm{D}_{3 \mathrm{~h}}, \mathrm{D}_{6}, \mathrm{C}_{6 \mathrm{r}}$ und $\mathrm{D}_{6 \mathrm{~h}}$ angehören.

Obwohl es nicht a priori evident ist, dass alle Atome von der selben Art gleichwertig sind, ist es doch sehr wahrscheinlich. Darum ist Verf. in der folgenden Diskussion von dieser Voraussetzung ausgegangen.

Folgende 2-zählige Lagen finden sich nach $\mathrm{WrckofF}^{1}$ oder $\mathrm{NIGGLI}^{2}$ in hierher-gehörigen Raumgruppen. $D_{3 \mathrm{~h}}^{1}, D_{3 \mathrm{~h}}^{2}, \mathrm{D}_{3 \mathrm{~h}}^{3}, \mathrm{D}_{3 \mathrm{~h}}^{4}, \mathrm{D}_{6}^{1}, \mathrm{D}_{6}^{6}, \mathrm{C}_{6 \mathrm{v}}^{1}$, $\mathrm{C}_{6 \mathrm{v}}^{2}, \mathrm{C}_{6 \mathrm{v}}^{3}, \mathrm{C}_{6 \mathrm{v}}^{4}, \mathrm{D}_{6 \mathrm{~h}}^{1}, \mathrm{D}_{6 \mathrm{~h}}^{2}, \mathrm{D}_{6 \mathrm{~h}}^{3}, \mathrm{D}_{6 \mathrm{~h}}^{4}$.

Von diesen Strukturen werden in erster Linie diejenigen verworfen, die durch Aufteilung in zwei gleiche Teilstrukturen Elementarparallelepipede mit nur halb so grossem Identitätsabstand in der Richtung der c-Achse wie dem experimentell erhaltenen ergeben. Diese sind $\mathrm{D}_{3 \mathrm{~h}}^{2}, \mathrm{C}_{6 \mathrm{v}}^{2}, \mathrm{C}_{6 \mathrm{v}}^{3}$ und $\mathrm{D}_{6 \mathrm{~h}}^{2}=\mathrm{D}_{6 \mathrm{~h}}^{3}$ und teilweise $\mathrm{D}_{3 \mathrm{~h}}^{4}$ und $\mathrm{C}_{6 \mathrm{v}}^{4}$.

Alsdann erübrigen folgende Möglichkeiten, wobei Verf. beim Berechnen der Strukturfaktoren die zwei ersten Koordinaten für die Fe-Atome angenommen hat.

$\mathbf{D}_{3 \mathbf{3}}^{\mathbf{1}} \quad$ 1) $00 \mathrm{p}, 00 \overline{\mathrm{p}} ; 00 \mathrm{q}, 00 \overline{\mathrm{q}}$; der Strukturfaktor $\mathrm{A}^{2}=4(\mathrm{Fe} \cos 2 \pi \mathrm{pL}+$ $+\mathrm{S} \cos 2 \pi q \mathrm{~L})^{2}$.

2) $00 \mathrm{p}, 00 \overline{\mathrm{p}} ; \frac{2}{2} \frac{1}{3} \mathrm{q} \cdot \frac{2}{3} \frac{1}{3} \overline{\mathrm{q}} \cdot \mathrm{A}^{2}=4\left(\mathrm{Fe}^{2} \cos ^{2} 2 \pi \mathrm{pL}+\mathrm{S}^{2} \cos ^{2} 2 \pi \mathrm{qL}+\right.$ $\left.+2 \mathrm{FeS} \cos 2 \pi \mathrm{pL} \cos 2 \pi \mathrm{qL} \cdot \cos 2 \pi \frac{2 \mathrm{H}+\mathrm{I}}{3}\right)$.

3) $\frac{2}{3} \frac{1}{3} \mathrm{p}, \frac{2}{3} \frac{1}{3} \overline{\mathrm{p}} ; \frac{1}{3} \frac{2}{3} \mathrm{q}, \frac{1}{3} \frac{2}{3} \bar{q}$.

Wird origo nach dem Punkt $\frac{2}{3} \frac{1}{3} 0$ verlegt, so erhält man $00 \mathrm{p}$, $00 \overline{\mathrm{p}} ; \frac{2}{3} \frac{1}{3} \mathrm{q}, \frac{2}{3} \frac{1}{3} \overline{\mathrm{q}}$. Also gleich dem Vorhergehenden.

$\left.\mathbf{D}_{3 \mathrm{~h}}^{\mathbf{3}}=\mathbf{D}_{6}^{\mathbf{1}}=\mathbf{D}_{6 \mathrm{~h}}^{\mathbf{1}} 1\right)=$ Spezialfall von $\mathrm{C}_{6 \mathrm{v}}^{1}$.

2) $\frac{1}{3} \frac{2}{3} 0, \frac{2}{3} \frac{1}{3} 0 ; 00 \mathrm{p}, 00 \overline{\mathrm{p}} . \mathrm{A}^{2}=4\left(\mathrm{Fe}^{2} \cos ^{2} 2 \pi \frac{\mathrm{H}-\mathrm{I}}{6}+\mathrm{S}^{2} \cos ^{2} 2 \pi \mathrm{pL}+\right.$

1 The analytical expression of the results of the theory of space-groups.

2 Op. cit. 
$\left.+2 \mathrm{FeS} \cos 2 \pi \mathrm{pL} \cos 2 \pi \frac{\mathrm{H}+\mathrm{I}}{2} \cos 2 \pi \frac{\mathrm{H}-\mathrm{I}}{6}\right)$.

$\mathbf{D}_{3 \mathbf{h}}^{4}=\mathbf{D}_{6 \mathrm{~h}}^{\mathbf{4}} \quad$ 1) $000,00 \frac{1}{2} ; \frac{1}{3} \frac{2}{3} \frac{1}{4}, \frac{2}{3} \frac{1}{3} \frac{3}{4} . \quad$ L ungerade: $\mathrm{A}^{2}=$

$=4 \mathrm{~S}^{2} \cos ^{2} 2 \pi\left(\frac{\mathrm{H}-\mathrm{I}}{6}+\frac{\mathrm{L}}{4}\right)$. L gerade: $\mathrm{A}^{2}=4\left[\mathrm{Fe}^{2}+\mathrm{S}^{2} \cos ^{2} 2 \pi \frac{\mathrm{H}-\mathrm{I}}{6}+\right.$ $\left.+2 \mathrm{FeS} \cos 2 \pi\left(\frac{\mathrm{H}-\mathrm{I}}{6}+\frac{\mathrm{L}}{4}\right) \cos 2 \pi \frac{\mathrm{H}+\mathrm{I}}{2}\right]$.

2) $00 \frac{1}{4}, 00 \frac{3}{4} ; \frac{1}{3} \frac{2}{3} \frac{1}{4}, \frac{2}{3} \frac{1}{3} \frac{3}{4}$. L ungerade: $\mathrm{A}^{2}=4 \mathrm{~S}^{2} \sin ^{2} 2 \pi \frac{\mathrm{H}-\mathrm{I}}{6}$

$\mathrm{L}$ gerade: $\mathrm{A}^{2}=4\left(\mathrm{Fe}^{2}+\mathrm{S}^{2} \cos ^{2} 2 \pi \frac{\mathrm{H}-\mathrm{I}}{6}+2 \mathrm{FeS} \cos 2 \pi \cdot \frac{\mathrm{H}+\mathrm{I}}{2}\right.$. $\left.\cdot \cos 2 \pi \frac{\mathrm{H}-\mathrm{I}}{6}\right)$.

3) $\frac{1}{3} \frac{2}{3} \frac{1}{4}, \frac{2}{3} \frac{1}{3} \frac{3}{4} ; \frac{1}{3} \frac{2}{3} \frac{3}{4}, \frac{2}{3} \frac{1}{3} \frac{1}{4}$. L ungerade: $\mathrm{A}^{2}=$ $=4(\mathrm{Fe}-\mathrm{S})^{2} \sin ^{2} 2 \pi \frac{\mathrm{H}-\mathrm{I}}{6}$. L gerade: $\mathrm{A}^{2}=4(\mathrm{Fe}+\mathrm{S})^{2} \cos ^{2} 2 \pi \frac{\mathrm{H}-\mathrm{I}}{6}$. $\mathrm{C}_{6 \mathrm{r}}^{1}$ 1) $\frac{1}{3} \frac{2}{3} 0, \frac{2}{3} \frac{1}{3} 0 ; \frac{1}{3} \frac{2}{3} \mathrm{p}, \frac{2}{3} \frac{1}{3} \mathrm{p} . \quad \mathrm{A}^{2}=4 \cos ^{2} 2 \pi \frac{\mathrm{H}-\mathrm{I}}{6}\left[\mathrm{Fe}^{2}+\mathrm{S}^{2}+\right.$ $+2 \mathrm{FeS} \cos 2 \pi \mathrm{pL}]$.

$\mathbf{C}_{6 \mathbf{r}}^{4}$ 1) $\frac{1}{3} \frac{2}{3} 0, \frac{2}{3} \frac{1}{3} \frac{1}{2} ; \frac{1}{3} \frac{2}{3} \mathrm{p}, \frac{2}{3} \frac{1}{3}\left(\mathrm{p}+\frac{1}{2}\right) . \quad \mathrm{A}^{2}=4 \cos ^{2} 2 \pi\left(\frac{\mathrm{H}-\mathrm{I}}{6}+\frac{\mathrm{L}}{4}\right)$. $\left[\mathrm{Fe}^{2}+\mathrm{S}^{2}+2 \mathrm{Fe} \mathrm{S} \cos 2 \pi \mathrm{pL}\right]$.

2) $000,00 \frac{1}{2} ; \frac{1}{3} \frac{2}{3} p, \frac{2}{3} \frac{1}{3}\left(p+\frac{1}{2}\right)$. L ungerade: $4 \mathrm{~S}^{2} \sin ^{2} 2 \pi \frac{\mathrm{H}-\mathrm{I}}{6}$.

$\mathrm{L}$ gerade: $4\left[\mathrm{Fe}^{2}+\mathrm{S}^{2} \cos ^{2} 2 \pi \frac{\mathrm{H}-\mathrm{I}}{6}+2 \mathrm{FeS} \cos 2 \pi \frac{\mathrm{H}-\mathrm{I}}{6} \cdot \cos 2 \pi\right.$ $\left.\left(\frac{\mathrm{H}+\mathrm{I}}{2}+\mathrm{pL}\right)\right]$.

Erörterung der Strukturen. Die vorstehenden Strukturen sind mit experimentellen Daten vereinbar, so lange die Intensität der Linien der Photogramme unberücksichtigt gelassen wird. Es ist indessen nur die letztgenannte Struktur, welche auch die Intensitätsverteilung auf den Filmen ausreichend erklärt. Alle übrigen Strukturen geben berechnete Intensitäten, die von den beobachteten abweichen. Dies wird im Detail in der folgenden Darlegung gezeigt.

$\mathbf{D}_{\mathbf{3 h}}^{\mathbf{1}}$ 1) $\mathrm{A}^{2}$ erhält den Max.-Wert für $L=0$, was nicht damit stimmt, dass die Reflexe von $100 \mathrm{~m}$ (mittel), 110 st. (stark), 200

3-250333. G. F. F. 1925. 
schw. (schwach), 210 und 300 st. sind. Ausserdem erhält $A^{2}$ dieselben Werte für dieselben L-Werte, unabhängig von den übrigen Indices, was den Berbachtungen widerspricht. Demnach ist 001 schw (Drehfilm) oder nicht vorhanden (Debyefilm) 101 st, 111 fehlt usw. Der Raum, der jedem Atom in dieser Struktur zur Verfügung steht, ist zu klein, da alle Atome auf derselben Achse sitzen. Auch deswegen muss dieser Strukturvorschlag verworfen werden.

2) $\mathrm{A}^{2}$ erhält für gewisse Flächen Werte, die von den Parametern unabhängig sind, wie aus dem ersten Teil der Tabelle 4 ersichtlich. Der berechnete Ausdruck für die Intensität $\frac{f A^{2}}{L o}$, wo $f=$ Frequenzund $L_{0}=$ Lorentzfaktor ist, stimmt einigermassen mit der beobachteten Schwärzung. Aus den $\mathrm{A}^{2}$-Werten für 001, 111 und 301 geht hervor, dass $\cos 2 \pi p$ und $\cos 2 \pi q$ verschiedene Zeichen haben, aber numerisch gleich sein müssen, damit $\mathrm{A}^{2}$ gleich 0 werden soll. In gleicher Weise geht aus den $\mathrm{A}^{2}$-Werten für 002, 112 und 302, welche Reflexe auf dem Film fehlen, hervor, dass $\cos 2 \pi \cdot 2 p$ und $\cos 2 \pi \cdot 2 q$ numerisch gleich gross sein müssen, aber mit verschiedenen Zeichen. Schliesslich sollen die Strukturfaktoren für 003, 113 klein $(0)$ sein, was erfordert, dass $\cos 2 \pi \cdot 3 p$ und $\cos 2 \pi \cdot 3 q$

Tab. 4.

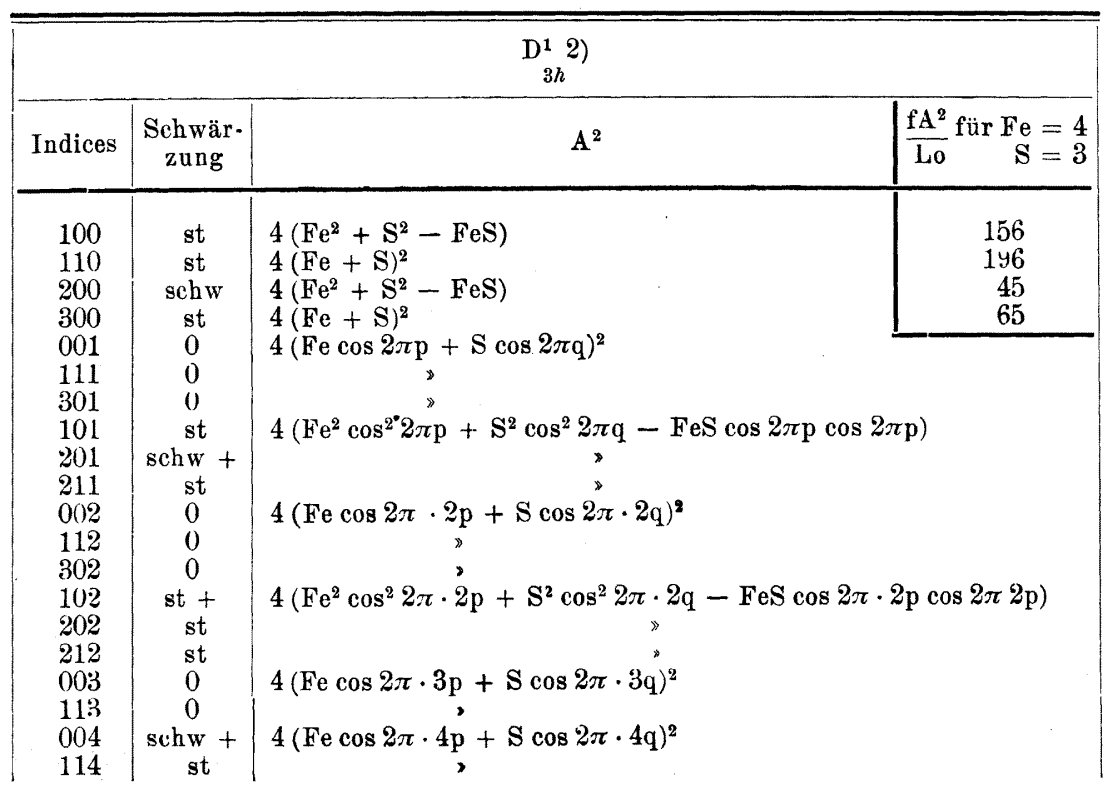


Bd 47. H. 1.] UNTERSUCHUNG GEWISSER KRISTALLSTRUKTUREN.

auch numerisch nahezu gleich gross sein sollen mit verschiedenen Zeichen. Aber die Strukturfaktoren für 004 und 114 dürfen nicht 0 werden.

Diese Anforderungen werden nur erfüllt, wenn $p=\frac{1}{8}$ und $q=\frac{3}{8}$ ist. Dann wird nämlich $\cos 2 \pi \cdot p=\sqrt{\frac{1}{2}}, \cos 2 \pi \cdot 2 p=0$, $\cos 2 \pi \cdot 3 p=-\sqrt{\frac{1}{2}}$ und $\cos 2 \pi \cdot 4 p=-1, \cos 2 \pi \cdot q=-\sqrt{\frac{1}{2}}$, $\cos 2 \pi \cdot 2 q=0, \cos 2 \pi \cdot 3 q=\sqrt{\frac{1}{2}}$ und $\cos 2 \pi \cdot 4 q=-1$.

Aber diese Parameter bedingen für den Reflex 102, der st + (sehr stark) ist, den Strukturfaktor 0, für 202 und 212 welche st sind, gleichfalls 0 .

$\mathbf{D}_{3 \mathbf{h}}^{\mathbf{3}} \quad$ 2) Aus der Tabelle 5, nach dem letzten Index gruppiert, geht hervor, dass die Übereinstimmung innerhalb jeder Gruppe recht gut ist. Die Werte für $A^{2}$ in der ersten Gruppe sind unabhängig vom Parameter. Um für die Flächen in der zweiten Gruppe, denen keine Linien entsprechen, den Strukturfaktor $0 \mathrm{zu}$ erhalten, ist erforderlich, dass $\cos 2 \pi \cdot \mathrm{p}$ negativ wird. Aus ähnlichen Gründen ergibt sich, dass $\cos 2 \pi \cdot 2 p$ und $\cos 2 \pi \cdot 3 p$ negativ sein müssen. Einen solchen $\mathrm{p}$-Wert gibt es nicht.

\section{Tab. 5.}

\begin{tabular}{|c|c|c|c|c|c|}
\hline \multicolumn{6}{|c|}{$\underset{3 h}{\left.\mathrm{D}^{3} 2\right)}$} \\
\hline $\begin{array}{c}\text { In- } \\
\text { dices }\end{array}$ & $\begin{array}{c}\text { Schwär- } \\
\text { zung }\end{array}$ & $A^{2}$ & $\begin{array}{c}\text { In- } \\
\text { dices }\end{array}$ & $\begin{array}{c}\text { Schwär- } \\
\text { zung }\end{array}$ & $A^{2}$ \\
\hline 100 & $\mathrm{~m}$ & $(\mathrm{~S}-2 \mathrm{Fe})^{2}$ & 202 & st & $(\mathrm{S}-2 \mathrm{Fe} \cos 2 \pi \cdot 2 n)^{2}$ \\
\hline 110 & st & $4(\mathrm{~S}+\mathrm{Fe})^{2}$ & 212 & st & $(\mathrm{S}-2 \mathrm{Fe} \cos 2 \pi \cdot 2 \mathrm{p})^{2}$ \\
\hline 200 & $\operatorname{sch} w$ & $(\mathrm{~S}-2 \mathrm{Fe})^{2}$ & & & \\
\hline 210 & $\operatorname{schw}$ & $(\mathrm{S}-2 \mathrm{Fe})^{2}$ & 003 & 0 & $4(\mathrm{~S}+\mathrm{Fe} \cos 2 \pi \cdot 3 \mathrm{p})^{2}$ \\
\hline 300 & st & $4(\mathrm{~S}+\mathrm{Fe})^{2}$ & 103 & $\mathrm{~m}-$ & $(\mathrm{S}-2 \mathrm{Fe} \cos 2 \pi \cdot 3 \mathrm{p})^{2}$ \\
\hline 001 & 0 & $4(\mathrm{~S}+\mathrm{Fe} \cos 2 \pi \cdot \mathrm{p})^{2}$ & $\begin{array}{l}113 \\
203\end{array}$ & $\begin{array}{c}0 \\
\operatorname{sch} w+\end{array}$ & $\begin{array}{l}4(\mathrm{~S}+2 \mathrm{Fe} \cos 2 \pi \cdot 3 \mathrm{p})^{2} \\
(\mathrm{~S}-2 \mathrm{Fe} \cos 2 \pi \cdot 3 \mathrm{p})^{2}\end{array}$ \\
\hline 101 & st & $(\mathrm{S}-2 \mathrm{Fe} \cos 2 \pi \cdot \mathrm{p})^{2}$ & 213 & st & $(s-2 F e \cos 2 \pi \cdot 3 p)^{2}$ \\
\hline 111 & 0 & $4(\mathrm{~S}+\mathrm{Fe} \cos 2 \pi \cdot \mathrm{p})^{2}$ & & & \\
\hline 201 & $\operatorname{sch} w+$ & $(\mathrm{S}-2 \mathrm{Fe} \cos 2 \pi \cdot \mathrm{p})^{2}$ & 004 & $\operatorname{sch} w+$ & $4(\mathrm{~S}+\mathrm{Fe} \cos 2 \pi \cdot 4 \mathrm{p})^{2}$ \\
\hline 211 & $\mathrm{~m}$ & $(\mathrm{~S}-2 \mathrm{Fe} \cos 2 \pi \cdot \mathrm{p})^{2}$ & 104 & $\operatorname{sch} w$ & $(\mathrm{~S}-2 \mathrm{Fe} \cos 2 \pi \quad 4 \mathrm{p})^{2}$ \\
\hline 301 & 0 & $4(S+F e \cos 2 \pi \cdot p)^{2}$ & 114 & st & $4(\mathrm{~S}+\mathrm{Fe} \cos 2 \pi \cdot 4 \mathrm{p})^{2}$ \\
\hline 002 & 0 & $4(\mathrm{~S}+\mathrm{Fe} \cos 2 \pi \cdot 2 \mathrm{p})^{2}$ & 005 & 0 & $4(\mathrm{~S}+\mathrm{Fe} \cos 2 \pi \cdot 5 p)^{2}$ \\
\hline 102 & st + & $(\mathrm{S}-2 \mathrm{Fe} \cos 2 \pi \cdot 2 \mathrm{p})$ & 105 & $\operatorname{sch} w$ & $(\mathrm{~S}-2 \mathrm{Fe} \cos 2 \pi \cdot 5 \mathrm{p})^{2}$ \\
\hline 112 & 0 & $4(\mathrm{~S}+\mathrm{Fe} \cos 2 \pi \cdot 2 \mathrm{p})^{2}$ & & & \\
\hline
\end{tabular}


Mit Rücksicht auf den Atomrauminhalt wäre es am natürlichsten, dass $p=\frac{1}{4}$ wäre, denn dann würde ein Fe-Atom auf beiden Seiten gleich weit von anderen Fe-Atomen sein. Aber mit $\mathrm{p}=\frac{1}{4}$ werden gewisse Strukturfaktoren zu gross, z. B. 001, während andere zu klein werden, z. B. 101.

Die folgenden Strukturtypen sind von $\mathrm{AMINOFF}^{1}$ diskutiert worden; sie werden jedoch hier der Vollständigkeit wegen kurz besprochen.

$\mathbf{D}_{3 \mathrm{~h}}^{\mathbf{4}}=\mathbf{D}_{6 \mathrm{~h}}^{\mathbf{4}} \quad$ 1) $=\mathrm{C}_{6 \mathrm{v}}^{\mathbf{4}}$ 2) für den Parameter $=\frac{1}{4}$. Wird in Zusammenhang mit dieser Struktur behandelt.

2) Diese Struktur passt ganz und gar nicht. So hat z. B. die Fläche 112 den Strukturfaktor $4(\mathrm{Fe}+\mathrm{S})^{2}$, also einen Maximalwert, aber auf dem Photogramm fehlt der Reflex.

3) Der Strukturfaktor wird derselbe für alle Flächen mit ungeradem L-Wert, wenn $\sin ^{2} 2 \pi \frac{\mathrm{H}-\mathrm{I}}{6}$ konstant gehalten wird.

So wird $\mathrm{A}^{2}$ dasselbe für $001,111,003,113,005$, nämlich gleich 0, was gut stimmt. Ferner dasselbe für 101, 103, 201, 203, 211, 213, nämlich $3 \cdot(\mathrm{Fe}-\mathrm{S})^{2}$. Dies steht nicht mit der beobachteten Intensität st für 101 in Übereinstimmung. 002, 112, 004 und 114 erhalten den Strukturfaktor $4(\mathrm{Fe}+\mathrm{S})^{2}$, der nur den Reflexen der beiden letzten Flächen entsprechen kann.

$\mathbf{C}_{\mathbf{6 r}}^{\mathbf{1}}$ Diese Struktur gibt der Fläche 001 den Strukturfaktor 4 $\left(\mathrm{Fe}^{2}+\mathrm{S}^{2}+2 \mathrm{FeS} \cos 2 \pi \mathrm{p}\right)$, der Fläche $101\left(\mathrm{Fe}^{2}+\mathrm{S}^{2}+2 \mathrm{FeS} \cos 2 \pi \mathrm{p}\right)$, der Fläche $0024\left(\mathrm{Fe}^{2}+\mathrm{S}^{2}+2 \mathrm{FeS} \cos 2 \pi .2 \mathrm{p}\right)$ und der Fläche 102 $\left(\mathrm{Fe}^{2}+\mathrm{S}^{2}+2 \mathrm{FeS} \cos 2 \pi \cdot 2 \mathrm{p}\right)$.

Hieraus geht hervor, dass für die Flächen 001 und 002 , die auf den Pulverphotogrammen fehlen, die Strukturfaktoren 4 mal so gross werden wie für 101 und 102, deren Reflexe st bezw. st+ sind.

$\mathbf{C}_{6 \mathbf{v}}^{\mathbf{4}}$ 1) In dieser Struktur erhält der Reflex 002 den Strukturfaktor $4\left(\mathrm{Fe}^{2}+\mathrm{S}^{2}+2 \mathrm{FeS} \cos 2 \pi .2 \mathrm{p}\right)$. Den gleichen Wert erhält $\mathrm{A}^{2}$ für die Fläche 112, während dagegen die Flächen 102, 202 und 212 nur ein viertel des grossen Strukturfaktors erhalten. Dies widerspricht durchaus den Beobachtungen, welche Schwärzung 0 für die zwei ersten und st + bezw. st für die anderen ergeben haben. Ausserdem werden einige vom Parameterwert unabhängigen Struk-

1 G. F. F. 44, 1922, 444 und Z. Krist. LVIII 1923, 203. 
Tab. 6.

\begin{tabular}{|c|c|c|c|c|c|}
\hline \multicolumn{6}{|r|}{$\left.\mathrm{C}_{6 v}^{4} 2\right)$} \\
\hline $\begin{array}{l}\text { In- } \\
\text { dices }\end{array}$ & $\begin{array}{l}\text { Sehwär- } \\
\text { zang }\end{array}$ & $\mathrm{A}^{2}$ & $\begin{array}{l}\text { In- } \\
\text { dices }\end{array}$ & $\begin{array}{c}\text { Schwär-- } \\
\text { zung }\end{array}$ & $A^{2}$ \\
\hline 001 & 0 & 0 & 100 & $\mathrm{~m}$ & $4(\mathrm{Fe}-\mathrm{S})^{2}$ \\
\hline 101 & st & $3 \mathrm{~S}^{2}$ & 110 & st & $4(\mathrm{Fe}+\mathrm{S})^{2}$ \\
\hline 003 & 0 & 0 & 200 & $\operatorname{sch} w$ & $4\left(\mathrm{Fe}-\frac{\mathrm{S}}{9}\right)^{2}$ \\
\hline 111 & 0 & 0 & 210 & $\operatorname{sch} w$ & $4\left(\mathrm{Fe}-\frac{\mathrm{S}}{\mathrm{a}}\right)^{2}$ \\
\hline 103 & $\mathrm{~m}-$ & $3 \mathrm{~S}^{2}$ & 300 & st & $4(\mathrm{Fe}+\mathrm{S})^{2}$ \\
\hline 201 & $\operatorname{sch} w+$ & $3 \mathbf{S}^{2}$ & & & \\
\hline 113 & 0 & 0 & 002 & 0 & $4\left(\mathrm{Fe}^{2}+\mathrm{S}^{2}+2 \mathrm{FeS} \cos 2 \pi \cdot 2 \mathrm{p}\right)$ \\
\hline 203 & sehw + & $3 S^{2}$ & 102 & st + & $4\left(\mathrm{Fe}^{2}+\frac{\mathrm{S}^{2}}{4}-\mathrm{FeS} \cos 2 \pi \cdot 2 \mathrm{p}\right)$ \\
\hline 005 & 0 & 0 & 112 & 0 & $4\left(\mathrm{Fe}^{2}+\mathrm{S}^{2}+2 \mathrm{FeS} \cos 2 \pi \cdot 2 \mathrm{p}\right)$ \\
\hline 211 & $\mathrm{~m}$ & $3 S^{2}$ & 004 & $\operatorname{sch} w+$ & $4\left(\mathrm{Fe}^{2}+\mathrm{S}^{2}+2 \mathrm{FeS} \cos 2 \pi \cdot 4 \mathrm{p}\right)$ \\
\hline 105 & $\operatorname{schw}$ & $3 S^{2}$ & 202 & st & $4\left(\mathrm{Fe}^{2}+\frac{\mathrm{S}^{2}}{4}-\mathrm{FeS} \cos 2 \pi \cdot 2 \mathrm{p}\right)$ \\
\hline 301 & 0 & 0 & 104 & $\operatorname{seh} w+$ & $4\left(\mathrm{Fe}^{2}+\mathrm{S}^{2}-2 \mathrm{FeS} \cos 2 \pi \cdot 4 \mathrm{p}\right)$ \\
\hline & & & $1: 4$ & st & $4\left(\mathrm{Fe}^{2}+\mathrm{S}^{2}+2 \mathrm{FeS} \cos 2 \pi \cdot 4 \mathrm{p}\right)$ \\
\hline & & & 212 & st & $4\left(\mathrm{Fe}^{2}+\frac{\mathrm{S}^{2}}{4}-\mathrm{FeS} \cos 2 \pi \cdot 2 \mathrm{p}\right)$ \\
\hline & & & 204 & & $4\left(\mathrm{Fe}^{2}+\frac{\mathrm{S}^{2}}{4}-\mathrm{FeS} \cos 2 \pi \cdot 4 \mathrm{p}\right)$ \\
\hline
\end{tabular}

turfaktoren $\mathrm{zu}$ gross. So wird $\mathrm{A}^{2}$ für 100, 200 und 210 gleich $(\mathrm{Fe}+\mathrm{S})^{2}$, also ein grosser Wert, während die geschätzten Intensitäten mittel und schwach sind.

$\mathbf{C}_{\mathbf{6 r}}^{\mathbf{4}} \quad$ 2) Für die $\mathrm{A}^{2}-\mathrm{Werte}$, in welchen der Parameter nicht einbegriffen ist, ist die Übereinstimmung gut, wie man sogleich aus Tab. 6 ersieht.

Damit der Strukturfaktor für die Flächen 002 und 112, die auf dem Pulverphotogramm keine Reflexe ergeben, so klein wie möglich werde, muss $\cos 2 \pi \cdot 2 p$ negativ und numerisch nahe zu 1 sein. Dann werden die $\mathrm{A}^{2}-$ Werte für 1()2, 202 und 212 gross, was mit den Beobachtungen stimmt. Damit der Strukturfaktor der Fläche 114 gross werden soll, muss auch $\cos 2 \pi \cdot 4 \mathrm{p}$ positiv und nahezu 1 werden. Diese Bedingung lässt sich gut mit dem Vorhergehenden vereinigen, und dadurch wird auch für die Flächen 104 und 204 die Übereinstimmung gut. Das Angeführte deutet auf einen Parameterwert von $\frac{1}{4}$, wodurch $\mathrm{C}_{6 \mathrm{v}}^{4}$ in $\mathrm{D}_{3 \mathrm{~h}}^{4}$ und $\mathrm{D}_{6 \mathrm{~h}}^{4}$ über- 
führt wird. Aber die Annahme dieses Wertes hat zur Folge, dass der Magnetkies in die höchste Symmetrieklasse versetzt wird. Versuche nach der Bestäubungsmethode von A. KundT haben keine Polarität in der Richtung der c-Achse gezeigt, was also die Richtigkeit des gewählten Parameterwerts bestätigt.

Aus Tabelle 7 und dem Diagramm Fig. 4 geht hervor, dass die Übereinstimmung zwischen geschätzten und berechneten Intensi-

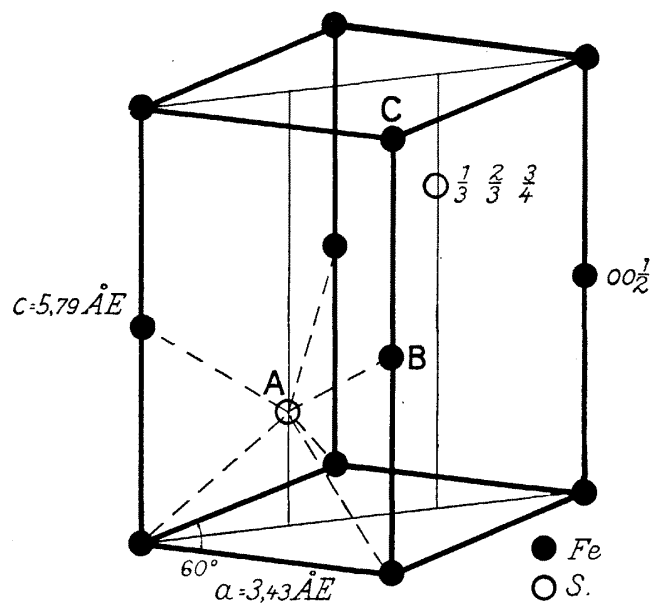

Fig. 2. Struktar des Magnetkieses. Anordnung der_Eisenatome um-ein Schwefelatom.

täten sehr gut ist, wenn die Fe-Atome die Koordinaten $000,00 \frac{1}{2}$ und die S-Atome $\frac{1}{3} \frac{2}{3} \frac{1}{4}, \frac{2}{3} \frac{1}{3} \frac{3}{4}$ haben. Als Mass für die Beugungsfähigkeit der Eisenatome ist die Atomnummer vermindert um $2\left(\mathrm{Fe}^{\cdot}\right)$ und für die S-A tome dieselbe vermehrt um $2\left(\mathrm{~S}^{\prime \prime}\right)$ genommen worden. Aber $\frac{\mathrm{Fe}^{*}}{\mathrm{~S}^{\prime \prime}}=\frac{24}{18}=\frac{4}{3}$, darum ist bei den Berechnungen Fe durch 4 und $\mathbf{S}$ durch 3 ersetzt worden.

Entschieden schlechter wird die Übereinstimmung, wenn man Fe und $\mathbf{S}$ den Platz wechseln lässt, so dass die S.Atome auf den hexagonalen Schraubenachsen placiert werden.

Wird $\mathrm{a}=3.43$ und $\mathrm{c}=5.79$ genommen, so wird der kürzeste Abstand zwischen den Fe- und den S-Atomen 2.45, was mit früher von BragG ${ }^{1}$ angegebenen Werten für die Atomradien für $\mathrm{Fe}$ und $\mathrm{S}$ in Übereinstimmung steht. Nach $\mathrm{B}$. ist ersterer $=1.40 \AA \mathrm{E}$ und letzterer 1.02, Summa 2.42.

1 Phil. Mag. 1920. 40, 169. 
Wie aus Fig. 2 ersichtlich, gruppiert ein Schwefelatom um sich in demselben Abstand 6 Eisenatome, die ein dreiseitiges Prisma mit der Kante des Dreiecks gleich a (3.43) und den Parallelkanten gleich $\frac{\mathrm{c}}{2}(2.90)$ bilden. Fig. 3 dagegen zeigt, dass die S-Atome, womit ein Fe-Atom umgeben ist, als Ecken in einem Oktaeder, das beinahe regulär ist, sich gruppieren; die Kanten sind nämlich abwechselnd 3.43 und 3.51 .

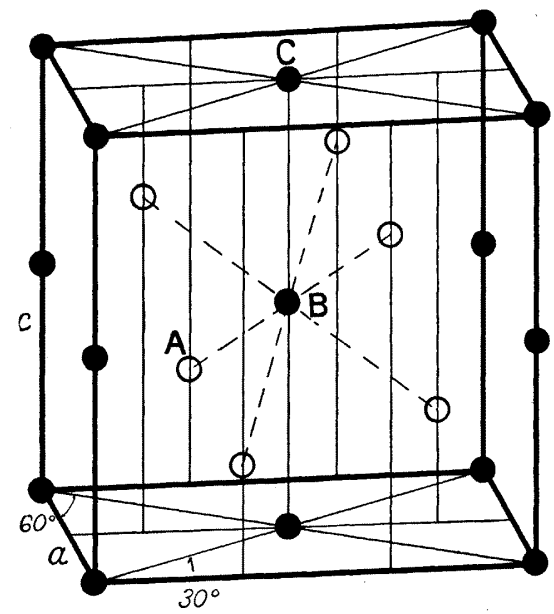

F g. 3. Anordnung der Schwefelatome um ein Eisenatom. Vergl. Fig. 2.

Anm. Das Vorhandensein des Reflexes 001 auf den Drehphotogrammen steht nicht in Übereinstimmung mit der oben hergeleiteten Struktur. Möglich ist, dass es sich bier um eine regelmässige Anordnung der überschüssigen S-Atome handelt. Eine solche Regelmässigkeit ist von G. Phragmén in einer festen Lösung von $14 \% \mathrm{Si}$ in Fe nachgewiesen worden.

\section{Breithauptit.}

Drehphotogramm. Ein kleines dünnes Kristallblatt von Andreasberg, mit $\mathrm{HCl}$ aus Calcit herausgelöst, ist in der Rotationscamera um eine an der Basis ausgebildete Kante gedreht worden. Im Photogramm erhält man den Abstand zwischen der 0- und 1-Schicht $=1.80 \mathrm{~cm}$, worauf a zu $3.90 \AA$. E. berechnet wird. Hieraus wird die eine Formelkonstante gleich 0.082 geschätzt. Die andere erhält man mit Leichtigkeit aus den für die Reflexe der 0-Schicht beobachteten $\sin ^{2} \frac{\hat{v}}{2}$ (Tab. 8), nämlich $=0.036$, und dieser Wert gibt $\mathrm{c}=5.09$, Die Werte für $\mathrm{c}$ und a geben das Achsenverbältnis 


\begin{tabular}{|c|c|c|c|c|c|c|c|c|c|c|c|c|c|c|c|}
\hline & $\mathbb{d}_{i}^{\infty}$ & 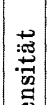 & $\begin{array}{l}\text { e. } \\
\text { d. } \\
\text { d. }\end{array}$ & 0 & $\begin{array}{l}1 \\
E \\
0 \\
0 \\
m\end{array}$ & 0 & $\vec{c}$ & 茂 & 0 & $\begin{array}{c}1 \\
+\infty \\
+\infty\end{array}$ & 0 & घ & 0 & 0 & $\begin{array}{l}+ \\
\vdots \\
\\
\end{array}$ \\
\hline & & 总 & 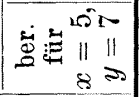 & 0 & $\widehat{\sigma}$ & $\stackrel{9}{\sim}$ & $\underset{\substack{0 \\
\mathfrak{0}}}{ }$ & 足 & 0 & ?̊ & 0 & है & 1 & $\mathscr{8}$ & 呆 \\
\hline & : & 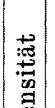 & 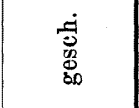 & 0 & 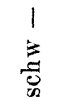 & $\underbrace{}_{0}$ & $\vec{b}$ & $\vec{\omega}$ & 0 & $+\infty$ & 0 & $\begin{array}{c}1 \\
⿱ 亠 䒑 ⿻ 心 ㇒\end{array}$ & 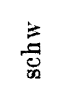 & घ & 0 \\
\hline & & 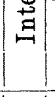 & 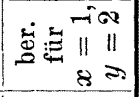 & 0 & 0 & $\ddot{\leftrightarrow}$ & 18 & 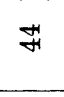 & 0 & 80 & 0 & $\overrightarrow{\mathrm{G}}$ & 0 & 0 & $\infty$ \\
\hline 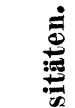 & జ & 茜 & $\begin{array}{l}\text { ij } \\
0 \\
0 \\
0\end{array}$ & 0 & $\vec{E}$ & 0 & +2 & $+\infty$ & 0 & 落 & 0 & $\underset{⿱ 亠 䒑}{1}$ & 0 & $\frac{\mathrm{E}}{\mathrm{d}}$ & 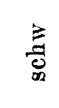 \\
\hline 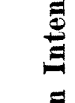 & & $\stackrel{\Xi}{g}$ & 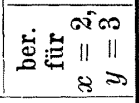 & 0 & $\infty$ & $\infty_{\infty}^{\infty}$ & 胫 & $\stackrel{90}{\longrightarrow 1}$ & 0 & $\underset{1}{8}$ & 0 & $\stackrel{\infty}{*}$ & $\stackrel{\infty}{\circ}$ & $\infty$ & $\stackrel{\mathfrak{M}}{\circledR}$ \\
\hline 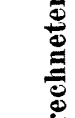 & 20 & & 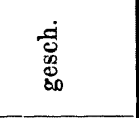 & 0 & $\exists$ & 0 & $\begin{array}{l}+ \\
\text { a }\end{array}$ & $\begin{array}{l}+ \\
\vec{b}\end{array}$ & 0 & $\overrightarrow{0}$ & 0 & $\Xi$ & $\begin{array}{l}1 \\
\vdots \\
0 \\
0 \\
0\end{array}$ & $\begin{array}{l}1 \\
0 \\
0 \\
0 \\
0\end{array}$ & $\begin{array}{l}+ \\
\vdots \\
0 \\
0\end{array}$ \\
\hline$\therefore \stackrel{\mathscr{E}}{\Xi}$ & $\sum_{i=}^{\infty}$ & & 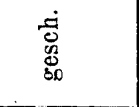 & 0 & $\exists$ & 0 & $\boldsymbol{g}$ & $\begin{array}{l}+ \\
+ \\
\dot{\omega}\end{array}$ & 0 & 落 & 0 & $\begin{array}{l}1 \\
\Xi\end{array}$ & $\begin{array}{l}1 \\
\text { a }\end{array}$ & 0 & घ \\
\hline Е & & 薄 & 总壳 & 0 & a & 0 & $\vec{n}$ & $\begin{array}{l}+ \\
+ \\
+\infty\end{array}$ & 0 & 崩 & 0 & $\frac{1}{a}$ & 总 & 0 & $\begin{array}{l}+ \\
\overrightarrow{0} \\
\text { d् }\end{array}$ \\
\hline $\begin{array}{l}5 \\
5 \\
5 \\
5\end{array}$ & 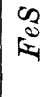 & 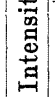 & 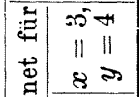 & 0 & $\stackrel{\circledast}{\sim}$ & की & 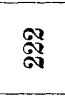 & $\underset{\sigma}{\stackrel{R}{R}}$ & 0 & $\stackrel{\mathscr{S}}{\stackrel{2}{2}}$ & 0 & 10 & $m$ & 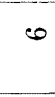 & $\mathscr{f}$ \\
\hline हू & & & 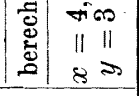 & 0 & $\stackrel{10}{R}$ & $\begin{array}{l}\infty \\
\infty\end{array}$ & 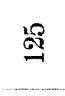 & 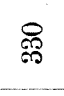 & 0 & $\stackrel{\mathscr{2}}{\stackrel{2}{二}}$ & 0 & $\stackrel{\infty}{\underset{*}{*}}$ & $\stackrel{9}{-1}$ & 0 & $\mathscr{f}$ \\
\hline 矛 & & & 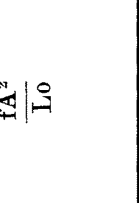 & 0 & $\begin{array}{c}\frac{\pi}{102} \\
\frac{8}{4} \\
\frac{\pi}{4}\end{array}$ & $\frac{1}{3}$ & مُ & 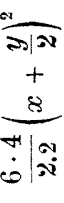 & 0 & $\begin{array}{l}\stackrel{a}{3} \\
+ \\
\frac{8}{\sigma} \\
+1\end{array}$ & 0 & 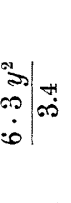 & $\begin{array}{c}x \cdot x \\
1 \\
8 \\
\infty\end{array}$ & 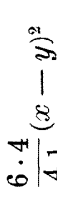 & $\begin{array}{c}\begin{array}{c}37 \\
5 \\
+ \\
8\end{array} \\
+18\end{array}$ \\
\hline & & & s. & $\overrightarrow{8}$ & 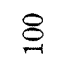 & 8 & $\vec{\Xi}$ & 今̊ & ஜे & $\stackrel{\varrho}{\exists}$ & $\exists$ & $\stackrel{9}{9}$ & §્. & $\stackrel{\vartheta}{二}$ & S' \\
\hline & & & 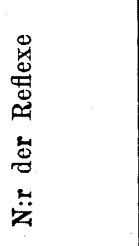 & $\begin{array}{l}\dot{ } \\
\dot{ } \\
\dot{ } \\
\dot{ } \\
.\end{array}$ & $\begin{array}{l}\cdot \\
\dot{ } \\
. \\
. \\
\text {. }\end{array}$ & $\begin{array}{l}\dot{ } \\
\dot{ } \\
\dot{ } \\
\dot{ } \\
\dot{ }\end{array}$ & $\begin{array}{l}\cdot \\
\dot{ } \\
. \\
\dot{H} \\
\dot{H}\end{array}$ & $\begin{array}{l}. \\
\dot{5} \\
\dot{ } \\
\dot{5} \\
\text { is }\end{array}$ & $\begin{array}{l}. \\
. \\
. \\
0\end{array}$ & $\begin{array}{l}. \\
. \\
. \\
. \\
. \\
.\end{array}$ & $\begin{array}{l}\dot{ } \\
\dot{ } \\
\dot{ } \\
\dot{ } \\
\dot{ }\end{array}$ & $\begin{array}{l}\dot{ } \\
\dot{ } \\
\dot{ } \\
\dot{0}\end{array}$ & $\begin{array}{l}\dot{ } \\
\dot{ } \\
\dot{ } \\
. \\
\circ\end{array}$ & . & . \\
\hline
\end{tabular}




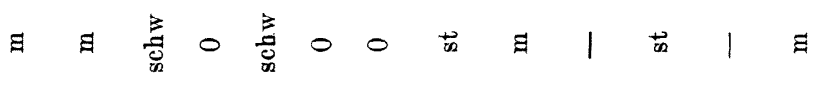

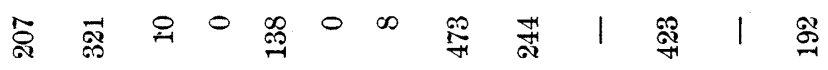

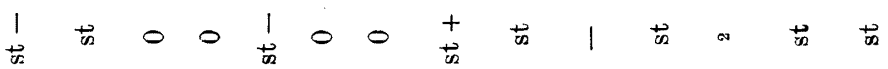

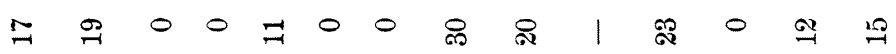

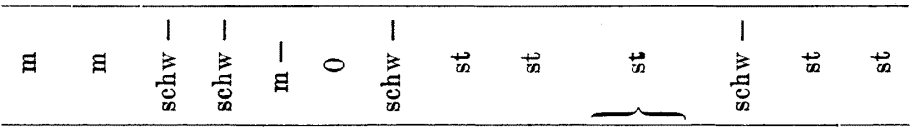

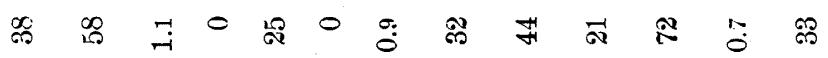

范总

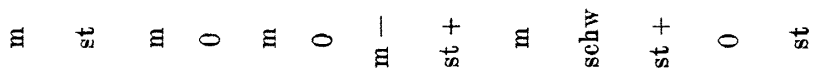

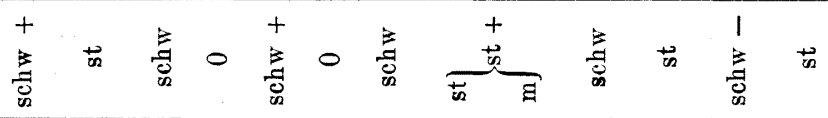

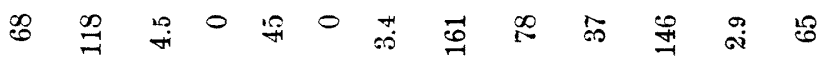

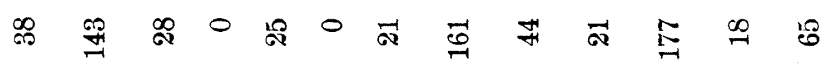

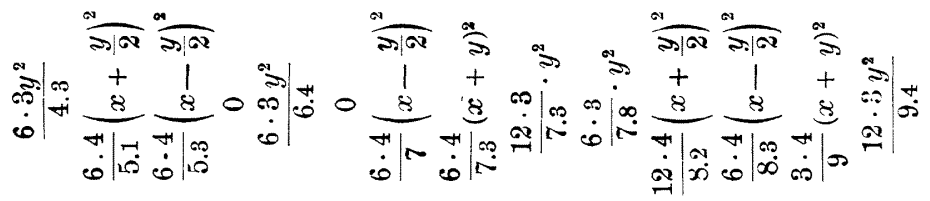

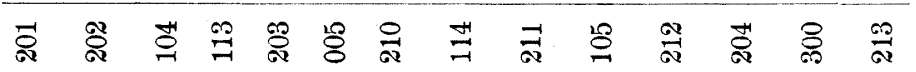

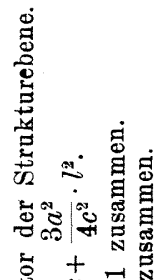

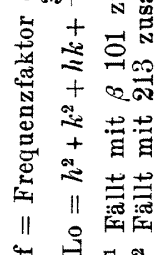

๓ ป 


\section{Berechnete}

Intensität

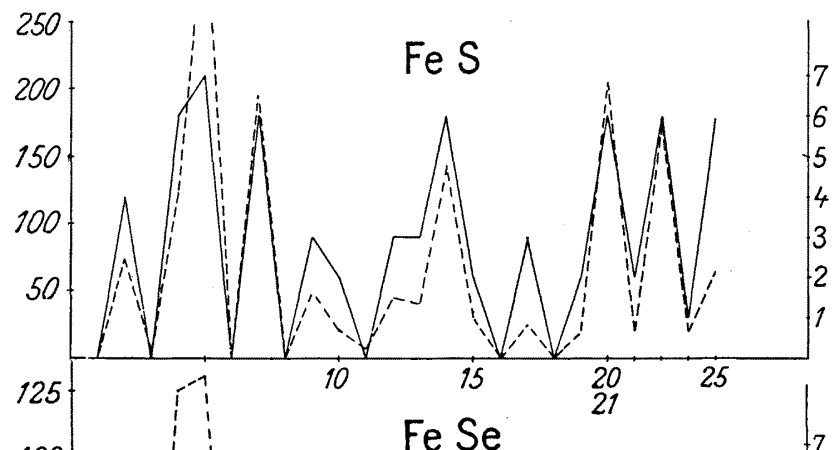

Geschätzte Intänsität
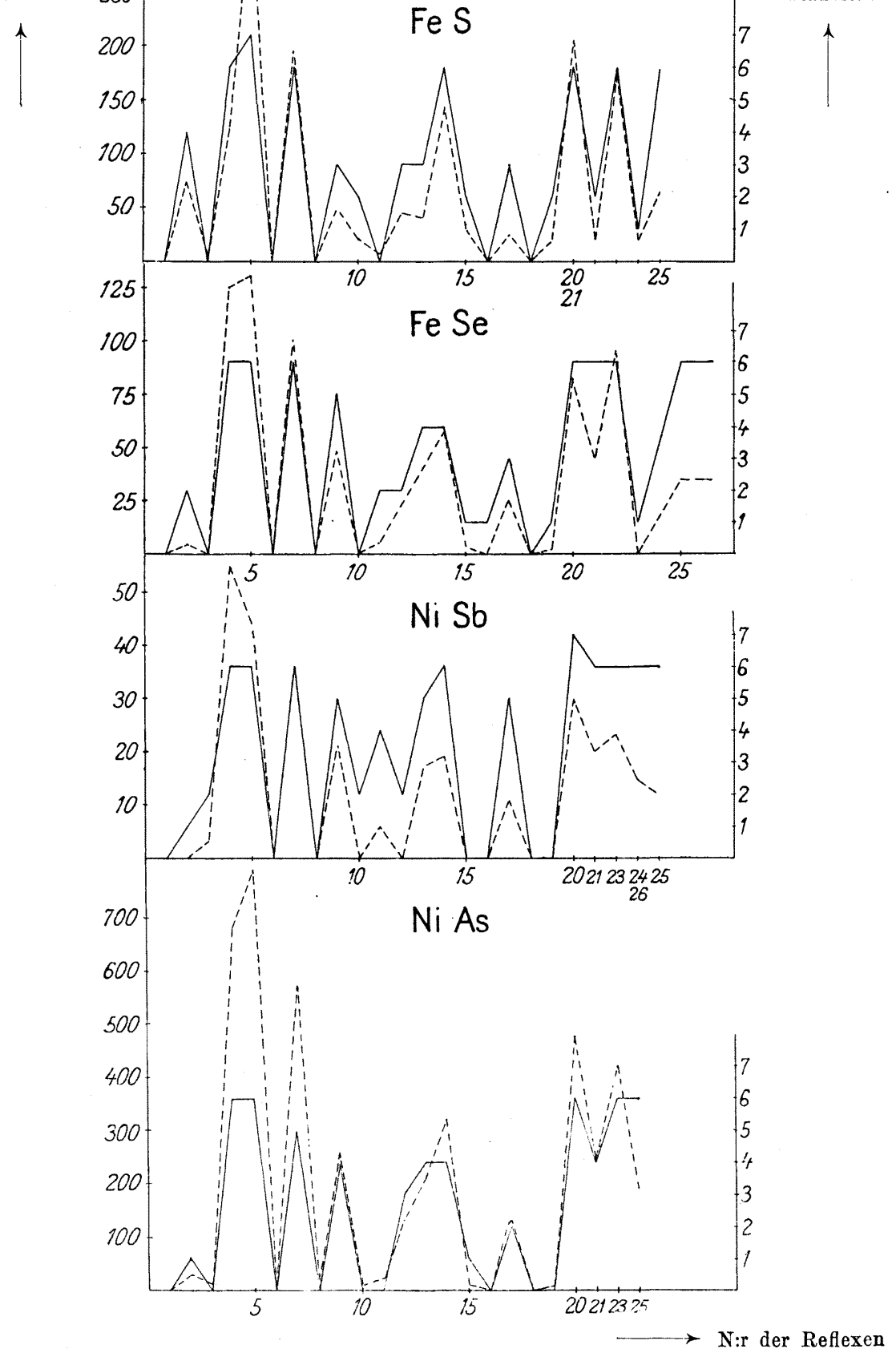

Fig. 4. Graphische Darstellang der Tab, Ұ. Statt schw - u. z, w. ist 1 bị 7 angewendet, 
Bd. 47. H. 1.] UNTERSUChUNG GEWISSER KRISTALlSTRUKTURIEN.

$=1.31$. Das von Busz ${ }^{1}$ an Andreasberger Kristallen morphologisch bestimmte Achsenverhältnis ist 1.294.

Vergleicht man die Reflexe auf dem Drehphotogramm mit denjenigen auf dem Pulverphotogramm, so findet man bemerkenswerte Unterschiede in der Intensität der Linien. So sind auf dem ersteren die Reflexe auf der Basis in der 2:ten und 4:ten Ordnung stark, während sie dagegen auf dem Pulverphotogramm schwach sind. Gerade umgekehrt verhält es sich mit den Reflexen von den Flächen 101 und 102, die in den Pulverphotogrammen stark, aber in den andern schwach sind.

Die Erklärung dieser Anomalien dürfte damit zusammenhängen, dass das Kristallblatt sehr dünn ist und dazu eine relativ grosse Basisfläche hat.

Lauephotogramm. Tafel 2 zeigt ein Lauephotogramm von Breithauptit mit der Strahlenrichtung senkrecht zur Basis und mit dem Abstand Kristall - Platte $=44.0 \mathrm{~mm}$. Die gnomonische Projektion davon (Fig. 5) zeigt grosse Ähnlichkeit mit sowohl derjenigen des Magnetkieses als auch der des Rotnickelkieses in der oben zitierten Arbeit von Aminoff (S. 215). Aus der gnomonischen

Tab. 8. Drehphotogramm von Breithauptit.

\begin{tabular}{|c|c|c|c|c|}
\hline \multirow[b]{3}{*}{ 0-Schicht } & \multirow[b]{3}{*}{. . . . . . } & \multicolumn{3}{|c|}{ Drehachse || einer Basiskaute } \\
\hline & & Indices & $\begin{array}{l}\text { Schwär- } \\
\text { zung }\end{array}$ & $\sin ^{2} \frac{9}{2}$ \\
\hline & & \begin{tabular}{|c|}
001 \\
101 \\
002 \\
102 \\
$\beta 103$ \\
201 \\
103 \\
$202, \beta 004$ \\
004 \\
203,104 \\
300 \\
$\beta 105$ \\
302 \\
105
\end{tabular} & $\begin{array}{l}\operatorname{sch} w \\
\operatorname{sch} w \\
m \\
\operatorname{sch} w \\
\operatorname{sch} w \\
\operatorname{sch} w \\
m \\
\mathrm{~m}- \\
\mathrm{st} \\
\mathrm{m}- \\
\operatorname{sch} w \\
\operatorname{sch} w \\
\operatorname{sch} w \\
\text { st }\end{array}$ & $\begin{array}{l}0.048 \\
0.120 \\
0.144 \\
0.231 \\
0.335 \\
0.371 \\
0.409 \\
0.473 \\
0.577 \\
0.651 \\
0.739 \\
0.799 \\
0.870 \\
0.965 \\
\end{array}$ \\
\hline 1-Schicht & . . . . . & $\begin{array}{l}102 \\
103 \\
212 \\
114 \\
213\end{array}$ & $\begin{array}{c}\text { st } \\
\text { st } \\
\text { schw } \\
\text { st } \\
\text { m }\end{array}$ & $\begin{array}{l}0.228 \\
0.409 \\
0.722 \\
0.811 \\
0.890\end{array}$ \\
\hline
\end{tabular}

${ }^{1}$ N. Jahrb, Min. etc. $1895,1,119$. 
Projektion wird das Achsenverhältnis $=1.32$ gemessen. Eine Schätzung der primitiven Translation parallel der c-Achse erhält man aus dem Abstand Zentralfleck - nächster Reflexpunkt. Dessen Wert $11.0 \mathrm{~mm}$ gibt der kleinste Glanzwinkel gleich 7.0 $0^{\circ}$ und $\mathrm{c} \sim 5,3$, wenn $\lambda_{\text {min. }}=0,16$ gesetzt wird. Aus den Dreh-und Lauephotogrammen geht hervor, dass die von Busz gewählten Achsen parallel mit denen des Elementarparallelepipeds sind.

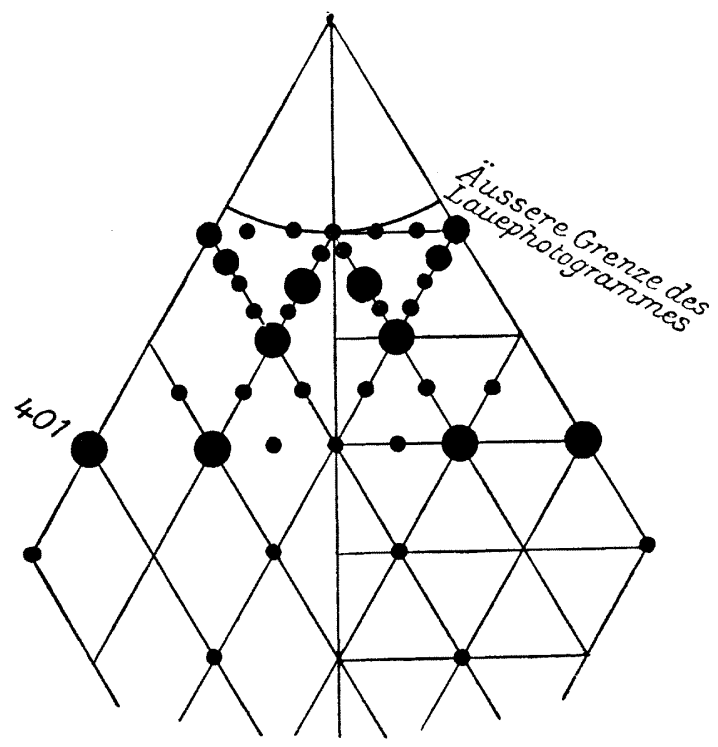

Fig. 5. Gnom. Proj. von Breithauptit anf Basis $(\mathrm{r} .=1 \mathrm{~cm})$.

Pulverphotogramme. Ausser von Andreasberger Kristallen sind Pulverphotogramme (Tab. 9) auch von einem aus $\mathrm{Ni}$ und Sb-Pulver synthetisierten Produkt genommen worden, dessen Photogramm grosse Übereinstimmung mit demjenigen des Minerals zeigt. Aus den $\sin ^{2} \frac{\boldsymbol{\vartheta}}{2}$-Werten werden die Formelkonstanten zu 0.0815 bezw. 0.0357 berechnet. Hieraus $\mathrm{a}=3.92, \mathrm{c}=5.11$ und $\frac{\mathrm{c}}{\mathrm{a}}=1.31$. Der Abstand zwischen zwei benachbarten $\mathrm{Ni}$ - und Sb-Atomen wird 2.59, wenn man die Struktur des Magnetkieses voraussetzt. Nach Braggs früherer Abschätzung $\mathrm{Ni}=1.35, \mathrm{Sb}=1.40$. S:a 2.75.

Unter der Annahme von zwei Molekülen im Elementargebiet wird das spez. Gewicht auf 8.73 berechnet. Eigene Bestimmungen des spezifischen Gewichtes haben niedrigere Werte ergeben, nämlich $8.37 ; 8.37 ; 8.46$; in Hrntzes Handbuch ist 7.5-8.4 angegeben. 
Bd 47. H. 1.] UNTERSUCHUNG GEWISSER KRISTALLSTRUKTUREN.

'Tab. 7 und Diagramm Fig. 4 zeigen, dass die Übereinstimmung zwischen den beobachteten und den berechneten Intensitäten eine gute ist, wenn man annimmt, dass der Strukturtyp des Magnetkieses auch für NiSb gilt.

\section{Rotnickelkies.}

Wie oben hervorgehoben wurde, hat AminofF ${ }^{1}$ die Kristallstruktur für Rotnickelkies bestimmt und zum ersten Mal den betreffenden Strukturtyp verwirklicht gefunden.

Tab. 9. Pulverphotogramm von Breithauptit.

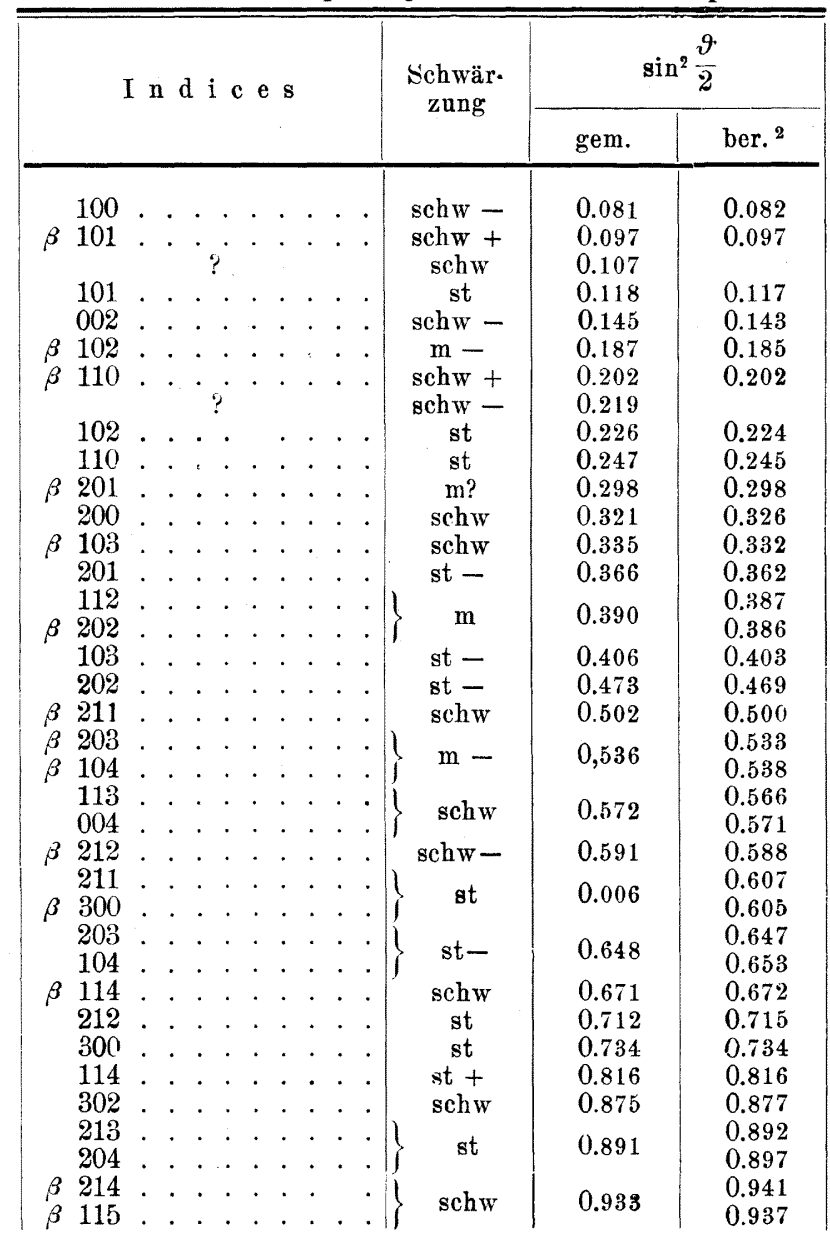

$\overline{1} \bar{Z}$. Krist. LVIII, 1923, 222.

${ }^{2}$ Formelkonstanten : $\mathrm{Fe}_{K \alpha}\left\{\begin{array}{l}0.0815 \\ 0.0357\end{array} \mathrm{Fe}_{K \beta}\left\{\begin{array}{l}0.0672 \\ 0.0294\end{array}\right.\right.$ 
Tab. 10, Präzisionsphotogramm von Rotnickelkies (NiAs) aus Eisleben.

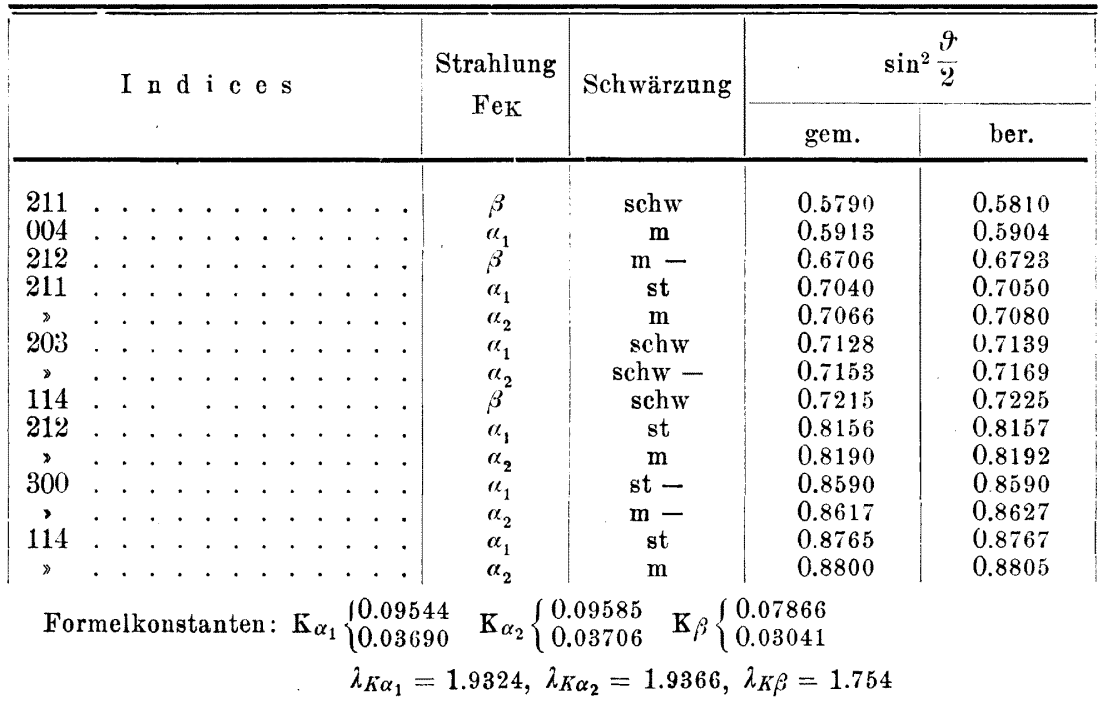

Vergleicht man indessen die Intensitäten der Linien des Pulverphotogrammes mit den berechneten Werten, so wird die Übereinstimmung für die zwei letzten Linien weniger gut. Aus diesem Grunde habe ich zwei Neuaufnahmen in einer gewöhnlichen Camera und in einer Präzisionscamera (Taf. 4) vorgenommen. Aus den erhaltenen Photogrammen, vor allem aus dem Präzisionsphotogramm (Tab. 10) geht deutlich hervor, dass die Indices der genannten Linien umgekehrt werden müssen. Siehe anch Tab. 7 und Fig. 4.

Die Konstanten der quadratischen Formel werden demgemäss für $\mathrm{K}_{\alpha}$ der Eisenstrahlung in 0.09544 und 0.03690 abgeändert. Hieraus wird $\mathrm{a}=3.610, \mathrm{c}=5.028$ und $\frac{\mathrm{c}}{\mathrm{a}}=1.393$, und der Abstand zwischen benachbarten Atomen entgegengesetzter Art $=2.434$ berechnet.

In einer eben publizierten Arbeit hat V. GoLdschmid ${ }^{1}$ teils die älteren Untersuchungen von Rotnickelkies einer kritischen Prüfung unterworfen, teils einige neue Messungen vorgenommen. Seine Resultate sind ungewöhnlich einstimmig; er berechnet für das Achsenverhältnis den Wert 1.3778 , der gut mit dem oben erhaltenen 1.39 stimmt. Von der Hemiedrie hat sich an ibren Kristallen nichts gezeigt, was für die gewählte Struktur noch eine Bestätigung mehr bedeutet.

1 Beiträge zar Kryst. und Min. Bd 2, Heft 2, 35. 


\section{Einige künstlich dargestellte Verbindungen mit derselben Struktur wie Magnetkies.}

Nis. Tab. 11 enthält eine Übersicht über gewisse NiS-Photogramme, die sich vollkommen analog denjenigen des Magnetkieses erweisen. Siehe auch Tafel 3 und 4. Aus den Formelkonstanten

Tab. 11. Pulverphotogramm von NiS.

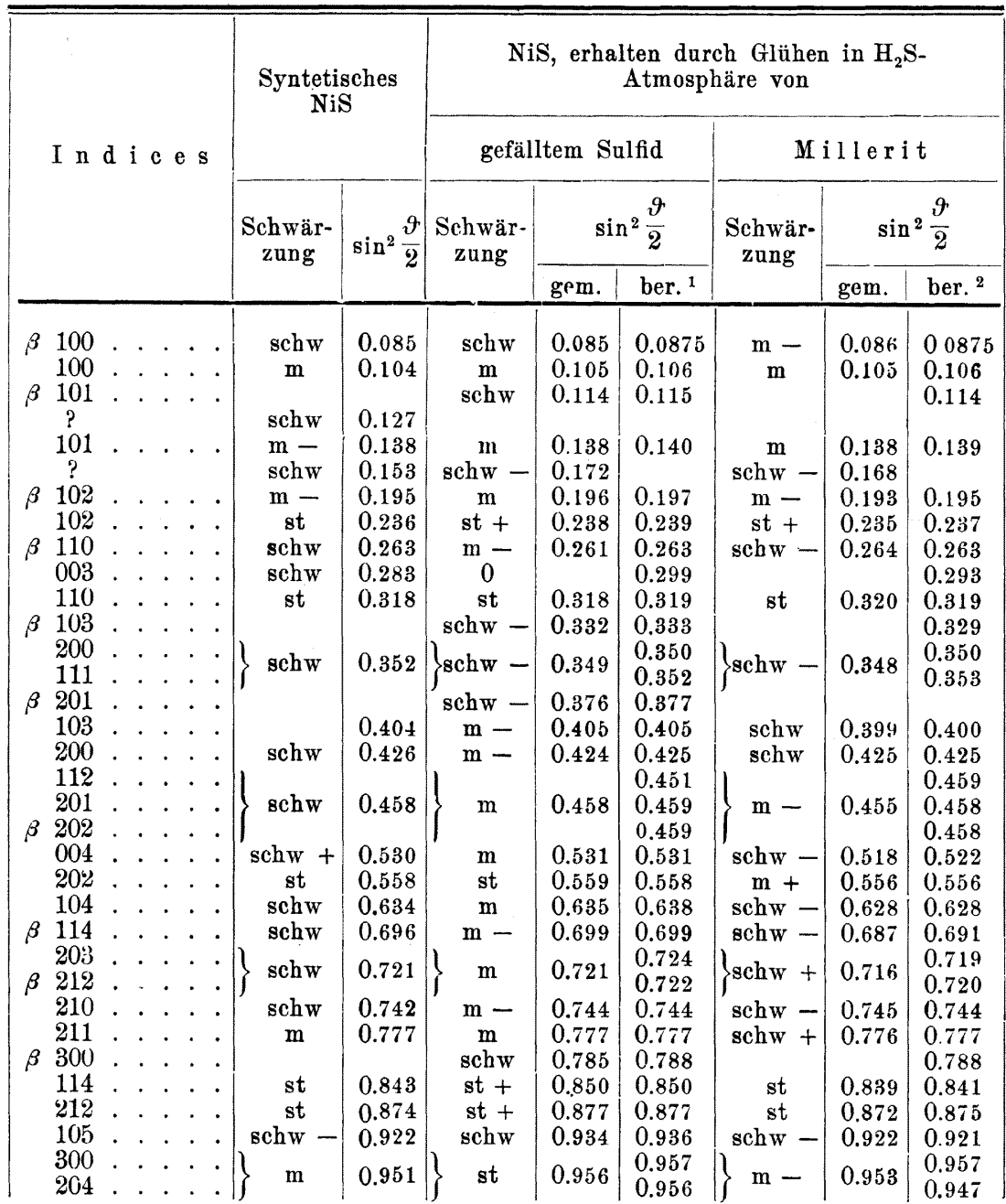

1 Formelkonstanten: $\mathrm{Fe}_{K \alpha}\left\{\begin{array}{l}0.1063 \\ 0.0332\end{array} \quad \mathrm{Fe}_{K} \beta\left\{\begin{array}{l}0.0875 \\ 0.0273\end{array}\right.\right.$

$2 \quad \therefore \quad\left\{\begin{array}{l}0.1063 \\ 0.0326\end{array} \gg\left\{\begin{array}{l}0.0875 \\ 0.0268\end{array}\right.\right.$ 
0.1063 und 0.0332 wird $\mathrm{a}=3.42, \mathrm{c}==5.30, \frac{\mathrm{c}}{\mathrm{a}}=1.55$ und der Abstand zwischen benachbarten Atomen verschiedener Art $=2.38$ berechnet. Hiermit werden die früheren Angaben Bragas betreffend die Atomradien verglichen: $\mathrm{Ni}=1.35$ und $\mathrm{S}=1.02$, also Summa $=2.37$.

CoS. Dass auch das CoS-Photogramm dem des Magnetkieses analog ist, geht aus Tafel 3 und Tab. 12 hervor. Die Formelkonstanten (für die $\mathrm{K}_{\alpha}$-Strahlung des Eisens) 0.1092 und 0.0354 ergeben $\mathrm{a}=3.37, \mathrm{c}=5.14, \frac{\mathrm{c}}{\mathrm{a}}=1.52$ und den Abstand zwischen be-

Tab. 12. Pulverphotogramm von CoS und (Fe, Co)S.

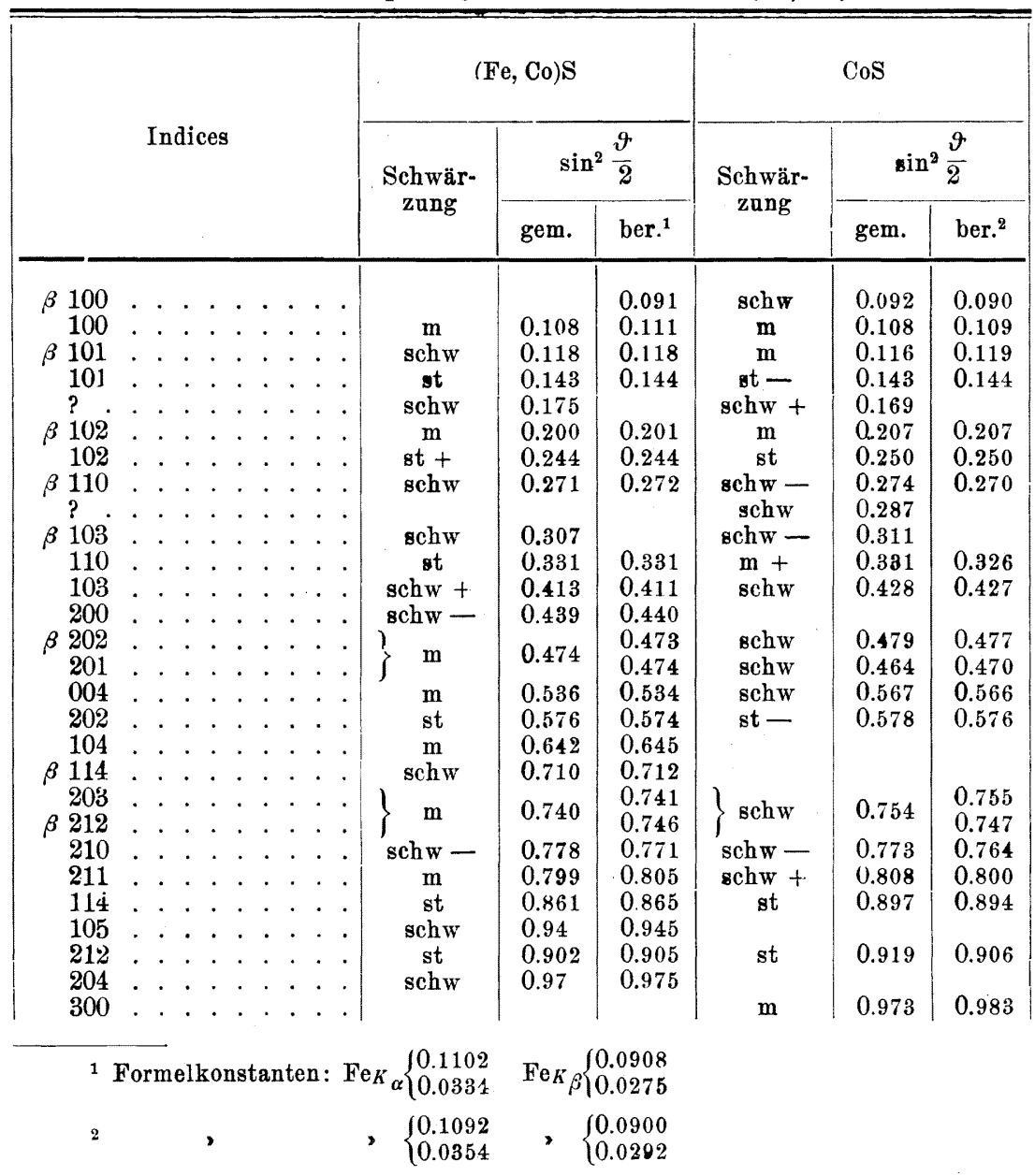


Bd 47. H. 1.] UNTERSUChUNG GeWisser Kristallstrukturen.

Tab. 13. Pulverphotogramm von (Ni, Fe)S.

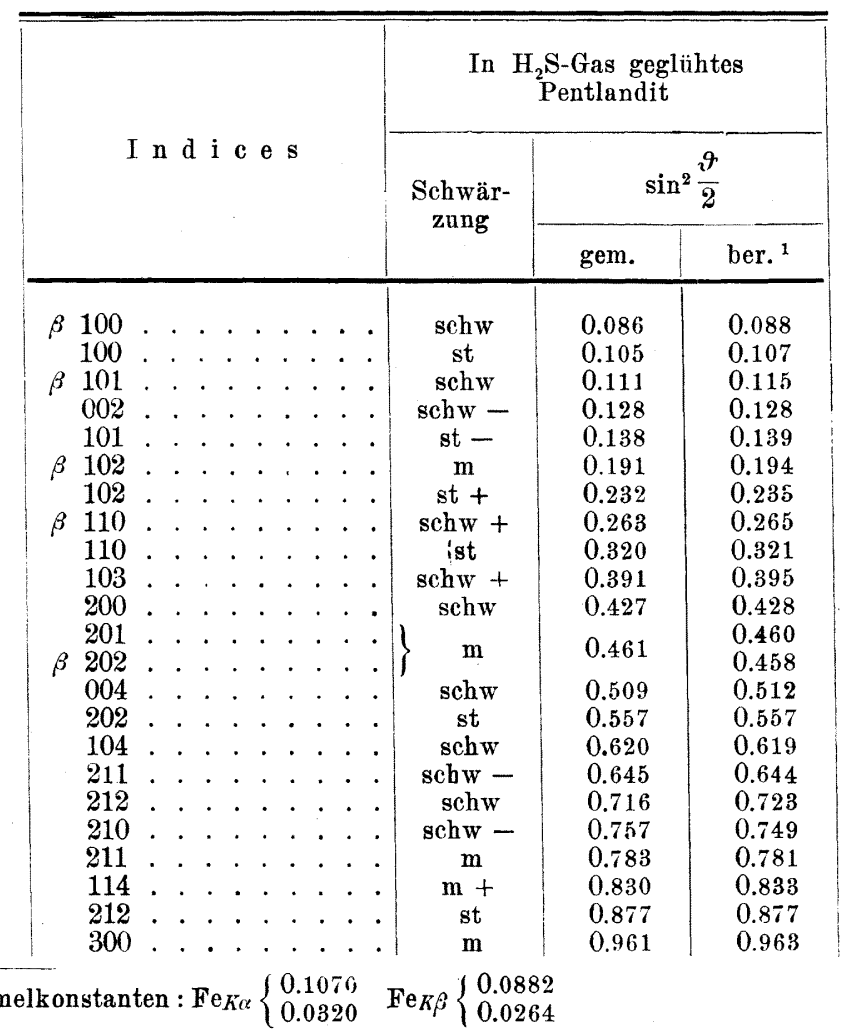

Tab. 14. Präzisionsphotogramm von $(\mathrm{Ni}, \mathrm{Fe}) \mathrm{S}$.

\begin{tabular}{|c|c|c|c|c|c|c|}
\hline \multicolumn{4}{|c|}{$\begin{array}{c}\text { Pentlandit } \\
\text { Unten auf der Platte }\end{array}$} & \multicolumn{3}{|c|}{$\begin{array}{l}\text { gefälltes Sulfid } \\
\text { Oben auf der Platte }\end{array}$} \\
\hline \multirow{2}{*}{ Indices } & \multirow{2}{*}{$\begin{array}{l}\text { Schwär- } \\
\text { zung }\end{array}$} & \multicolumn{2}{|c|}{$\operatorname{Sin}^{2} \frac{9}{2}$} & \multirow{2}{*}{$\begin{array}{l}\text { Schwär- } \\
\text { zang }\end{array}$} & \multicolumn{2}{|c|}{$\operatorname{Sin}^{2} \frac{9}{2}$} \\
\hline & & gemessen & berechnet & & gemessen & berechnet \\
\hline $\begin{array}{l}110 . \\
103 . \\
200 .\end{array}$ & $\begin{array}{c}\text { st } \\
\mathrm{m}- \\
\text { schw } \\
\mathrm{m}- \\
\mathrm{schw} \\
\mathrm{m}\end{array}$ & $\begin{array}{l}0.321 \\
0.380 \\
0.429 \\
0.457 \\
0.486 \\
0.548\end{array}$ & $\begin{array}{l}0.321 \\
0.381 \\
0428 \\
0.459 \\
0.486 \\
0.550\end{array}$ & $\begin{array}{c}\text { st } \\
\mathrm{m}- \\
\mathrm{sch} w \\
\mathrm{~m}- \\
\mathrm{sch} w \\
\mathrm{~m}\end{array}$ & $\begin{array}{l}0.322 \\
0.393 \\
0.429 \\
0.460 \\
0.506 \\
0.555\end{array}$ & $\begin{array}{l}0.321 \\
0.392 \\
0.428 \\
0.460 \\
0.506 \\
0.555\end{array}$ \\
\hline & melkonsta & $\mathrm{en}: \mathrm{Fe}_{\mathrm{K}_{\alpha}}$ & $\left\{\begin{array}{l}0.1071 \\
0.0304\end{array}\right.$ & & $\left\{\begin{array}{l}0.1071 \\
0.0316\end{array}\right.$ & \\
\hline
\end{tabular}

4-250333. G. F. F. 1925. 
nachbarten Co und S-Atomen $=2.33$. Dieser Wert wird mit dem aus den Atomradien nach BRAGG $\mathrm{Co}=1.37_{(\overline{5})}, \mathrm{S}=1.02_{(5)}$ berechneten Wert 2.40 verglichen.

(Fe, Ni)S. Das Photogramm des Mischkristalls ist denen der Komponenten ganz analog (Tab. 13). Tafel 4 zeigt eine Präzisions auf-

Tab. 15. Pulverphotogramm von FeSe und Fe (S, Se).

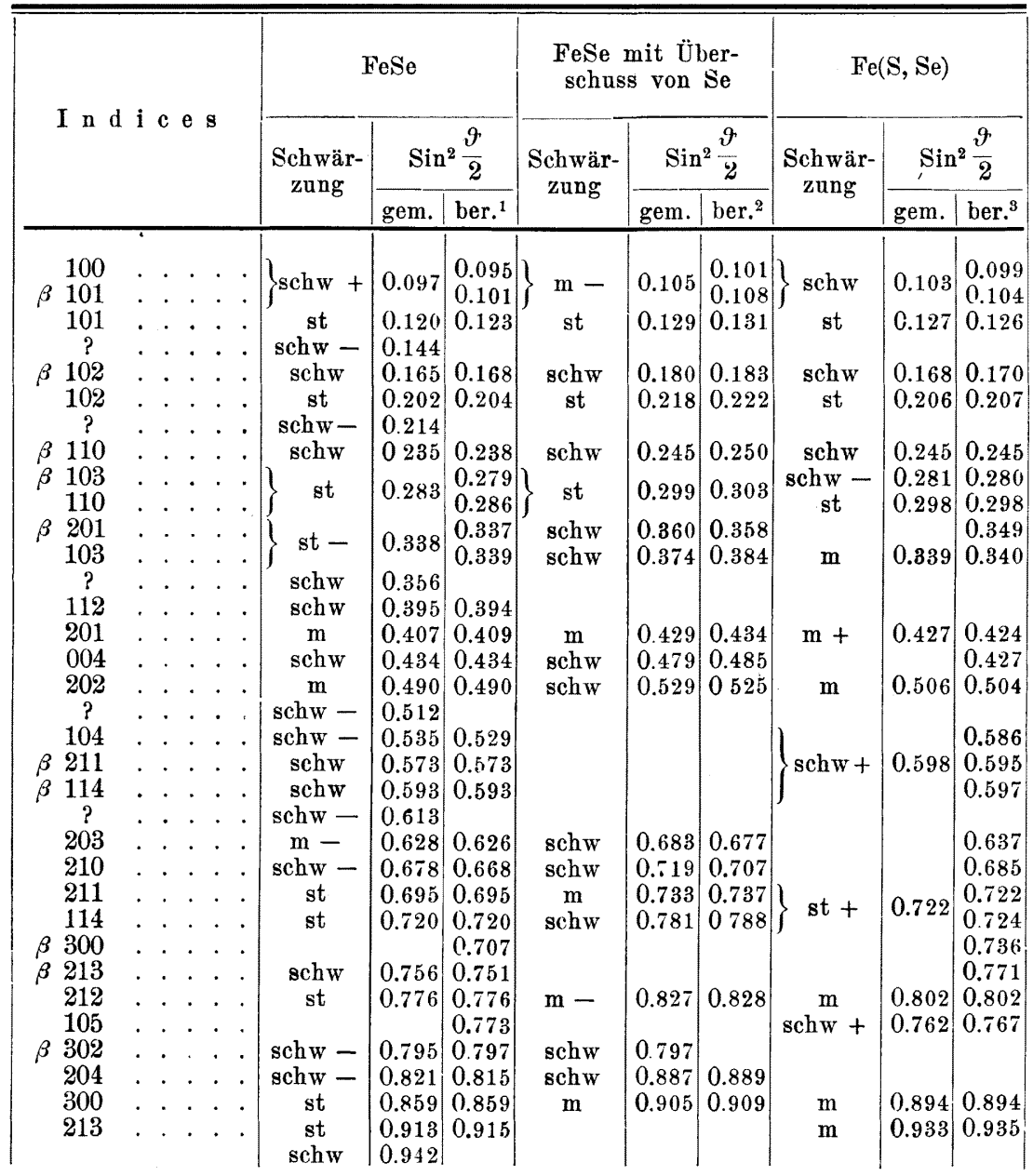

\footnotetext{
${ }^{1}$ Formelkonstanten: $\mathrm{Fe}_{K}\left\{\begin{array}{l}0.0954 \\ 0.0271\end{array} \mathrm{Fe}_{K}\left\{\begin{array}{l}0.0786 . \\ 0.0223\end{array}\right.\right.$

\begin{tabular}{|c|c|c|c|c|}
\hline 2 & 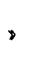 & \# & $\left\{\begin{array}{l}0.1010 \\
0.0303\end{array}\right.$ & , \\
\hline 8 & ' & 》 & $\left\{\begin{array}{l}0.0993 \\
0.0267\end{array}\right.$ & 》 \\
\hline
\end{tabular}
}


nahme von zwei auf verschiedene Weise erhaltenen Mischkristallen. Unten ist ein Photogramm von Pentlandit und oben von gefälltem Sulfid aufgenommen, welche beide in $\mathrm{H}_{2} \mathrm{~S}$-Gas geglüht sind.

Die Verschiebungen der Linien, die wahrscheinlich mit dem. S-Gehalt zusammenhängen. können direkt konstatiert werden. Dies kann auch aus Tab. 14 herausgelesen werden.

Die Formelkonstanten geben folgende Werte:

1) $\mathrm{a}=3.408, \mathrm{c}=5.540$ und $\frac{\mathrm{c}}{\mathrm{a}}=1.626$

2) $\mathrm{a}=3.408, \mathrm{c}=5.434$ und $\frac{\mathrm{c}}{\mathrm{a}}=1.594$

(Fe, Co)S. Fe und Co wahrscheinlich in stöchiometrischen Verhältnissen. Die Formelkonstanten sind hier 0.1102 und 0.0334, woraus $\operatorname{man} \mathrm{a}=3.36, \mathrm{c}=5.29$ und $\frac{\mathrm{c}}{\mathrm{a}}=1.57$ erhält. (Tab. 12).

Tab. 16. Pulverphotogramm von NiSe.

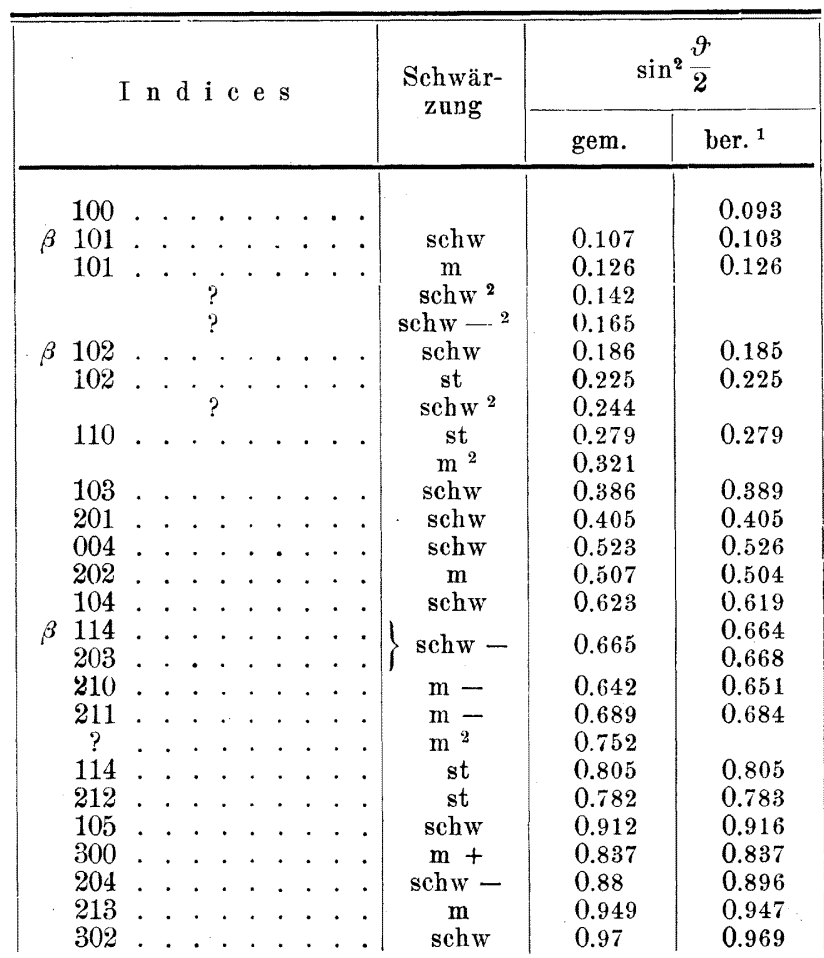

1 Formelkonstanten : $\mathrm{Fe}_{K \alpha}\left\{\begin{array}{l}0.0930 \\ 0.0329\end{array} \mathrm{Fe}_{K} \beta\left\{\begin{array}{l}0.0767 \\ 0.0271\end{array}\right.\right.$

2 Bei Vergleich mit anderen NiSe-Photogrammen zeigt sich, dass die Linie einer anderen Phase angehört. 
FeSe. Wie aus Tafel 3 und Tab. 15 hervorgeht, sind die Photogramme des Selenmagnetkieses denen des gewöhnlichen Magnetkieses sehr ähnlich. Von besonderem Interesse ist die grosse Variation der Gitterabstände, die die Zunahme des Selengehaltes zur Folge hat. Das in Wasserstoff geglühte Produkt, das also ohne nennenswerten Selenüberschuss ist, gibt Photogramme, deren $\sin ^{2} \frac{\theta}{2}$ die Formelkonstanten 0.0954 und 0.0271 , während dagegen das andere Produkt die Konstanten 0.1010 und 0.0303 erfordern. Hieraus wird im ersteren Falle $\mathrm{a}=3.61, \mathrm{c}=5.87, \frac{\mathrm{c}}{\mathrm{a}}=1,62$ und im letzteren Falle $\mathrm{a}=3.51, \mathrm{c}=5.55, \frac{\mathrm{c}}{\mathrm{a}}=1.58$ berechnet. Im ersteren Falle erhält man den kürzesten Abstand zwischen den Selen- und den Eisenatomen $=2.55$. Der aus den Braggschen Atomradien $\mathrm{Fe}=1.40$ und $\mathrm{Se}=1.17$ berechnete Wert ist 2.57 .

Fe (S, Se). Tab. 15 gibt die Formelkonstanten 0.0993 und 0.0267, woraus $\mathrm{a}=3.54, \mathrm{c}=5.91$ und $\frac{\mathrm{c}}{\mathrm{a}}=1.67$ berechnet wird.

Tab. 17. Pulverphotogramm von $\mathrm{Ni}_{3} \mathrm{~S}_{2}$.

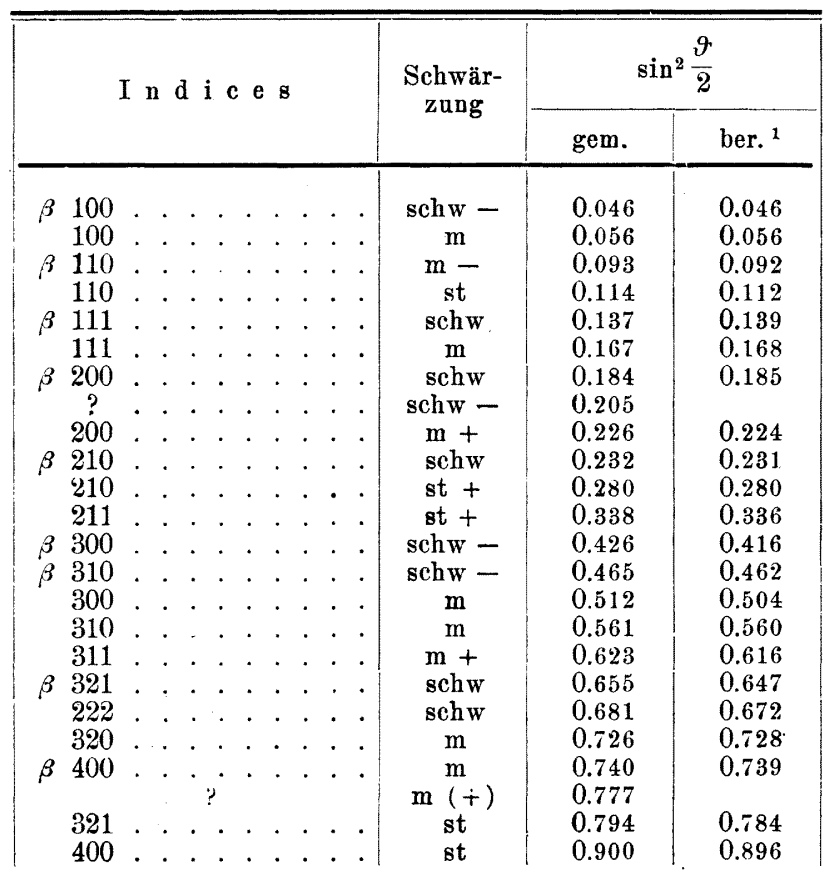

1 Formelkonstanten : $\mathrm{Fe}_{K \alpha} 0.0560, \mathrm{Fe}_{K} \beta 0.0462$ 
Ni Se. Die Substanz, deren Photogramm in Tab. 16 aufgenommen ist, ist offenbar kein reines NiSe. Durch Vergleichen von Photogrammen verschiedener NiSe-Produkte haben gewisse Linien wegen ihrer wechselnden Intensität als nicht zur Hauptphase gehörig ausgeschaltet werden können. Aus den Formelkonstanten 0.0930 und 0.0329 wird $\mathrm{a}=3.66, \mathrm{c}=5.33, \frac{\mathrm{c}}{\mathrm{a}}=1.46$ und der kürzeste Abstand zwischen den $\mathrm{Ni}$ - und den Se-Atomen $=2.50$ berechnet. Die von Bragg angegebenen Werte der Atomradien 1.35 bezw. 1.17 ergeben 2.52.

$\mathbf{N i}_{3} \mathbf{S}_{2}$. Weil das durch Synthese dargestellte NiS nie völlig rein ist, hat Verf. versucht, das Produkt durch Schmelzen homogen zu machen. Von der erstarrten Masse metallischen Aussehens hat Verf. Pulverphoto. gramme (Tab. 17) aufgenommen. Sie sind von denen des NiS ganz verschieden: Die $\sin ^{2} \frac{\vartheta}{2}$ können als Multipla von 0.056 geschrieben werden, weshalb man auf ein kubisches Elementarparallelepiped schliessen kann. Aus der Konstante 0.056 wird die Kante des Elementarkubus zu 4.08 berechnet. Aus der Gleichung $\mathrm{a}^{3}=1.65$. M. N, wo $\mathrm{s}$ das spez. Gewicht $=5.85$ (eigene Bestimmung) ist, erhält man $\mathrm{N} . \mathrm{M}=238$, was mit $\mathrm{N}=1$ und der Formel $\mathrm{Ni}_{3} \mathrm{~S}_{2}$ für die Substanz stimmt.

Eine Analyse hat den S-Gehalt $26.4 \%$ ergeben. Die Formel $\mathrm{Ni}_{3} \mathrm{~S}_{2}$ bedingt $26.7 \% \mathrm{~S}$.

Später hörte Verf. von den Untersuchungen K. BonNemanns ${ }^{1}$, die gezeigt haben, dass beim Zusammenschmelzen von Schwefel und Nickel die Verbindung $\mathrm{Ni}_{3} \mathrm{~S}_{2}$ statt $\mathrm{NiS}$ erhalten wird.

Verf. beabsichtigt nicht, in diesem Zusammenhang eine Bestimmung von der Struktur des $\mathrm{Ni}_{3} \mathrm{~S}_{2}$ zu geben. Dazu dürften zweifelsohne entweder Laue- oder Drehphotogramme erforderlich sein.

\section{Gültigkeitsbereich der Kristallstruktur Typus Rotnickelkies.}

Die Wahrscheinlichkeit scheint dafür zu sprechen, dass dieser Strukturtyp durch die Mehrzahl zweiatomiger Verbindungen zwischen $\mathrm{Fe}, \mathrm{Ni}$ oder $\mathrm{Co}$ einerseits und einem andern Grundstoff anderseits verwirklicht wird. $\mathrm{NiO}$, das regulär kristallisiert und eine Struktur von NaCl-Typ hat, bildet hiervon eine Ausnahme. Indessen scheint, soweit man jetzt weiss, jener Strukturtyp auf Verbindungen der Metalle $\mathrm{Fe}, \mathrm{Ni}$ und Co beschränkt zu sein. Die benachbarten Elemente im periodischen System $\mathrm{Mn}, \mathrm{Cu}$ und $\mathrm{Zn}$ haben auch zweiatomig zusammengesetzte Sulfide. Von diesen sind $\mathrm{MnS}$, Alabandin, und ZnS, Zinkblende bezw. Wurtzit röntgeno-

1 Metallurgie 5, 13 u. 61 . 
graphisch untersucht, und keines derselben ist nach dem Typ des Rotnickelkieses gebaut. Über die Verbindung CuS hat Verf. eine röntgenographische Untersuchung begonnen, die dargetan hat, dass $\mathrm{CuS}$ eine bedeutend kompliziertere Struktur als FeS hat.

Das Lauephotogramm, senkrecht auf einem Spaltblatt von Covellin aufgenommen, zeigt volle hexagonale Symmetrie. Aus dem Abstand der innersten Flecken bis zum Zentralfleck erhält man eine Schätzung des Identitätsabstandes in der Strahlenrichtung $=9.0 \AA$. E.

Ein Drehphotogramm von dem für das Lauephotogramm angewendeten Spaltblatt ist noch nicht aufgenommen worden. Statt dessen wurde ein anderer Kristall benutzt und die Rotation erfolgte um eine der vielen parallelen Kanten, die offenbar BasisKanten sind (Tafel 1). Aus dem Abstand 1 Schichtlinie-1 Schichtlinie wird der Identitätsabstand in der Richtung der Rotationsachse $=6.69$ berechnet, der Abstand 2 Schichtlinie-0 Schichtlinie ergibt 6.63, und aus der 3 Schichtlinie erhält man 6.66.

Pulver-Photogramme sind sowohl von Covellin als auch von künstlich dargestelltem $\mathrm{CuS}$ aufgenommen worden. Die Photogramme sind unter einander identisch, aber sie zeigen eine augenscheinliche Verschiedenheit von denjenigen des Magnetkieses. Covellin kann demnach eine Kristallstruktur von jenem Typ nicht haben. Es liegt also eine gewisse Wahrscheinlichkeit dafür vor, dass dieser Strukturtyp auf $\mathrm{Fe}-$, Ni- und $\mathrm{Co}^{-V}$ Verbindungen beschränkt ist.

\section{Pentlandit.}

Drehphotogramme. Aus einer Stufe aus Beiern, enthaltend Pentlandit mit deutlicher oktaedrischer Spaltbarkeit, wurde ein kleines Bruchstück herausgesucht und in einer Drehcamera mit der Drehungsachse parallel mit einer Oktaederkante photographiert. Bei Messung des Abstandes zwischen den 1-Schichtlinien erhält man den Identitätsabstand parallel mit der Oktaederkante $b_{1}=7.42$. Wird der Abstand zwischen der 0- und 2-Schichtlinie benutzt, so erhält man $b_{2}=7.06$ und bei Anwendung des Abstandes 0-1Schichtlinie $b_{3}=7.19$. Mittel: $7.22 \AA$. E.

Wie aus den erhaltenen Werten für $b$, wo die Übereinstimmung weniger gut ist, ersichtlich, ist die Drehungsachse nicht exakt parallel mit der Oktaederkante gewesen. Daher ist ein anderes Drehphotogramm und diesmal mit der Drehungsachse parallel mit 
Bd 47. H. 1.] UNTERSUCHUNG GEWISSER KRISTALLSTRUKTUREN.

der Kubuskante aufgenommen worden (Tafel 1), wobei die Einstellung mit Hilfe eines Lauephotogrammes bewerkstelligt wurde. ${ }^{1}$

Die aus dem Abstand der 1-, 2- und 3-Schichtlinie von der 0Schichtlinie erhaltenen Werte für die Identitätsabstände a längs der Kubuskante sind $=10.08,9.97$ und $9.96 \AA$ A. E. Mittel $=10.00$ A. E.

Hieraus die Konstante $=0.0093$ in der quadratischen Formel für kubische Kristalle: $\sin ^{2} \frac{\vartheta}{2}=$ Konst. $\left(\mathrm{H}^{2}+\mathrm{K}^{2}+\mathrm{L}^{2}\right)$.

Aus dem ersten Drehphotogramm wird $b=7.22 \AA$. E. und aus dem zweiten $\mathrm{a}=10.00$ berechnet. Zwischen diesen gilt, dass $\mathrm{b} \sqrt{\overline{2}}=\mathrm{a}$ ist. Dies setzt voraus, dass das Gitter flächenzentriert ist, da für ein einfaches Gitter die Bedingung $a \sqrt{2}=b$ ist.

Lauephotogramme. Wie bereits erwähnt, ist das Photogramm (Tafel 2) mit der Strahlenrichtung parallel mit der Kubuskante aufgenommen. Es zeigt höchste kubische Symmetrie. Das Photogramm ist in Fig. 6 zu gnomonischer Projektion übergeführt. Es kommen keine gemischten Indices vor ausser 411, die in zweiter Ordnung spiegelt, ein weiterer Beweis dafür, dass das Gitter flächenzentriert ist. Eine Schätzung der Kubuskante erhält man aus dem Abstand des innersten Flecks zum Zentrum. Dieser beträgt $1.02 \mathrm{~cm}$. Da der Abstand der Platte vom Kristall $4.85 \mathrm{~cm}$ ist, erhält man den kleinsten Glanzwinkel gleich $5^{\circ} .9$, und a wird approximativ $=8.5$

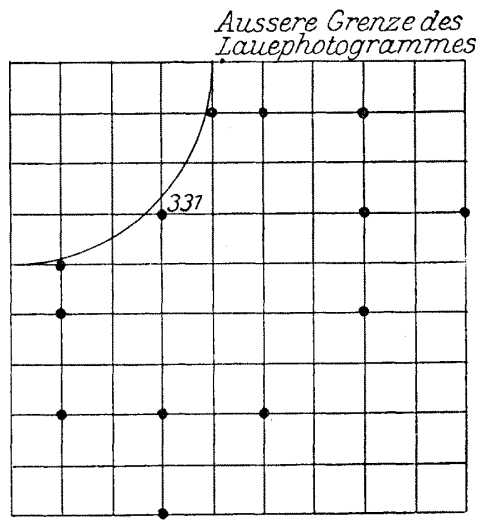

Fig. 6. Gnom. Proj. von Pentlandit auf einer Kubusfläche. berechnet, wenn $\lambda \min =0.18$ gesetzt wird.

Pulverphotogramme. Anfangs sind Photogramme von Pentlandit aus Sudbury genommen worden. Später wurde teils Pentlandit aus Beiern und teils synthetisch dargestelltes Material mit wechselnden Proportionen $\mathrm{Fe}: \mathrm{Ni}$ angewendet. Die einzelnen Photogramme (Tab. 18) sind so gut wie identisch. Die Formelkonstante

1 Jette, Phragmén, Westgren: Journal of the Institute of Metals. Vol. XXXI, 1, $1924,195$. 
für das Photogramm von Pentlandit aus Beiern ist jedoch ungefähr $1 \%$ höher als für die anderen Substanzen.

Aus der Konstante 0.0931 wird die Kubuskante $a=10.01$ berechnet und aus der andern Konstante 0.00921 erhält man $\mathrm{a}=$ 10.06 .

Anzah1 Moleküle im Elementarkubus. Aus den beiden Ausdrücken für das Volumen des Elementarkubus ergibt sich die Anzahl der Moleküle gleich 33, wenn man das spez. Gewicht gleich 4.9 setzt. In Hintzes Handbuch ist 4.60-5.01 angegeben. Eigene Bestimmung an synthetischem Produkt 4.93.

33-zählige Lagen gibt es indessen nicht im kubischen System, wohl aber 32-zählige. Die nächst höhere Zahl ist 48 und die niedrigere 24, die nicht in Frage kommen können. Wird das spez. Gewicht für das Pentlandit aus der Annahme berechnet, dass $\mathrm{N}=32$ ist, so erhält man $\mathrm{s}=4.7$, was in nur annähernder Übereinstimmung mit den soeben zitierten Ziffern steht.

Bestimmung der Struktur. In der folgenden Erörterung der Strukturen werden alle S-Atome gleichwertig angenommen. Für die $\mathrm{Ni}$ - und Fe-Atome wird zuerst die Annahme gemacht, dass sie alle gleichwertig sind, dann dass sie zwei ungleichwertige Lagen einnehmen. Im letzteren Falle wird vorausgesetzt, dass $\mathrm{Ni}$ - und Fe-Atome in weitem Masse einander substituieren können.

$\mathrm{Da}$ das Lauephotogramm vierzählige Symmetrie zeigt, muss man alle den hemiedrischen Lauephotogrammen entsprechenden Raumgruppen ausschliessen. Aus den beiden Drehphotogrammen kann sicher herausgelesen werden, dass das Gitter flächenzentriert sein muss. In Übereinstimmung hiermit fallen Reflexe von allen Flächen mit gemischten Indices weg.

Die flächenzentrierten kubischen Raumgruppen, welche holoedrischen Lauephotogrammen entsprechen, sind folgende: $\mathrm{T}_{\mathrm{d}}^{2} \mathrm{~T}_{\mathrm{d}}^{5} 0^{3} 0^{4}$ $0_{\mathrm{h}}^{5} 0_{\mathrm{h}}^{6} 0_{\mathrm{h}}^{7}$ und $0_{\mathrm{h}}^{8}$.

Von diesen werden $\mathrm{T}_{\mathrm{d}}^{5}, 0_{\mathrm{h}}^{6}$ und $0_{\mathrm{h}}^{8}$ ausgeschlossen, für welche Reflexe von Flächen ( $\mathrm{h} \mathrm{k} \mathrm{l}$ ), wo $\mathrm{h} \mathrm{k}$ und $\mathrm{l}$ ungerade sind und $\mathrm{h}=\mathrm{k}$ ist, ${ }^{1}$ fehlen sollen. Auf den Filmen kommen nämlich 113, $331,333,115,335,117$ u. s. w. vor.

Schliesslich gestattet $\mathrm{T}_{d}^{2}$ keine 32-zähligen Lagen, und demnach verbleiben nur die Raumgruppen $0^{3}, 0^{4}, 0_{\mathrm{h}}^{5}$ und $0_{\mathrm{h}}^{7}$.

1 R. W. G. Wyскоғн: Am. Journal of Science IV 1922. 184. 
Bd 47. H. 1.] UN'TERSUCHUNG GEWISSER KRISTALLSTRUKTUREN.

Tab. 18. Pulverphotogramm von Pentlandit.

\begin{tabular}{|c|c|c|c|c|c|c|c|c|c|c|}
\hline \multirow{3}{*}{\multicolumn{2}{|c|}{ Indices }} & \multicolumn{3}{|c|}{$\begin{array}{c}\text { Pentlandit ans } \\
\text { Beiern }\end{array}$} & \multicolumn{2}{|c|}{ P. ans Sudbury } & \multicolumn{2}{|c|}{$\begin{array}{c}\text { Synthetischer } \\
\text { P. zu gleichen } \\
\text { Teilen Fe und } \\
\text { Ni }\end{array}$} & \multicolumn{2}{|c|}{$\begin{array}{l}\text { Synth. P. mit } \\
\text { doppelter } \\
\text { Menge Ni }\end{array}$} \\
\hline & & \multirow{2}{*}{$\begin{array}{c}\text { Schwär- } \\
\text { zung }\end{array}$} & \multicolumn{2}{|c|}{$\sin ^{2} \frac{\vartheta}{2}$} & \multirow{2}{*}{$\begin{array}{c}\text { Schwär- } \\
\text { zung }\end{array}$} & \multirow{2}{*}{$\begin{array}{l}\sin ^{2} \frac{9}{2} \\
\text { gem. }\end{array}$} & \multirow{2}{*}{$\begin{array}{c}\text { Schwär- } \\
\text { zung }\end{array}$} & \multirow{2}{*}{\begin{tabular}{|l|}
$\sin ^{3} \frac{\vartheta}{2}$ \\
gem.
\end{tabular}} & \multirow{2}{*}{$\begin{array}{l}\text { Schwär- } \\
\text { zang }\end{array}$} & \multirow{2}{*}{$\begin{array}{l}\sin ^{2} \frac{9}{2} \\
\text { gem. }\end{array}$} \\
\hline & & & gem. & ber. ${ }^{1}$ & & & & & & \\
\hline 111 & - & $\operatorname{ch} w$ & 027 & 0.0279 & & & & & & \\
\hline 220 & 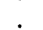 & & & $0: 0745$ & & & & & & \\
\hline$\beta \mathbf{3 1 1}$ & . & schw & 83 & 0.0844 & sehw & 0.080 & $\operatorname{sch} w-$ & 0.080 & $\operatorname{sch} w$ & 0.08 \\
\hline$\beta \quad 222$ & $\dot{.}$ & $\operatorname{sch} w-$ & 91 & 0.0920 & & & & & & \\
\hline 311 & . & st & 1 & 0.102 & st & 0.100 & at & 0.099 & st & 0.09 \\
\hline 222 & . & $\mathrm{m}$ & & 0.112 & $\operatorname{sch} w$ & 0.108 & $\operatorname{seh} w+$ & 0.109 & & .10 \\
\hline 400 & . & schw & 0 & 0.149 & $\operatorname{sch} w-$ & 0.144 & $\operatorname{sch} w-$ & 0.144 & $\operatorname{seh} w$ & 0.14 \\
\hline 331 & . & $\mathrm{m}$ & 0 & 0.177 & $\operatorname{sch} w$ & 0.171 & lw & 0.171 & & 0.1 \\
\hline $\begin{array}{l}\beta \\
\beta \\
\beta\end{array}$ & $\cdot$ & $\operatorname{schw}$ & 0.205 & 0.207 & $\operatorname{seh} w$ & 0.204 & $\operatorname{sch} w-$ & 0.206 & $\operatorname{sch} w$ & 0.2 \\
\hline 422 & $\dot{.}$ & $\operatorname{sch} w$ & 0.220 & 0.223 & schw - & 0.220 & & & $\operatorname{schw}$ & 0.2 \\
\hline$\beta 440$ & . & & & 0245 & $\mathbf{m}$ & 0.239 & & & & \\
\hline $\begin{array}{l}511 \\
333\end{array}$ & $\dot{.}$ & st & 0.248 & 0.251 & $\mathrm{~m}$ & 0.245 & st, breit & 0.245 & st & 0.2 \\
\hline $\begin{array}{ll}\beta & 600 \\
\beta & 442\end{array}$ & $\cdot$ & $\operatorname{sch} w$ & 0.271 & 0.276 & & & & & $\operatorname{sch} w$ & \\
\hline 440 & $\dot{.}$ & st + & 0.298 & 0.298 & st + & 0.291 & st + & 0.295 & st + & 0.29 \\
\hline 531 & . & $\mathrm{m}$ & 0.331 & 0.326 & $\operatorname{sch} w-$ & 0.321 & \} $\operatorname{seh} w-$ & 0.318 & $\mathrm{~m}$, breit & 0.33 \\
\hline 620 & $\dot{.}$ & $\operatorname{sch} w+$ & 0.371 & $\left|\begin{array}{l}0.336 \\
0.372\end{array}\right|$ & $\begin{array}{l}\operatorname{sen} \\
\operatorname{seh}\end{array}$ & $\begin{array}{l}0.331 \\
0.369\end{array}$ & & & & \\
\hline 533 & $\cdot$ & $m$ & 0.402 & 0.400 & $\operatorname{seh} w$ & 0.395 & schw, & 0.399 & $\mathrm{~m}$, breit & 0.40 \\
\hline 622 & . & $\mathrm{m}$ & 0.409 & 0.409 & $\operatorname{schw}$ & 0.403 & & & & \\
\hline в 731 & $\cdot$ & $\mathrm{m}-$ & 0.452 & 0.454 & $\operatorname{sch} w$ & 0.445 & $\operatorname{seh} w$ & 0.445 & & \\
\hline 711 & : & $m_{-}$ & 0476 & 0474 & & 0468 & & & & \\
\hline 551 & . & $\mathrm{m}-$ & 0.46 & 0.484 & - & 0.468 & & & & \\
\hline $\begin{array}{r}640 \\
\beta 800\end{array}$ & $\cdot$ & $\operatorname{sch} w$ & 0.491 & $\begin{array}{l}0.484 \\
0.491\end{array} \mid$ & $3 \operatorname{sch} w-$ & 0.486 & & & & \\
\hline$\beta 733 .$. & . & $\operatorname{sch} w$ & 0.516 & 0.514 & $\operatorname{sch} w--$ & 0.514 & & & & \\
\hline $\begin{array}{l}731 \\
553\end{array}$ & · & \} & 0.552 & 0.549 & $\mathrm{~m}$ & 0.543 & $\mathbf{m}$ & 0.542 & $\mathbf{m}+$ & 0.5 \\
\hline 800 & - & st & 0.597 & 0.596 & $\ldots$ & 0.592 & & 0.589 & & 0.59 \\
\hline & & $\mathrm{m}$ & 0.625 & 0.624 & $\operatorname{sch} w$ & 0,616 & $\operatorname{sch}$ & 0.619 & $\operatorname{sch} w$ & 0.6 \\
\hline$\beta \begin{array}{l}751,555 \\
931 .\end{array}$ & 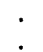 & st & 0.697 & \begin{tabular}{|c|}
0.698 \\
0.698
\end{tabular} \mid & $\mathrm{m}$ & 0.693 & $\operatorname{sch} w+$ & 0.690 & $\mathrm{~m}$ & 0.69 \\
\hline 662 & . & $\mathrm{m}-$ & 0.706 & 0.707 & & & & & & \\
\hline$\beta 844$. & & $\mathrm{m}$ & 0.735 & 0.734 & schw & 0.725 & schw & 0.726 & $\mathbf{m}$ & 0.727 \\
\hline $\begin{array}{l}91 \\
75\end{array}$ & : & $\mathrm{m}$ & 0.774 & 0.773 & $\operatorname{sch} w-$ & 0.76 & schw - - & 0.760 & $\operatorname{sch} w$ & 0.79 \\
\hline & & $\operatorname{sch} w$ & 0.8 & 0.819 & & & & & & \\
\hline & & st & 0.848 & 0.847 & m & 0.843 & $\operatorname{sch} w+$ & 0.835 & m & 0.83 \\
\hline 84 & & st + & 0.894 & 0.894 & st & 0.885 & st & 0.882 & st & 0.88 \\
\hline 771 & & $\mathrm{~m}$ & 0.92 & $|0.921|$ & m & 0.920 & schw - & 0.91 & & \\
\hline
\end{tabular}

1 Formelkonstanten: $\mathrm{Fe}_{K \alpha} 0.00931, \mathrm{Fe} K \beta \quad 0.00767$. 
$0^{4}$ und $0_{\mathrm{h}}^{7} \quad$ Für die Raumgruppen $0^{4}$ und $0_{\mathrm{h}}^{7}$ gibt es zwei verschiedene 16-zählige Lagen und ausserdem 32-zählige mit einem Freiheitsgrad.

Struktur I: $16 \mathrm{~b}, 16 \mathrm{c}$ und $32 \mathrm{~b} .^{1}$

Dies stimmt recht gut, wenn $p$ nahezu $=1 / 4$ ist. Dieser $p$-Wert ist indessen unmöglich, denn dann würden $4 \mathrm{~S}$-Atome sich beinahe auf einen Punkt zusammendrängen. Sieht man ein Modell an, so findet man, dass $p$, mit Rücksicht auf den Atomrauminbalt, ungefähr $3 / 8$ sein muss. Aber in solchem Fall erhält der Strukturfaktor für 400 einen grossen Wert, während dagegen die Linie auf dem Film schwach ist.

So verbleibt die Möglichkeit, $p$ einen Wert zwischen $1 / 4$ und $3 / 8$, beispielsweise gerade dazwischen, also $\mathrm{p}=\frac{5}{16}$, zu geben. Auch hier scheint die Übereinstimmung zwischen beobachteten und berechneten Werten weniger gut zu werden. Ausserdem ist diese Struktur wegen ungleichmässiger Atomverteilung unwahrscheinlich.

Struktur II: 32b mit zwei verschiedenen Werten für den Parameter. Als wahrscheinlichste sind $\frac{1}{16}$ und $\frac{5}{16}$ gewählt. Die Atomanordnung, die innerhalb gewisser Gebiete sehr eng ist, wird in anderen besonders spatiös. Daher ist die Struktur II in hohem Grade unwahrscheinlich und dürfte bei der Diskussion ausser Betracht gelassen werden. Ausserdem ist diese und folgende Struktur mit gleichwertigen $\mathrm{Fe}$ - und Ni-Atomen weniger wahrscheinlich, da beim Schmelzen der einfachen Verbindungen Pentlandit nicht entsteht.

Struktur III: 32 a mit den Parametern $\frac{1}{16}$ und $\frac{3}{16}$, als den wahrscheinlichsten.

Diese Struktur erklärt das Nichtvorhandensein folgender Reflexe, für welche der Strukturfaktor 0 wird, nämlich: 200, 420, 442, 444, $640,642,820,644,840$ und 842. Dagegen erhalten die Strukturfaktoren für die Reflexe 822 und 660, die auf den Filmen fehlen, einen grossen Wert. Die Reflexe von den Flächen 440 und 844 sind st+, aber die Strukturfaktoren für dieselben werden 0. Struktur III muss also verworfen werden.

$\mathbf{0}^{3}$ und $\mathbf{0}_{\mathrm{h}}^{\mathbf{5}}$ Innerhalb der Raumgruppen $0^{3}$ und $0_{\mathrm{h}}^{5}$ gibt es teils 8-zählige, teils 24-zählige Lagen zweier Arten und endlich 32zählige Lagen mit 1 Freiheitsgrad.

${ }^{1}$ R. W. G. Wyckows:The analytical expression of the theory of spacegroups. 
Bd 47. H. 1.] UNTERSUCHUNG GEWISSER KRISTALLSTRUKTUREN.

Struktur IV: Fe: 8-zählige Lagen

$\mathrm{Ni}$ : 24-zählige Lagen

S: 32-zählige Lagen

Damit der Abstand zwischen $\mathrm{S}$ und allen den 4 benachbarten Ni-bezw. Fe-Atomen derselbe werde, muss $p^{1 / 8}$ sein, was im folgenden zuerst angenommen wird.

Der Strukturvorschlag IV hat einige grosse Differenzen zur Folge. Auf den Photogrammen fehlen die Reflexe 200, 420, 442, $640,820,644,842$, aber nach IV würde der Strukturfaktor gross werden. Dieser Vorschlag muss also verworfen werden.

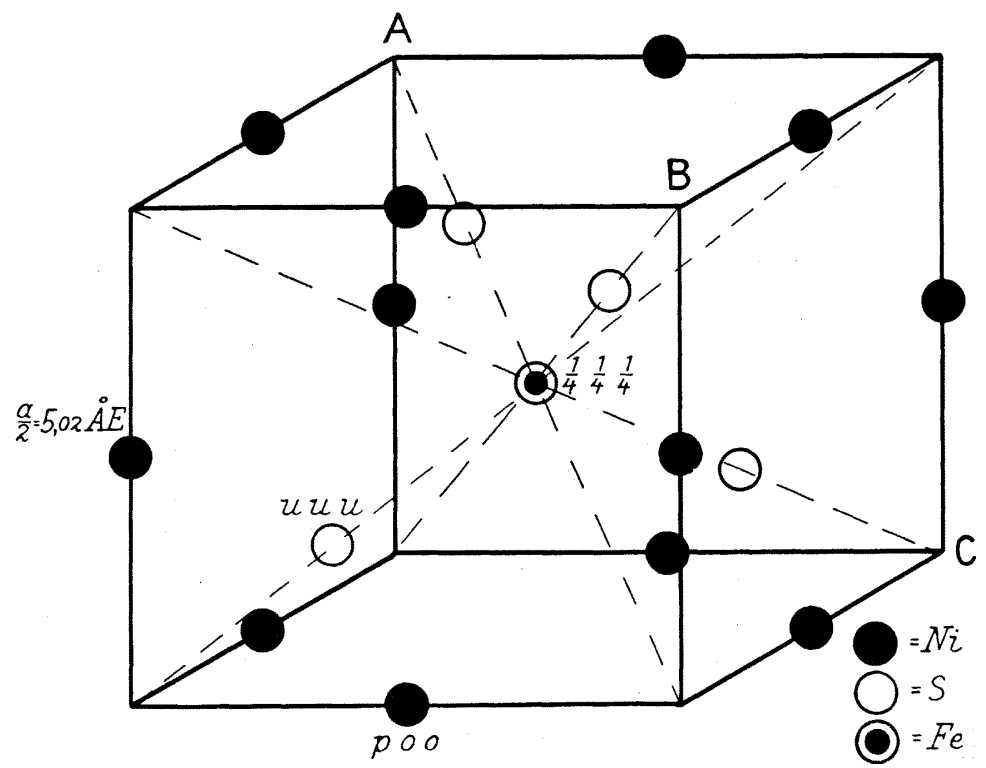

Fig. 7. Struktur des Pentlandits. Ein Viertel des Elementarkubus.

Versucht man es mit anderen Werten für $p$, die kleiner als $1 / 8$ sind, wodurch der Abstand $\mathrm{Ni}-\mathrm{S}$ und $\mathrm{Fe}-\mathrm{S}$ mehr in Übereinstimmung mit früher erhaltenen Resultaten kommt, werden einige der Strukturfaktoren bessere Werte erhalten, aber andere schlechtere, und auch diese Möglichkeiten müssen verworfen werden.

Der Abstand $\mathrm{Ni}-\mathrm{Fe}$ wird, für alle $\mathrm{p}$-Werten gleich $\frac{\mathrm{a}}{4}$, d.h. 2.50 und $\mathrm{Ni}-\mathrm{Ni}=3.55=\frac{\mathrm{a}}{4} \sqrt{2}$. Der Abstand $\mathrm{S}-\mathrm{S}=2.20=2 \cdot 0.11 \cdot 10.0$, wenn der Parameter 0.11 ist. Unter der gleichen Voraussetzung wird $\mathrm{Ni}-\mathrm{S}=2.26$ und $\mathrm{Fe}-\mathrm{S}=2.42$. 
Den Parameterwert grösser als $\frac{1}{8}$ zu nehmen, dürfte nicht in Frage kommen, denn das würde eine Verminderung des Abstandes $\mathrm{Fe}-\mathrm{S}$ unter $2.17 \AA$ A. E. besagen.

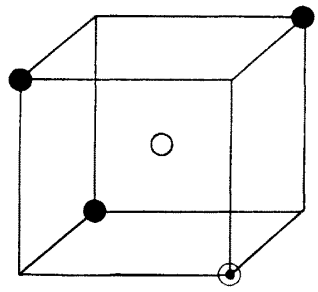

a

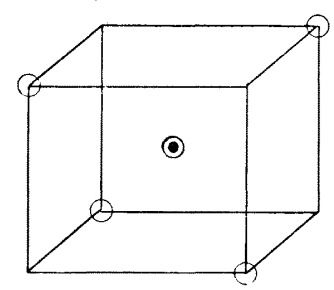

b

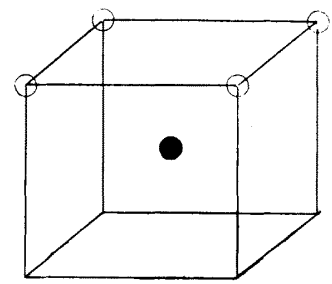

c

Fig. 8. Anordnung der Atome in der Pentlanditstruktur.

Struktur V: 8 e und 32 a wie zuvor aber 24 a anstatt 24 c. (Fig. 7. Der ganze Elementarkubus wird durch Spiegelung in den Ebenen $\mathrm{AB}, \mathrm{AC}$ und $\mathrm{BC}$ erhalten.) Der Parameter in 24 a wird $=\frac{1}{4}$ und in $32 \mathrm{a}=\frac{1}{8}$ gewählt. Aus den beigefügten Tab. 19 und Fig. 9 geht hervor, dass die Übereinstimmung im ganzen ziemlich gut ist. So fehlen Reflexe für alle Flächen mit gemischten Indices und die übrigen fehlenden Reflexe entsprechen Flächen mit kleinen Strukturfaktoren. Auch soll erwähnt werden, dass die mit st + bezeichneten Linien Flächen mit grösstem Strukturfaktor entsprechen. Einige grossen Differenzen sind vorhanden. So z. B. für die Reflexe 311 und 222, deren beobachtete Schwärzungsintensitäten

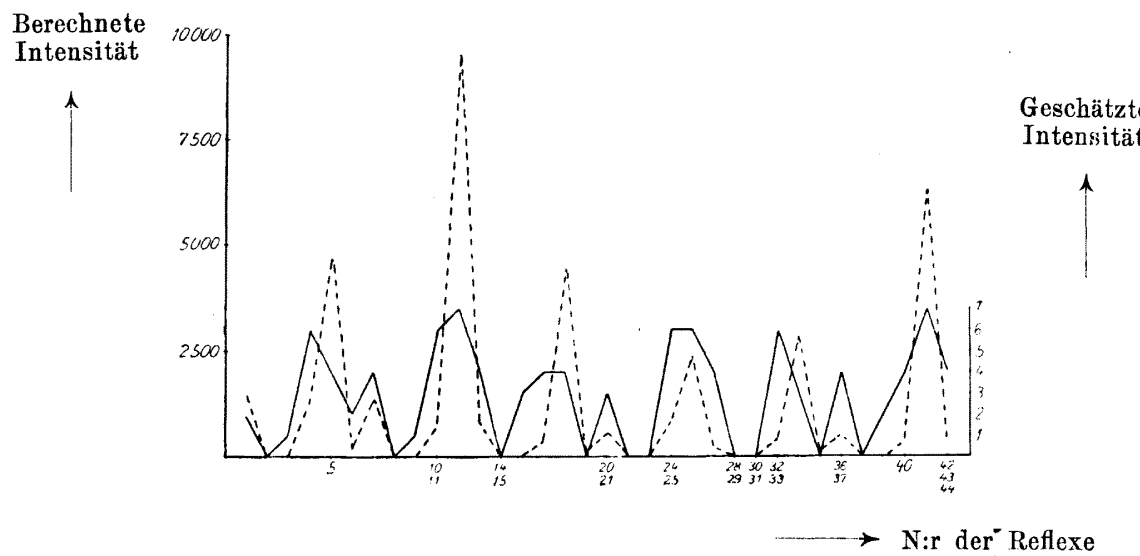

Fig. 9. Graphische Darstellung der Tab. 19. 
st und $\mathrm{m}$ sind, während dagegen die berechneten Werte 1257 bezw. 4800 werden.

In Struktur V wird jedes S-Atom in gleichem Abstand, nämlich $2.17 \AA$. E., von $3 \mathrm{Ni}$-A tomen und $1 \mathrm{Fe}$-Atom umgeben. $\mathrm{S}$ nimmt

Tab. 19. Vergleich zwischen den für die Pentlanditstruktur berechneten und geschätzten Intensitäten.

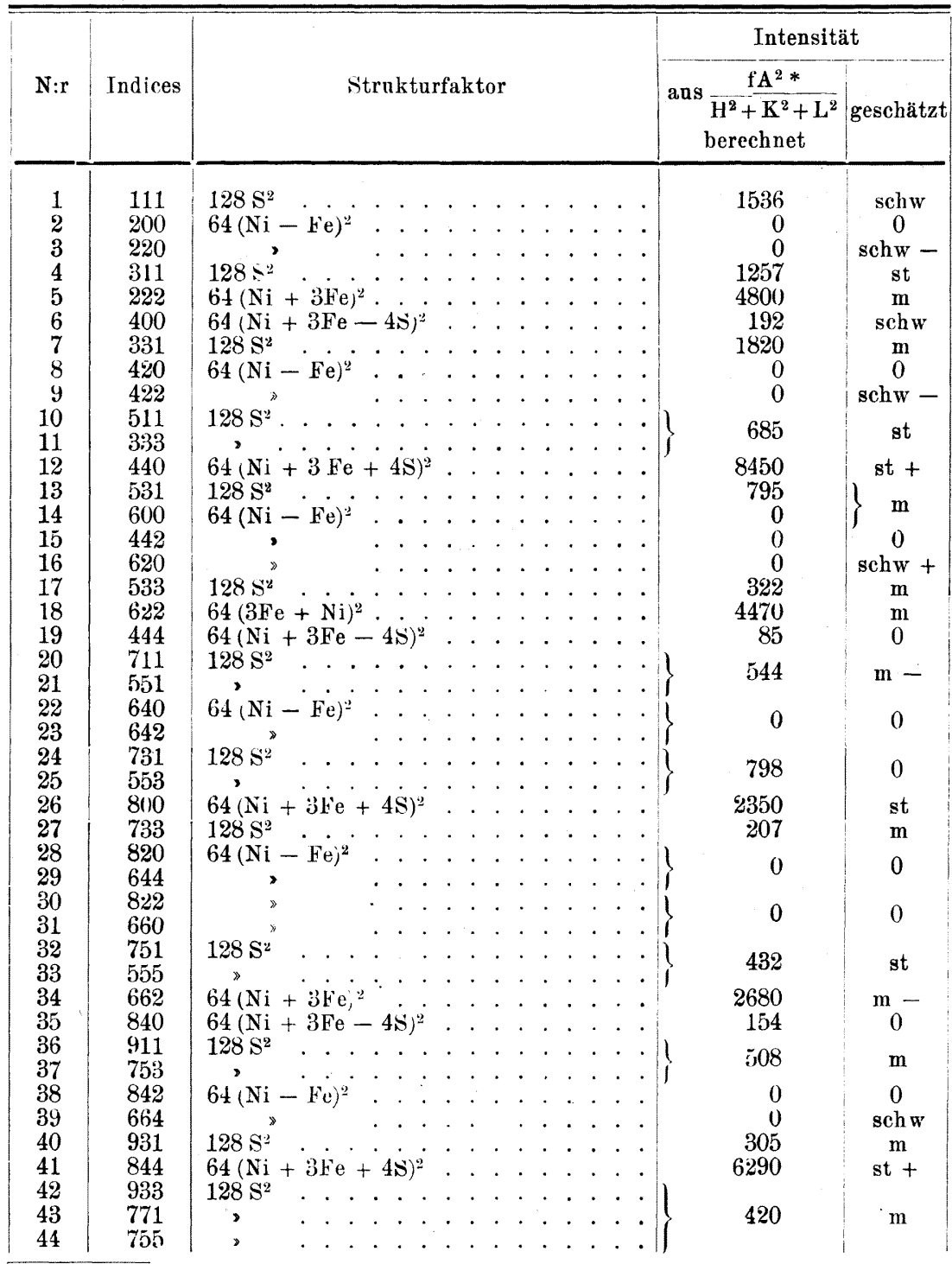

* $\mathrm{f}=$ Frequenzfaktor. $\mathrm{Fe}$ ist gleich $\mathrm{Ni}=4$ und $\mathrm{S}=3$ gesetzt. 
das Zentrum in einem kleinen Kubus mit der Kante $=\frac{1}{4}$ von der des Elementarkubus ein und mit $\mathrm{Ni}$ - und Fe-Atomen, die so in den Ecken placiert sind, wie Fig. 8 a zeigt. Jedes Fe-Atom ist von vier S-Atomen umgeben (Fig. 8 b) und jedes Ni-Atom ebenfalls von vier S-Atomen (Fig. 8 c).

Der Abstand $\mathrm{Fe}-\mathrm{Ni}=\frac{10.02 \cdot \sqrt{2}}{4}=3.54$ und $\mathrm{Fe}-\mathrm{Fe}$ bezw. $\mathrm{Ni}-$ $\mathrm{Ni}=\frac{1}{2} \cdot 10.02=5.01$. Die Atomplacierung der Struktur $\mathrm{V}$ scheint a priori annehmbar. Der Umstand, dass der Abstand Ni-S bezw. $\mathrm{Fe}-\mathrm{S}$ in dieser Struktur entschieden kürzer wird als in der Magnetkiesstruktur, deutet darauf, dass die gewählten Parameter nicht völlig korrekt sind. Mit etwas Anpassung derselben können wahrscheinlichere Werte für den Abstand zwischen den Atomen erhalten werden. $\mathrm{Ob}$ die berechneten Intensitäten durch eine solche Änderung der Parameterwerte durchschnittlich besser werden können, davon hat Verf. sich noch nicht überzeugt. Die Anomalien für die Reflexe 311 und 222 scheinen indessen zurückzubleiben.

\section{Millerit.}

Drehphotogramme. Ein kleiner Kristall von Millerit aus Wissen a. d. Sieg wurde mit der Längsrichtung des Kristalles parallel mit der Drehungsachse photographiert. Das erhaltene Photogramm enthält nur 0-Schicht und 1-Schicht. Der Abstand zwischen denselben beträgt $2.45 \mathrm{~cm}$. Hieraus erhält man $\mathrm{c}=3.14$ und die eine Konstante in der quadratischen Formel $\frac{L^{2}}{4 c^{2}}=0.0943$. Aus dem $\sin ^{2} \frac{\vartheta}{2}$ der 0 -Schicht erhält man mit Leichtigkeit die andere Konstante in der quadratischen Formel (Tab. 20) $\frac{\lambda^{2}}{3 \mathrm{a}^{2}}=0.0133$. Hieraus ergibt sich $a=9.66$. Das Achsenverhältnis wird 0.326, welcher Wert nahe dem von MrLLER ${ }^{1}$ morphologisch bestimmten 0.32955 liegt, und noch näher dem von Palache und $\mathrm{WOOD}^{2}$ erhaltenen 0.3274 .

Lauephotogramme. Nach mehreren Versuchen mit negativem Resultat ist es mir gelungen, mit Anwendung eines äusserst kleinen haarfeinen Kristalles von Wissen a. d. Sieg ein einheitliches und

1 Phil. Mag. 1835 6, 105.

2 Z. Kryst. XLI 1906, 1. 
Bd 47. H. 1.] UNTERSUCHUNG GEWISSER KRISTALLSTRUKTUREN.

Tab. 20. Drehphotogramm von Millerit, Drehachse || der Längsrichtung der Kristallnadeln.

\begin{tabular}{|c|c|c|c|c|}
\hline & $\begin{array}{l}\text { Indices Bravais, } \\
\text { dritter Index fort- } \\
\text { gelassen }\end{array}$ & $\begin{array}{l}\text { Schwär- } \\
\text { zung }\end{array}$ & gem. & $\sin ^{2} \frac{\theta}{2}$ \\
\hline 0 -Schicht & 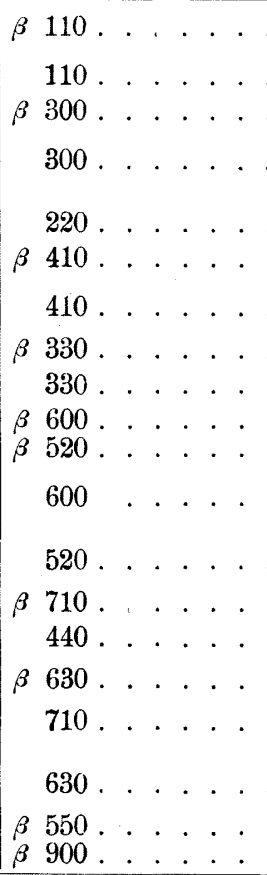 & $\begin{array}{l}\text { schw } \\
\text { m } \\
\text { schw } \\
\text { st } \\
\text { m } \\
\text { m } \\
\text { st } \\
\text { m } \\
\text { st } \\
\text { schw } \\
\text { schw } \\
\text { m } \\
\text { m } \\
\text { schw } \\
\text { st } \\
\text { schw } \\
\text { st } \\
\text { st } \\
\text { m- } \\
\text { m- }\end{array}$ & $\begin{array}{l}0.031 \\
0.039 \\
0.087 \\
0.119 \\
0.159 \\
0.232 \\
0.282 \\
0.299 \\
0.362 \\
0.398 \\
0.432 \\
0.485 \\
0.527 \\
0.635 \\
0.650 \\
0.704 \\
0.772 \\
0.856 \\
0.903 \\
0.942\end{array}$ & $\begin{array}{l}3 \cdot \frac{0.040}{3} \quad K_{1}=\frac{0.040}{3} \\
9 \cdot \frac{0.040}{3}=0.120 \\
12 \cdot \frac{0.040}{3}=0.160 \\
21 \cdot \frac{0.040}{3}=0.280 \\
27 \cdot \frac{0.040}{3}=0.360 \\
36 \cdot \frac{0.040}{3}=0.480 \\
39 \cdot \frac{0.040}{3}=0.520 \\
48 \cdot \frac{0.040}{3}=0.640 \\
57 \cdot \frac{0.040}{3}=0.760 \\
63 \cdot \frac{0.040}{3}=0.840\end{array}$ \\
\hline 1.Schicht & 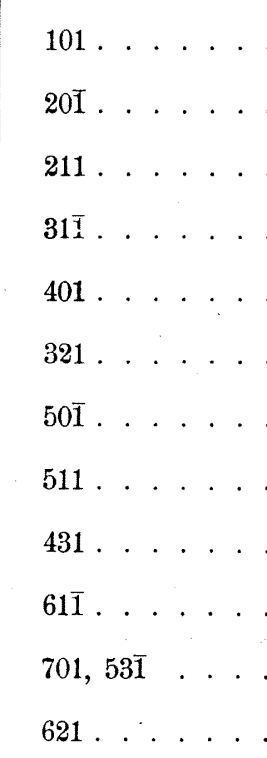 & $\begin{array}{c}\text { st } \\
\text { m } \\
\text { m } \\
\text { schw } \\
\text { schw } \\
\text { m } \\
\text { m + } \\
\text { st }\end{array}$ & $\begin{array}{l}0.107 \\
0.147 \\
0.185 \\
0.271 \\
0.315 \\
0.357 \\
0.438 \\
0.516 \\
0.591 \\
0.678 \\
0.751 \\
0.800\end{array}$ & $\begin{array}{l}\frac{0.040}{3}+0.094=0.107 K_{2}=0.094 \\
4 \cdot \frac{0.040}{3}+0.094=0.147 \\
7 \cdot \frac{0.040}{3}+0.094=0.187 \\
13 \cdot \frac{0.040}{3}+0.094=0.266 \\
16 \cdot \frac{0.040}{3}+0.094=0.307 \\
19 \cdot \frac{0.040}{3}+0.094=0.347 \\
25 \cdot \frac{0.040}{3}+0.094=0.424 \\
31 \cdot \frac{0.040}{3}+0.094=0.507 \\
37 \cdot \frac{0.040}{3}+0.094=0.587 \\
43 \cdot \frac{0.040}{3}+0.094=0.665 \\
49 \cdot \frac{0.040}{3}+0.094=0.747 \\
52 \cdot \frac{0.040}{3}+0.094=0.788\end{array}$ \\
\hline
\end{tabular}


deutliches, wenn auch schwaches Lauephotogramm (Fig. 10) zu erhalten. Die Strahlenrichtung war parallel mit der c-Achse und der Abstand Platte-Kristall = 32.6 $\mathrm{mm}$. Das Photogramm zeigt trigonale Symmetrie. Aus der gnom. Projektion des Lauephotogrammes ergibt sich das hexagonale Achsenverhältnis gleich 0.33.

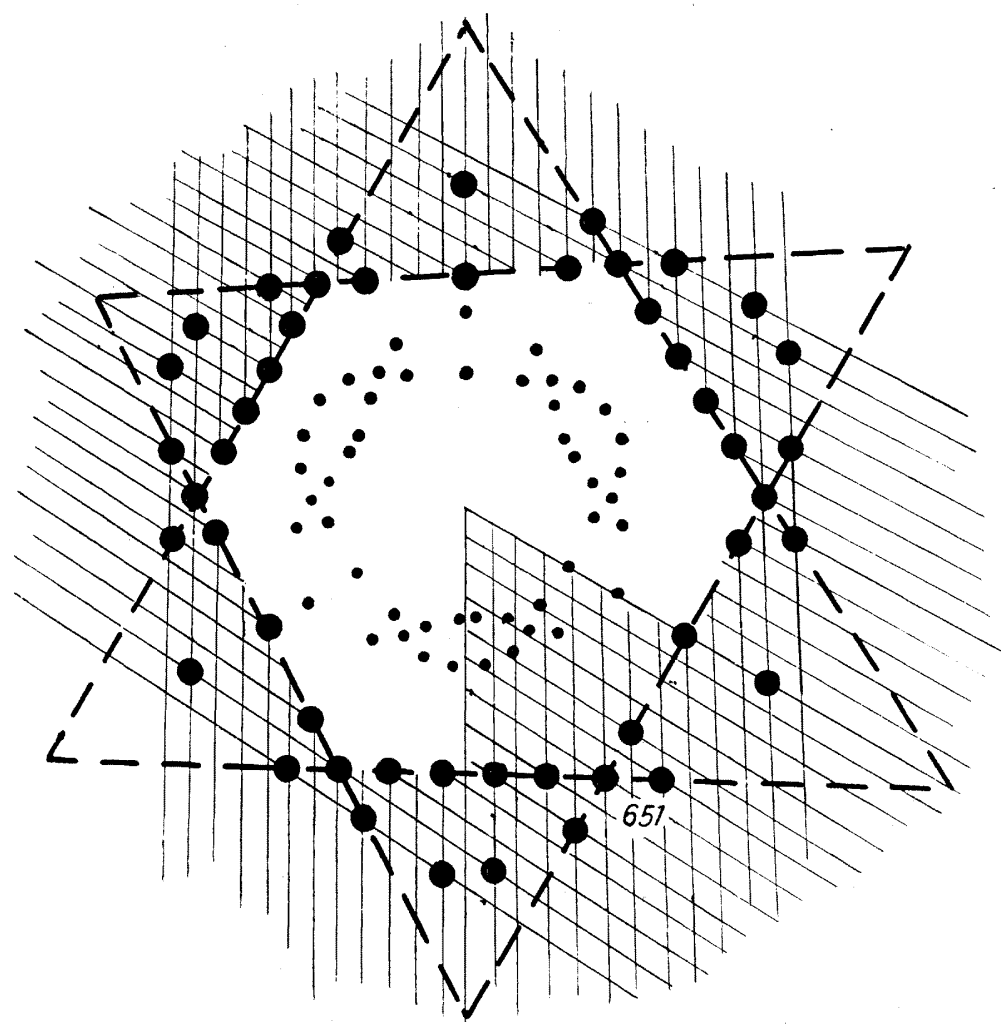

Fig. 10. Lanephotogramm senkrecht zur Basis und gnom. Projektion des Millerits. $(\mathrm{r}=1 \mathrm{~cm})$.

Durch Anwendung des Abstandes $11.5 \mathrm{~mm}$ vom Zentralfleck bis zum nächsten Reflexpunkt wird der kleinste Glanzwinkel $=9.7^{\circ}$. Dadurch kann ein approximativer Wert für die primitive Translation längs der c-Achse erhalten werden, nämlich $3.3 \AA$. E., wenn $\lambda$ min gleich 0.18 gesetzt wird. Dieser Wert ist approximativ gleich dem zuvor erhaltenen 3.14 .

Pulverphotogramme. Debyefilme sind von verschiedenen Kristallen vọn Wissen a. d. Sieg and von Müsen genommen worden, die 
Bd 47. H. 1.] [NTERSUCHUNG \%EWISSER KRISTALISTRUKTUREN.

letzteren sind als Beyrichit etikettiert. Die Photogramme (Tafel 3 und Tab. 21) sind im grossen ganzen identisch und bestätigen, dass die aus den Drehphotogrammen abgeleiteten Formelkonstanten wenigstens approximativ richtig sind. Die aus den Pulverphotogrammen, insbesondere dem Präzisionsphotogramm (Tafel 4 und Tab. 22) erhaltenen Konstanten sind in der hexagonalen Formel

Tab. 21. Pulverphotogramm von Millerit.

\begin{tabular}{|c|c|c|c|c|c|c|c|c|c|c|c|}
\hline \multirow{3}{*}{$\begin{array}{c}\text { Indices } \\
\text { Miller }\end{array}$} & \multicolumn{3}{|c|}{$\begin{array}{l}\text { a) Aus Mïsen, wahr- } \\
\text { scheinlich von Blei- } \\
\text { glanz und Linneit } \\
\text { verunreinigt }\end{array}$} & \multicolumn{2}{|c|}{$\begin{array}{l}\text { b) Aus Wissen } \\
\text { a. d. Sieg }\end{array}$} & \multirow{3}{*}{$\begin{array}{l}\text { In- } \\
\text { dices } \\
\text { Miller }\end{array}$} & \multicolumn{3}{|c|}{ Forts, von a) } & \multicolumn{2}{|c|}{ Forts. von b) } \\
\hline & \multirow{2}{*}{$\begin{array}{c}\text { Schwär- } \\
\text { zung }\end{array}$} & \multicolumn{2}{|c|}{$\operatorname{Sin}^{2} \frac{9}{2}$} & \multirow{2}{*}{$\begin{array}{l}\text { Schwär- } \\
\text { zung }\end{array}$} & \multirow[t]{2}{*}{$\operatorname{Sin}^{2} \frac{9}{2}$} & & \multirow{2}{*}{$\begin{array}{l}\text { Schwär- } \\
\text { zung }\end{array}$} & \multicolumn{2}{|c|}{$\operatorname{Sin}^{2} \frac{9}{2}$} & \multirow{2}{*}{$\begin{array}{c}\text { Schwär- } \\
\text { zung }\end{array}$} & \multirow{2}{*}{$\operatorname{Sin}^{2} \frac{9}{2}$} \\
\hline & & gem. & ber. & & & & & $\mathrm{em}$. & ber. & & \\
\hline & & & 0 & $w-$ & .032 & 422 & $\mathrm{~m}-$ & 488 & 0.488 & II & 0.48 \\
\hline & & & 0.041 & & .040 & $421 ?$ & $\mathrm{~m}-$ & 07 & 0.513 & & \\
\hline$\beta$ folg. & $\operatorname{sch} w$ & 0.066 & & & & 431 & $m+$ breit & 0.533 & 0.529 & $\operatorname{sch} w$ & 0.526 \\
\hline 2 & + & 0.078 & & & & 310 & $\mathrm{~m}$ & $0.550^{\circ}$ & 0.549 & $\mathrm{~m}$ & 0.549 \\
\hline$\beta 100$ & $\operatorname{sch} w+$ & 0.086 & 0.088 & & & $22 \%$ & $m+$ & $|0.595|$ & 0.590 & \} & \\
\hline & seh & 0. & 0.100 & $\operatorname{sch} w$ & 0.097 & 430 & W T & 0.590 & 0.594 & III & .09 \\
\hline 100 & $\mathrm{~m}+$ & 0.106 & 0.107 & $\operatorname{sch} w$ & 0.105 & $\beta 420$ & & & 0.619 & & \\
\hline$\beta 111$ & st + & 0.121 & 0.121 & 8 & 0.118 & $\beta \quad 441$ & $\operatorname{sch} w$ & 0.628 & 0.623 & $\int \operatorname{seh} w-$ & 0.623 \\
\hline 211 & $\int \operatorname{sch} w$ & 0.132 & 0.122 & $\operatorname{seh} w-$ & 0.130 & $\begin{array}{r}321 \\
\beta 532\end{array}$ & $\operatorname{sch} w+$ & 638 & $\begin{array}{l}0.630 \\
0.637\end{array}$ & $\int \operatorname{sch} w-$ & 632 \\
\hline 111 & $m+$ & 6 & 0.147 & $\mathrm{~m}$ & 0.145 & 440 & $\operatorname{seh} w+$ & 0.652 & 0.651 & $\mathrm{~m}$ & 0.645 \\
\hline$\beta 210$ & & & 0.155 & & & 432 & $\operatorname{sch} w+$ & 0.679 & 0.675 & $\mathrm{~m}$ & 0.672 \\
\hline $20 \%$ & & & 0.162 & $\operatorname{sch} w+$ & 0.162 & 411 & $\operatorname{seh} v$ & & 0.711 & & \\
\hline$?(\beta)$ & sc & 0.1 & & & & $\begin{array}{ll}\beta & 431 \\
\beta & 210\end{array}$ & breit & 0.719 & 0.720 & $\mathrm{~m}$ & 0.715 \\
\hline $\begin{array}{l}210 \\
?\end{array}$ & & & 0.188 & $\mathrm{~m}$ & 0.185 & $\beta \frac{210}{420}$ & & & $\begin{array}{l}0 . \\
0 .\end{array}$ & r & \\
\hline$\beta 221$ & & & 0.222 & $\operatorname{sch} w$ & 0.218 & 522 & st + & 0.760 & 0.756 & st + & 0.751 \\
\hline$\beta 321$ & & 0.2 & 0.229 & $\operatorname{sch} w-$ & 0.234 & $4 \overline{4} 1$ & & & 0.756 & & \\
\hline & & & & & & 532 & $\mathrm{~m}$ & 0.773 & 0.773 & st - & 0.767 \\
\hline$\beta \overline{311}$ & & & 0.255 & & & 332 & $\mathrm{~m}-$ & 0798 & 0.793 & t) & 0.79 \\
\hline $2 \overline{2} 1$ & & 0. & 0.269 & st & 0.265 & 531 & breit & 0.180 & 0.797 & & 0.10 \\
\hline & & & 0.284 & st - & 0.281 & 111 & & & 0.839 & & \\
\hline$\beta 320$ & & & 0.288 & & & 54 & $\mathrm{~m}-$ & 0.854 & 0.853 & $\mathrm{~m}$ & 0.846 \\
\hline$\beta \underline{303}$ & & & 0.302 & & & 431 & st & 0.880 & 0.874 & ? & 0.872 \\
\hline & & 0 & 0 & st - & 0.309 & 210 & & & 0 & & \\
\hline$\beta 110$ & & & 0318 & & & & $\operatorname{sch} w-$ & 0.890 & & $\operatorname{sch} w$ & 0.883 \\
\hline $3 \overline{2}$ & $\mathrm{~m}$ & & 0.350 & $\mathrm{~m}$ & 0.346 & 540 & st & 0.922 & 0.918 & st & 0.91 \\
\hline $30 \overline{3}$ & & 0. & 0 & st & 0.363 & & $\operatorname{sch} w+$ & $\mid 0.934$ & & & \\
\hline 110 & $\mathrm{~m}$ & 0.391 & 0.387 & $\mathrm{~m}$ & 0.386 & 521 & & & 0.955 & & \\
\hline 200 & & & 0.427 & & & 533 & st & 0.959 & 0.959 & st & 0.95 \\
\hline $\begin{array}{l}322 \\
3431\end{array}$ & $\operatorname{sch}$ & 0.426 & $\begin{array}{l}0.432 \\
0.436\end{array}$ & $\operatorname{seh} w-$ & 0.42 & $\begin{array}{l}300 \\
221\end{array}$ & & & $\begin{array}{c}0.961 \\
,\end{array}$ & & \\
\hline$a$ & & & 0.468 & $\operatorname{sen}$ & 0.46 & & $\mathrm{~m}$ & 0.971 & & & \\
\hline & & & & & & & & & & & \\
\hline
\end{tabular}

Formelkonstanten: $\mathrm{Fe}_{K}\left\{\begin{array}{l}0.1068 \\ 0.1730\end{array}\right.$ - $\mathrm{e}_{K}\left\{\begin{array}{l}0.0880 \\ 0.1426\end{array}\right.$

$$
\text { Formel: } \sin ^{2} \frac{\vartheta}{2}=\mathrm{C}_{1}\left(\mathrm{H}^{2}+\mathrm{K}^{2}+\mathrm{I}^{2}\right)+\mathrm{C}_{2}\left(\mathrm{HK}+\mathrm{HL}+\mathrm{KL}_{4}\right)
$$

5-250333. G.. F. F. 1925. 
$\frac{\lambda^{2}}{3 \mathrm{a}^{2}}=0.01350$ und $\frac{\lambda^{2}}{4 \mathrm{c}^{2}}=0.0933 . \quad$ Hierans $\mathrm{a}=9.60 \mathrm{c}=3.15 \quad$ und $\frac{c}{a}=0.328$.

Die hexagonalen Indices der beobachteten Reflexe erfüllen sämtlich die Rhomboederbedingung, d. h.: $2 \mathrm{H}+\mathrm{K}+\mathrm{L}=3 \mathrm{n}$ (n eine ganze Zahl). Dies lässt auf einen rhomboedrischen Primitivparallelepiped schliessen; in den Tabellen sind auch rbomboedrische Indices gewählt. Die Kante des Rhomboeders $\mathrm{r}$ wird 5.64 und der Polkantenwinkel $\mathrm{v}=116.6$. Hier ist $\mathrm{zu}$ bemerken, wie ungewöhnlich flach das Elementarrhomboeder ist.

Anzahl Moleküle im Elementarrhomboeder. Für das Volumen des Elem.-rhomboeders erhält man zwei Ausdrücke, woraus die Anzahl Moleküle in demselben auf 3.2 berechnet wird, wenn man das spez. Gewicht gleich 5.5 setzt. Geht man davon aus, dass die Anzahl 3 ist, so erhält man das spezifische Gewicht $=5.31$; hiermit wird das von Miluer angegebene 5.26-5.30 und von Hintze »bis 5.9 selten unter 5.3 » verglichen.

Tab. g2. Präzisionsphotogramm von Millerit ans Wissen a. d. Sieg.

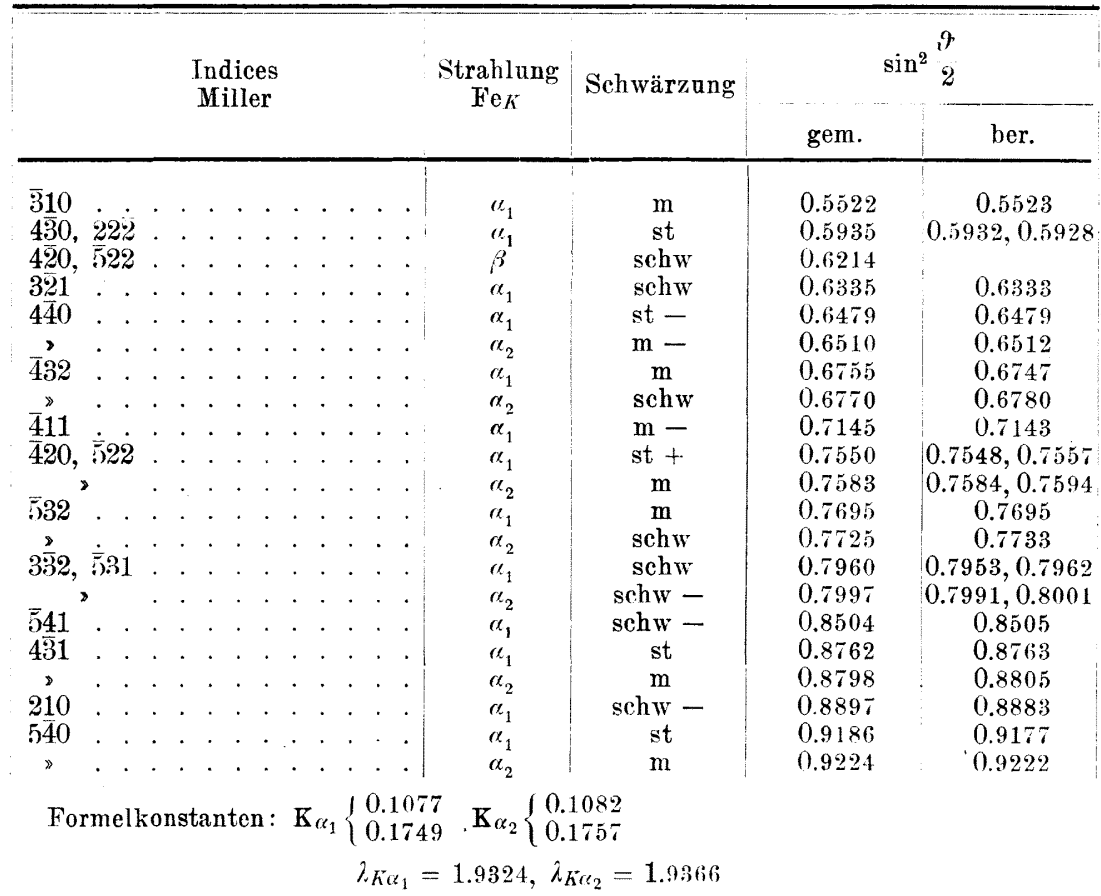


Strukturbestimmung. Drei Moleküle NiS sollen in einem rhomboedrischen Elementarparallelepiped placiert werden. Innerhalb des rhomboedrischen Systemes haben nur folgende Raungruppen dreizählige Lagen, nämlich bei Voraussetzung rhomboedrischer Achsen

$$
\begin{aligned}
& \mathbf{C}_{3}^{4} \mathbf{m n p}, \mathrm{pmn}, \mathrm{npm} \\
& \mathbf{C}_{3 \mathrm{i}}^{2}=\mathbf{D}_{3 \mathrm{~d}}^{5} 1 / 200,0^{1 / 20}, 00^{1 / 2} ;{ }^{1 / 2} / 20,0^{1 / 2^{1 / 2}}, 1^{1 / 2} 0^{1 / 2} \\
& \mathbf{C}_{3 \mathrm{v}}^{5} \mathrm{mmp}, \mathrm{pmm}, \mathrm{mpm} \\
& \mathbf{D}_{3}^{7} 0 p \bar{p}, \bar{p} 0 p, p \bar{p} 0 ;{ }^{1 / 2} p \bar{p}, \bar{p}^{1 / 2 p}, p \bar{p}^{1 / 2} \text {. }
\end{aligned}
$$

Die erste Raumgruppe ist als Untergruppe in allen übrigen vorhanden, daher kann eine für alle gültige Diskussion bei jener durchgetührt werden.

Wie aus den beiliegenden Photogrammen von Millerit (Tafel 3 und 4) ersichtlich, sind dieselben ausserordentlich linienreich, und Tab. 20 und 21 zeigen, dass es Linien mit so gut wie allen denkbaren Indices enthält. Doch fehlt auf den Photogrammen der Reflex 111 (Bravais 003), was bedeutet, dass approximativ gleich beugende Ebenen in einem Abstand von einander annäherungsweise $\frac{\mathrm{c}}{6}$ längs der c-Achse gerechnet, vorhanden sein müssen.

Geht man von der allgemeinen dreizähligen Lage, vertreten durch die Koordinaten mnp, pmn, npm und rst, trs, str, aus, so findet man, dass die Atome um die c-Achse als Trigyre geordnet sind. Für die Fläche 111 erhält man den Strukturfaktor $\mathrm{A}^{2}=9\left[\mathrm{Ni}^{2}+\mathrm{S}^{2}+\right.$ $2 \mathrm{NiS} \cos 2 \pi(\mathrm{m}+\mathrm{n}+\mathrm{p}-\mathrm{r}-\mathrm{s}-\mathrm{t})]$.

Damit $A^{2}$ klein werden soll, ist erforderlich, dass der cos-Faktor so nahe -1 wie möglich kommt. In solchem Fall muss $(m+n+p$ $-\mathrm{r}-\mathrm{s}-\mathrm{t}) \sim \frac{1}{2}, \frac{3}{2} \mathrm{u}$. s. w. sein. Eine dreizählige S-Gruppe muss demnach annäherungsweise mitten zwischen zwei dreizähligen $\mathrm{Ni}$ Gruppen liegen. Hierdurch wird der Abstand zwischen einer Ni-Gruppe und der nächsten S-Gruppe, längs der c-Achse gerechnet, gleich $\frac{\mathrm{c}}{6}$. $\mathrm{A}^{2}$ erhält den approximativen Wert $9(\mathrm{Ni}-\mathrm{S})^{2}$. In der vorigen NiS-Struktur fehlen Reflexe, wenn in den $\mathrm{A}^{2}$-Werten der Faktor (Ni - S) darin steckt. Man kann da behaupten, dass das auch hier der Fall sein wird.

Durch graphische Darstellung der Strukturfaktoren für gewisse Retlexe, die entweder ausgeprägt stark oder schwach sind, hat Verf. versucht, die Parameterwerte zu bestimmen. Nur in Ver- 
bindung mit Versuchen an Strukturmodellen hat diese Methode zu einem Resultat geführt.

Wenn man mit Wachskugeln als Atome die Struktur so dicht wie möglich aufbaut, ohne dass die oben gemachten Voraussetzungen wegfallen, erhält man als wahrscheinliche Atomgruppierung für Ni: to0, 0t0, oot und für $S$ : ppq, qpp, pqp (Fig. 11).

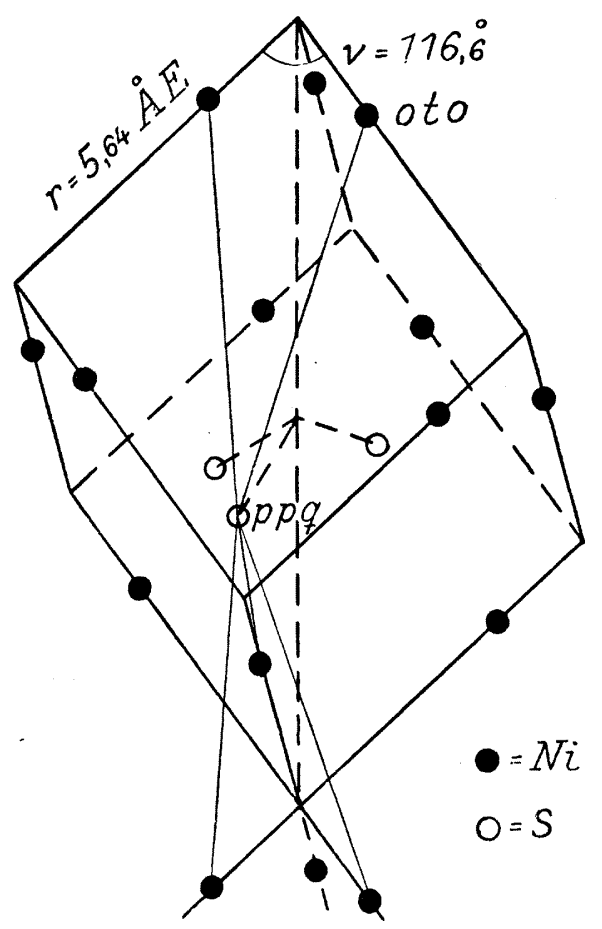

Fig. 11. Struktur des Millerits.

Hierdurch wird die Anzahl der Parameter von 6 auf 3 vermindert. Dann kann die vorstehend gegebene Bedingung für die Atomkoordinaten auf $2 p+q=t+\frac{3}{2}$, vereinfacht werden, wodurch sich $p$ mit bekannten Werten von $t$ und $q$ berechnen lässt.

$\mathrm{Um} t$ und $\mathrm{q} z u$ bestimmen ist Verf. von der Annahme ausgegangen, dass ein Ni-Attom benachbarte S-Atome in gleichem Abstand um sich gruppiert, nämlich ähnlich dem Abstand, der in dem andern Strukturtyp erhalten worden ist. Zwei Fälle sind möglich. 
Bd 47. H. 1.] UNTERSUChUNG GEWISSER KRISTALLSTRUKTUREN.

In fig. 12 ist der Abstand von $\mathrm{C}$ zum Centrum der Figur = $(1-t) \frac{a}{\sqrt{3}}$ und von $B=\frac{y \cdot a}{\sqrt{3}}$, wenn $q-p=y$ gesetzt wird. Also wird $\mathrm{BC}^{2}=\left[(1-t)^{2}+\mathrm{y}^{2}-\mathrm{y}(1-\mathrm{t})\right]^{2} \cdot \frac{\mathrm{a}^{2}}{3}+\frac{\mathrm{c}^{2}}{36}$ und $\mathrm{AB}^{2}=(\mathrm{t}-\mathrm{y})^{2}$. $\frac{\mathrm{a}^{2}}{3}+\frac{\mathrm{c}^{2}}{4} . \quad$ Für $\mathrm{BC}=\mathrm{AB}$ erhält man $\mathrm{y}=\frac{0.93-2 \mathrm{t}}{1-3 \mathrm{t}}$. Weiter hat $\operatorname{man} \mathrm{t}+1.5=2 \mathrm{p}+\mathrm{q}$ und $\mathrm{q}-\mathrm{p}=\mathrm{y}$.

Wird nun $t=0.53$ gewählt, so wird $q=0.82$ und $p=0.60$ und der Abstand $\mathrm{AB}=\mathrm{BC}=2.3$.
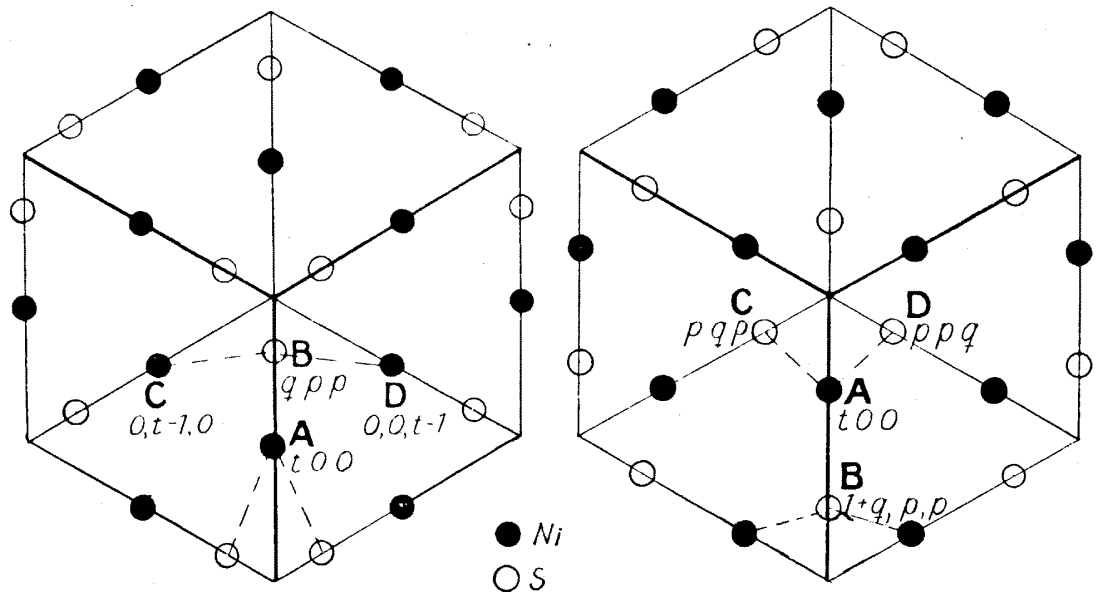

Projcktion auf Basis von den Atomen der vorgehenden Figur für versehicdene Werte der Parameter.

Fig. 12.

$\mathrm{C}$ und $\mathrm{D} \frac{\mathrm{c}}{6}$ über $\mathrm{B}$

A $\frac{c}{2}$ sowohl ïber als unter $B$.
Fig. 13.

$\mathrm{C}$ and $\mathrm{D} \frac{\mathrm{C}}{2}$ sowohl über als unter $\mathrm{A}$

B $\frac{\mathrm{c}}{6}$ unter A.

Diese Parameterwerte geben indessen keine gate Übereinstimmung zwischen beob. und ber. Intensitäten. Ebenso wenig ist es gelungen, durch kleinere Verschiebungen der Parameterwerte die Übereinstimmung befriedigend zu erhalten.

2) Aus Fig. 13 erhält man:

$$
(1-y-t)^{2} \cdot\left(\frac{a}{\sqrt{3}}\right)^{2}+\left(\frac{c}{b}\right)^{2}=\left(t^{2}+y^{2}-t y\right) \cdot\left(\frac{a}{\sqrt{3}}\right)^{2}+\left(\frac{c}{2}\right)^{2}
$$

Hieraus $\mathrm{y}=\frac{0.93-2 \mathrm{t}}{2-3 \mathrm{t}} \cdot$ Wählt man nun $\mathrm{t}=\frac{1}{3}$, so wird $\mathrm{y}=0.26$.

Für die Bestimmung der Parameter $p$ und $q$ erhält man 


$$
\left.\begin{array}{rl}
2 p+q=1.83 \\
p-q=y=0.26
\end{array}\right\} \begin{aligned}
& p=0.70 \\
& q=0.43
\end{aligned}
$$

Der Abstand $A C$ wird $=A B=2.3$.

Die Übereinstimmung zwischen beob. und ber. Intensitäten (Fig. 14 und Tab. 23) dürfte als befriedigend anzusehen sein. Möglicherweise könnten kleinere Änderungen der Parameterwerte die Übereinstimmung noch grösser machen.

Schliesslich muss auch hier hervorgehoben werden, dass beim Glühen in $\mathrm{H}_{2} \mathrm{~S}$-Atmosphäre eine Umlagerung der A tome stattfindet, und dass die neue Substanz dieselbe Struktur wie Magnetkies hat, wie aus Tab. 11 ersichtlich.

Von grossem Interesse wäre es natürlich den Zusammenhang

Tab. 23. Vergleich zwischen den fïr die Milleritstruktur berechneten und geschätzten Intensitäten.

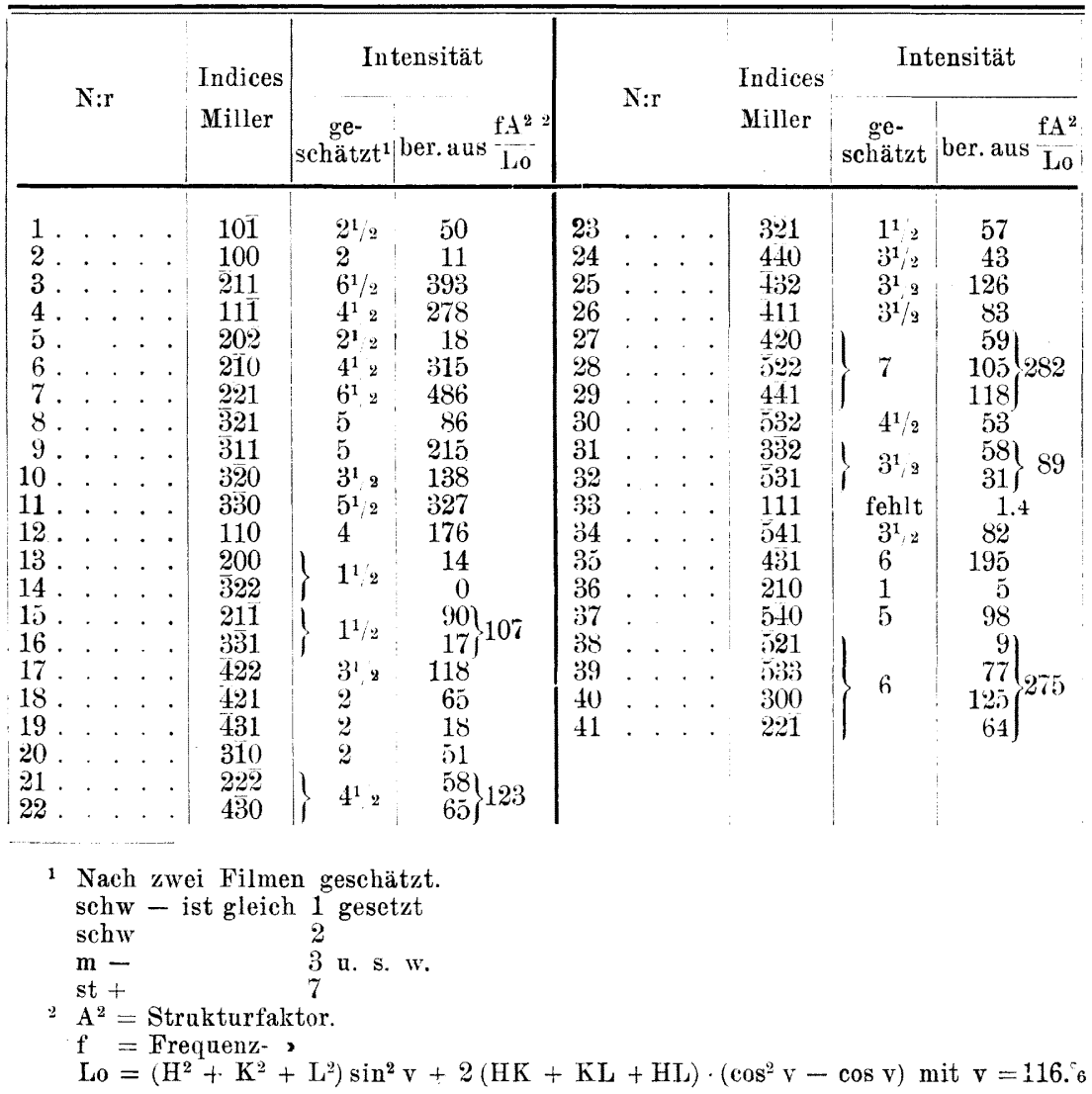


zwischen der von Palache und Wood ${ }^{1}$ bei Millerit nachgewiesenen Zwillingbildung durch Gleiten und der vorstehend abgeleiteten Struktur zu untersuchen. $\mathrm{Zu}$ einer solchen Úntersuchung ist es indessen noch nicht gekommen.

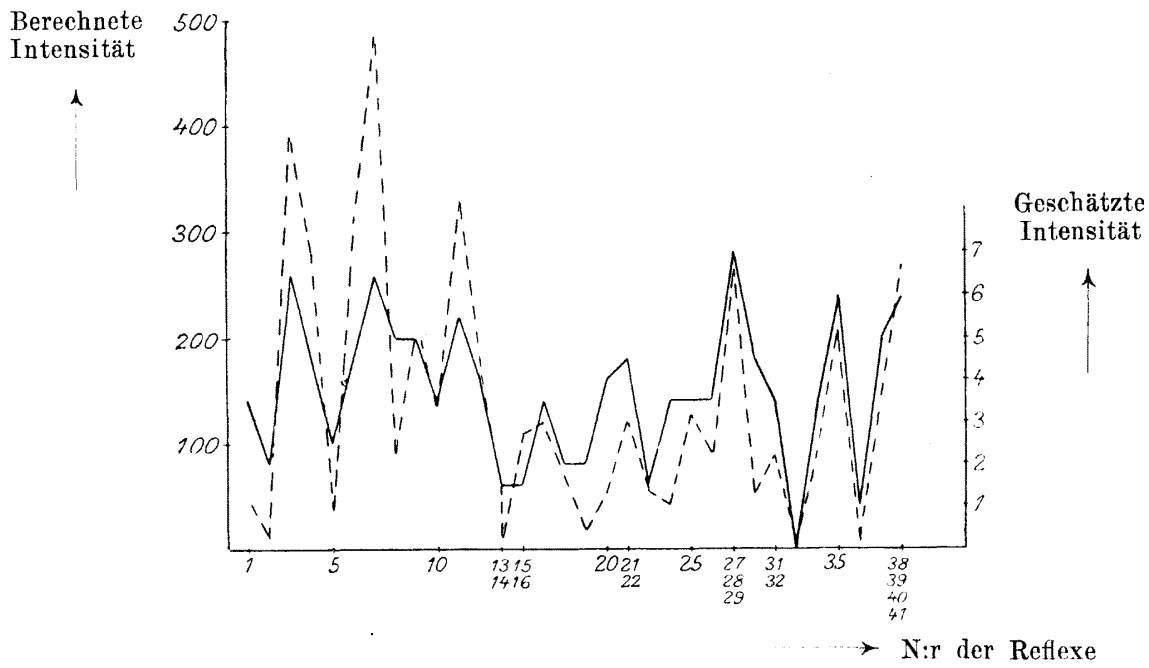

Fig. 14. Graphische Darstellnng der Tah. 23.

\section{Zusammenfassung.}

1) Die Kristallstrukturen von Magnetkies (Troilit) und Breithauptit sind von demselben Typus wie die des Rotnickelkieses. Raumgruppe $D_{6 \mathrm{~h}}^{4}$. Atomkoordinaten $\left\{\begin{array}{l}000,00 \frac{1}{2} \\ \frac{1}{3} \frac{2}{3} \frac{1}{4}, \frac{2}{3} \frac{1}{3} \frac{3}{4}\end{array}\right.$

2) Die künstlich dargestellten Verbindungen FeSe, NiS, NiSe, $\mathrm{CoS}$ und deren Mischkristalle realisieren gleichfalls denselben Strukturtyp.

3) Magnetkies ist wahrscheinlich eine feste Lösung von $\mathrm{S}$ in $\mathrm{FeS}$, in welcher S-Atome Fe-Atome substituieren.

4) Die Achsenverhältnisse der obengenannten Verbindungen schwanken zwischen den Werten 1.3 bis 1.7.

5) Wenn ein Mischkristall (Ni, Fe)S geschmolzen wird, entsteht bei der Erstarrung Pentlandit. Und umgekehrt lässt sich Pentlandit durch Glühen in $\mathrm{H}_{2} \mathrm{~S}$-Gas in Mischkristall verwandeln.

6) Der Elementarkubus des Pentlandits hat die Kante gleich 10.0 A. E. und enthält 32 S-A tome und gleich viele Metall-Atome 
mit $\mathrm{Ni}$ und $\mathrm{Fe}$ in wechselnder Menge. Die wahrscheinlichste Struktur gehört der Raumgruppe $\mathrm{O}_{\mathrm{h}}^{5}$.

7) Die für Millerit gefundene Kristallstruktur ist vor allem durch das sehr flache Elementarrhomboeder charakterisiert. Die Kante ist 5.64 A. E. und der Polkantenwinkel 116.6. Drei Moleküle sind im Elementarbereich vorhanden. Die Struktur gehört der Raumgruppe $\mathrm{C}_{3 \mathrm{v}}^{5}$, und jedes Atom ist von 5 anderen in gleichem Abstand umgeben.

Wenn man Millerit in $\mathrm{H}_{2} \mathrm{~S}-$ Gas glüht, erhält man NiS mit der Magnetkies-Struktur.

Stockholms Högskolas Mineralogiska Institut, Februari 1925. 


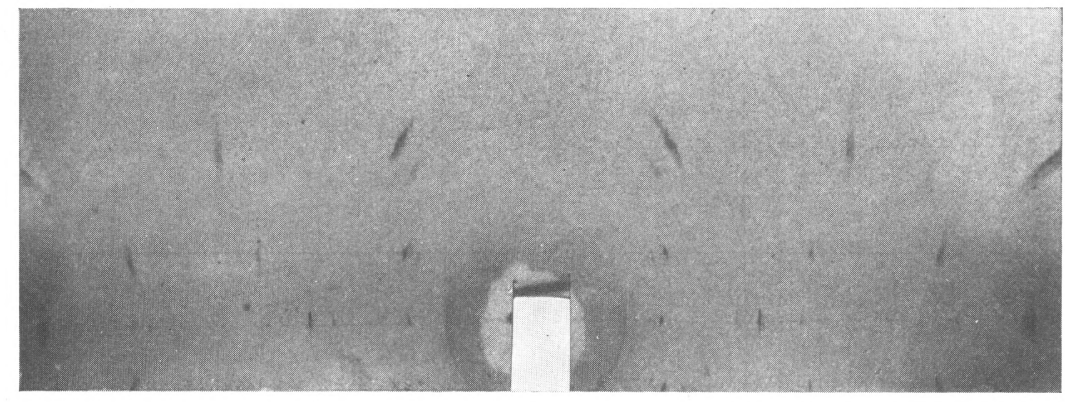

Drehphotogramm von Magnetkies. Drehachse senkrecht zur Basis.

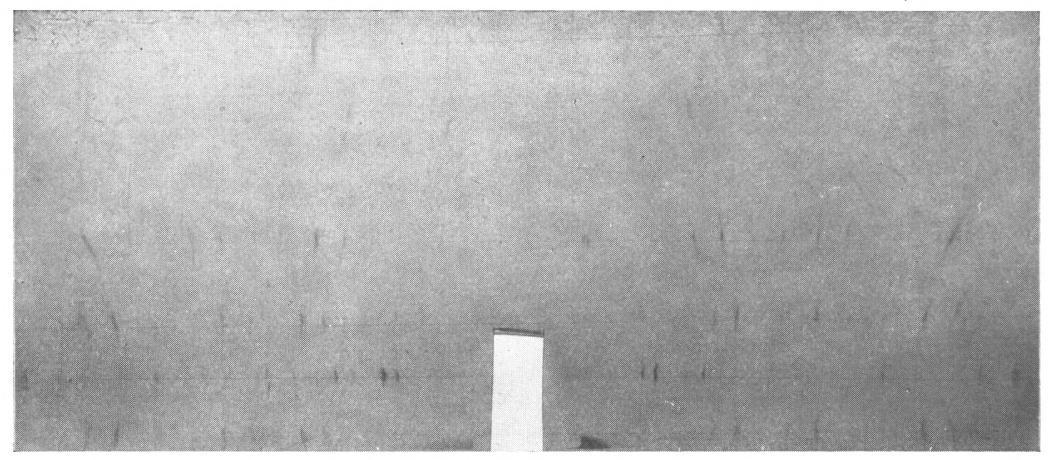

Drehphotogramm von Covellin. Drehachse eine Basiskante.

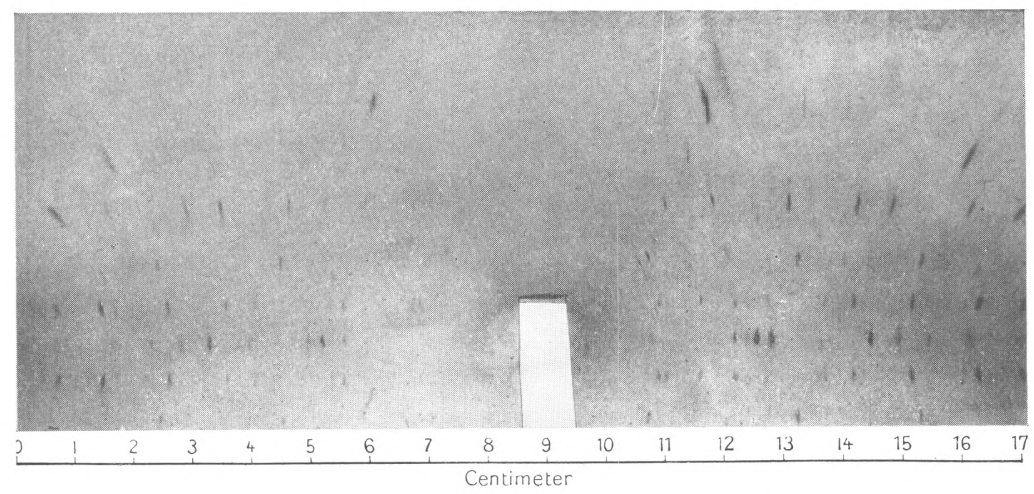

Drehphotogramm von Pentlandit. Drehachse eine Kubuskante 


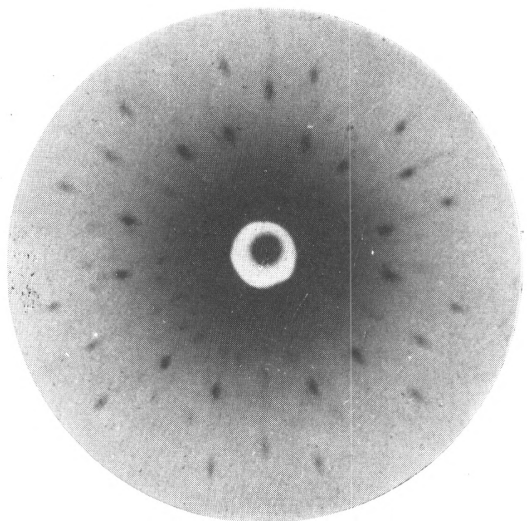

Lauephotogramm von Magnetkies senkrecht zur Basis.

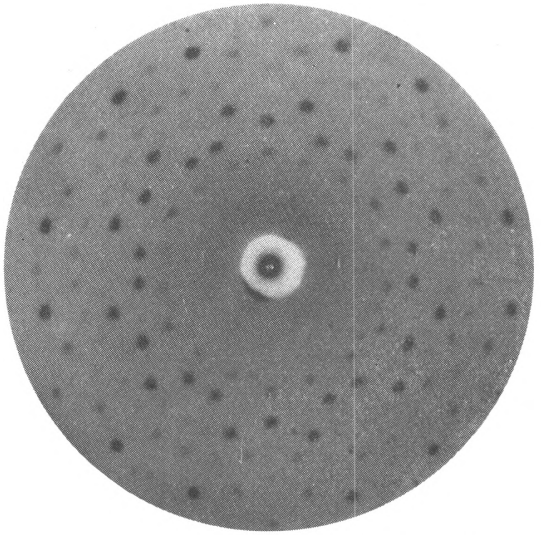

Lauephotogramm von Breithauptit senkrecht zur Basis.

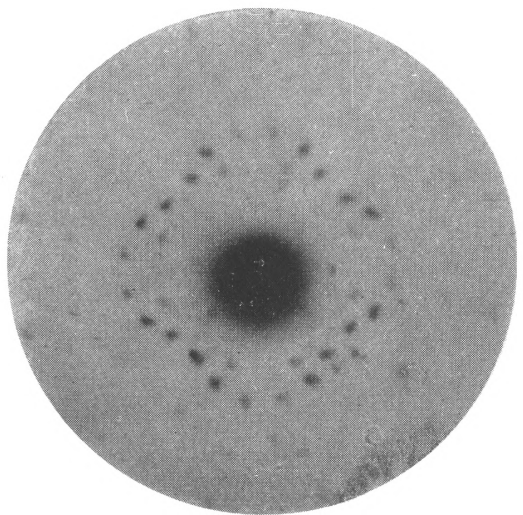

Lauephotogramm von Pentlandit senkrecht zu einer Kubusfläche. 


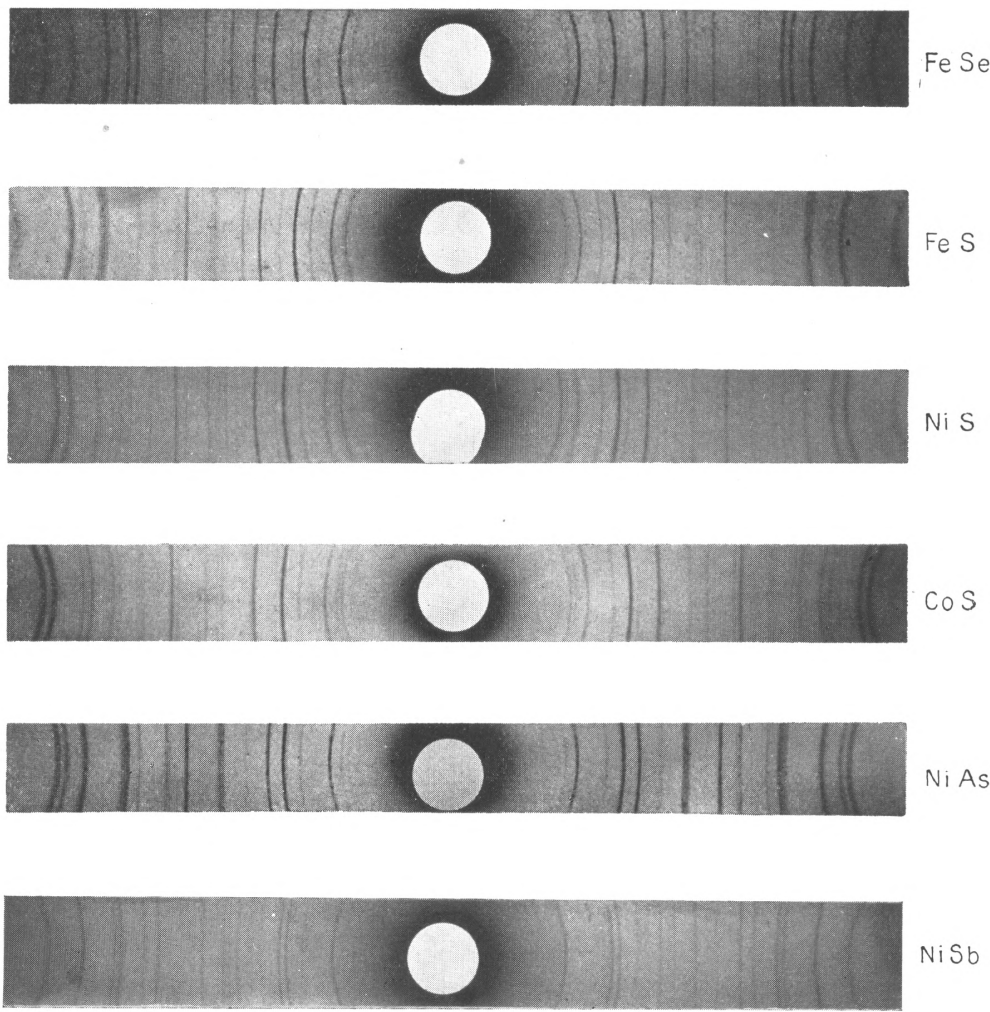

$\mathrm{NiSb}$

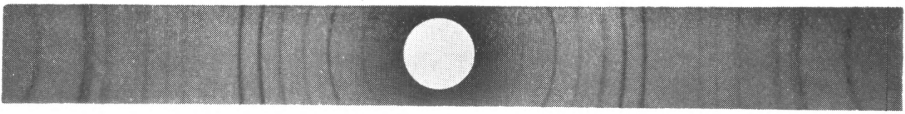

$\mathrm{Ni}_{3} \mathrm{~S}_{2}$

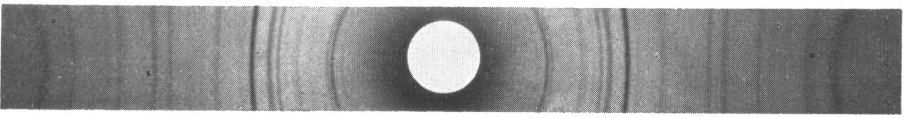

Pent. landit

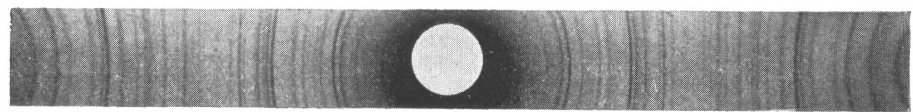

Mil-

lerit

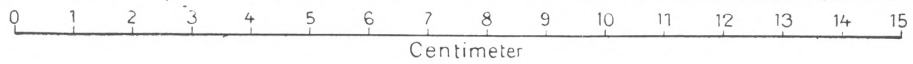

Pulverphotogramme. 



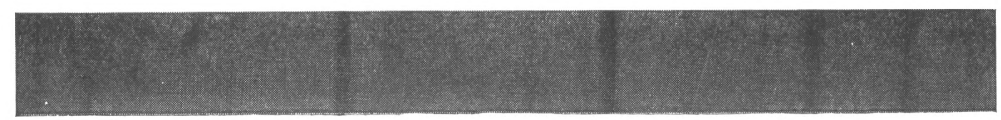

'Präzisionsphotogramm von Millerit.

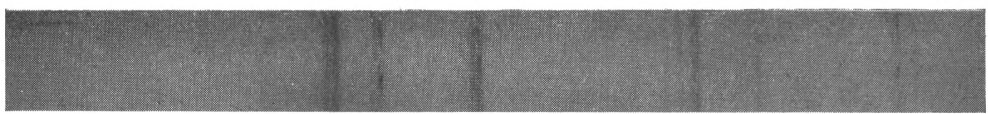

Präzisionsphotogramm von Rotnickelkies.

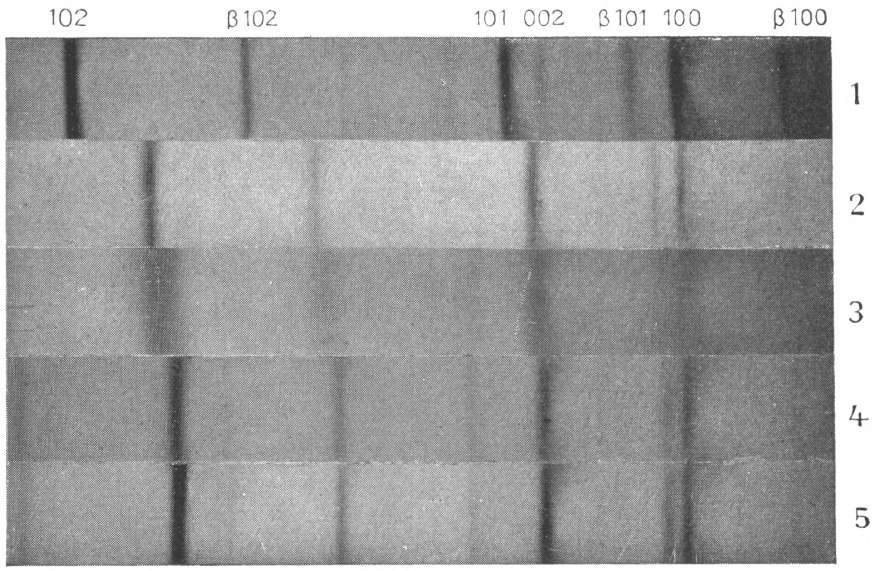

Präzisionsphotogramm von NiS und FeS.

1. Nis.

2. FeS mit Schwefelïberschuss.

3. Troilit, Gibeon.
4. Troilit, Augustinowka.

5. Tellurischer Troilit.
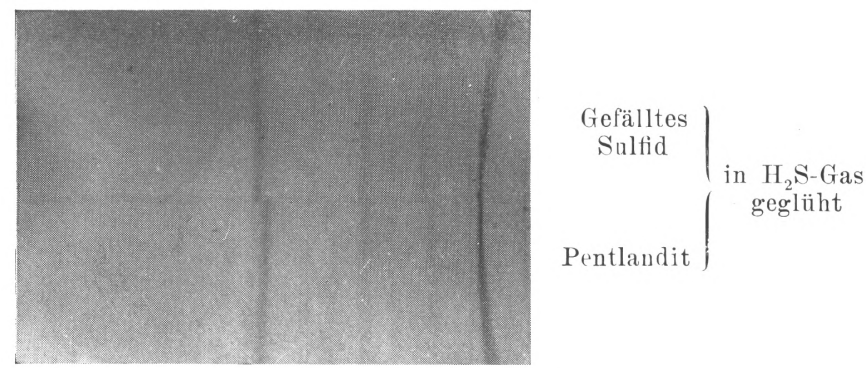

Präzisionsphotogramm von (Ni, Fe)S. 

Stockholm 1925. P. A. Norstedt \& Söner. 250333 